\title{
NUMERICAL METHODS OF COMPUTATION OF SINGULAR AND HYPERSINGULAR INTEGRALS
}

\author{
I. V. BOIKOV
}

(Received 21 September 2000 and in revised form 5 August 2001)

\begin{abstract}
In solving numerous problems in mathematics, mechanics, physics, and technology one is faced with necessity of calculating different singular integrals.

In analytical form calculation of singular integrals is possible only in unusual cases. Therefore approximate methods of singular integrals calculation are an active developing direction of computing in mathematics. This review is devoted to the optimal with respect to accuracy algorithms of the calculation of singular integrals with fixed singularity, Cauchy and Hilbert kernels, polysingular and many-dimensional singular integrals. The isolated section is devoted to the optimal with respect to accuracy algorithms of the calculation of the hypersingular integrals.
\end{abstract}

2000 Mathematics Subject Classification. 65D32.

\section{Introduction}

1.1. Definitions of optimality. The developing of optimal methods for solving problems of computational mathematics is of prime importance. Various definitions of optimality of numerical methods, basic results on optimal algorithms and a detailed bibliography can be found in [1, 3, 47]. Recall definitions of the algorithms, optimal with respect to accuracy, for calculation of singular integrals. We use the definitions from [3] of algorithms, optimal with respect to accuracy. The definitions of optimal with respect to accuracy algorithms are different for singular integrals with fixed and with moving singularities.

Consider a quadrature rule

$$
\int_{-1}^{1} \frac{\phi(\tau)}{\tau} d \tau=\sum_{k=1}^{N} p_{k} \phi\left(t_{k}\right)+R_{N}\left(\phi, p_{k}, t_{k}\right),
$$

where coefficients $p_{k}$ and nodes $t_{k}, k=1, \ldots, N$, are arbitrary.

An error of the quadrature rule (1.1) on class $\Psi$ is defined as

$$
R_{N}\left(\Psi, p_{k}, t_{k}\right)=\sup _{\phi \in \Psi}\left|R_{N}\left(\phi, p_{k}, t_{k}\right)\right|
$$

Define a functional $\zeta_{N}[\Psi]=\inf _{p_{k}, t_{k}} R_{N}\left(\Psi, p_{k}, t_{k}\right)$.

The quadrature rule with coefficients $p_{k}^{*}$ and nodes $t_{k}^{*}$ is optimal, asymptotically optimal, optimal with respect to order on the class $\Psi$ among all quadrature rules of type (1.1) provided that $R_{N}\left(\Psi, p_{k}^{*}, t_{k}^{*}\right) / \zeta_{N}[\Psi]=1, \sim 1, \asymp 1$. 
Define optimality with respect to accuracy for singular integrals with moving singularity. Consider a quadrature rule

$$
\frac{1}{2 \pi} \int_{0}^{2 \pi} \phi(\sigma) \operatorname{ctg} \frac{\sigma-s}{2} d \sigma=\sum_{k=1}^{N} p_{k}(s) \phi\left(t_{k}\right)+R_{N}\left(s, \phi, p_{k}, t_{k}\right) .
$$

An error of the quadrature rule (1.3) is defined as

$$
R_{N}\left(\phi, p_{k}, t_{k}\right)=\sup _{0 \leq s \leq 2 \pi}\left|R_{N}\left(s, \phi, p_{k}, t_{k}\right)\right| .
$$

The error of the quadrature rule on class $\Psi$ is defined as

$$
R_{N}\left(\Psi, p_{k}, t_{k}\right)=\sup _{0 \leq s \leq 2 \pi} R_{N}\left(\phi, p_{k}, t_{k}\right)
$$

Define a functional $\zeta_{N}[\Psi]=\inf _{p_{k}, t_{k}} R_{N}\left(\Psi, p_{k}, t_{k}\right)$.

The quadrature rule with coefficients $p_{k}^{*}$ and nodes $t_{k}^{*}$ is optimal, asymptotically optimal, optimal with respect to order on class of functions $\Psi$ among all quadrature rules of the type (1.3) provided that $R_{N}\left(\Psi, p_{k}^{*}, t_{k}^{*}\right) / \zeta_{N}[\Psi]=1, \sim 1$, or $\asymp 1$.

1.2. Classes of functions. In this section, we will list several classes of functions which will be constantly used later. Some definitions we will take from [31].

A function $f$ defined on $A=[a, b]$ or $A=K$, where $K$ is a unit circle, satisfies a Hölder conditions with constant $M$ and exponent $\alpha$, or belongs to class $H_{\alpha}(M)$, $M \geq 0,0 \leq \alpha \leq 1$ if $\left|f\left(x^{\prime}\right)-f\left(x^{\prime \prime}\right)\right| \leq M\left|x^{\prime}-x^{\prime \prime}\right|^{\alpha}, x^{\prime}, x^{\prime \prime} \in A$.

More general is the class $H_{\alpha, \rho}(M)$. This consists of all functions $f(t)$ which can be represented as $f(t)=g(t) / \rho(t)$, where $g(t) \in H_{\alpha}(M), \rho(t)$ is a weight function.

Class $H_{\omega}(M)$, where $\omega(h)$ is a modulus of continuity, consists of all functions $f \in C(A)$ with the property $\left|f\left(x^{\prime}\right)-f\left(x^{\prime \prime}\right)\right| \leq M \omega\left(\left|x^{\prime}-x^{\prime \prime}\right|\right), x^{\prime}, x^{\prime \prime} \in A$.

Class $W^{r}(M)$ consists of functions $f(x) \in C(A)$ which have continuous derivatives $f^{\prime}, f^{\prime \prime}, \ldots, f^{(r-1)}$ on $A$, a piecewise continuous derivative $f^{(r)}$ on $A$ satisfying $\max _{x \in[a, b]}\left|f^{(r)}(x)\right| \leq M$.

Let $W_{\rho}^{r}(1)$ be the class of functions $f(t)$ which can be represented as $f(t)=$ $\varphi(t) / \rho(t)$, where $\varphi(t) \in W^{r}(1),\|\varphi\|_{C}=1, \rho(t)$ is a weight function.

The class of functions $W_{p}^{r}(M), r=1,2, \ldots, 1 \leq p \leq \infty$, consists of functions $f(x)$, defined on a segment $A=[a, b]$ or one $A=K$, that have continuous derivatives $f^{\prime}, f^{\prime \prime}, \ldots, f^{(r-1)}$, integrable derivative $f^{(r)}$ satisfying

$$
\left[\int_{A}\left|f^{(r)}(x)\right|^{p} d x\right]^{1 / p} \leq M
$$

Let $\Phi$ be the class of functions $f(x)$ that are defined on the segment $[0, a]$ and satisfy the conditions:

(1) $\lim _{x \rightarrow 0} f(x)=0$;

(2) $f(x)$ is almost increasing;

(3) $\sup _{x>0} 1 / f(x) \int_{0}^{x} f(s) / s d s=A_{f}<\infty$;

(4) $\sup _{x>0} x / f(x) \int_{0}^{x} f(s) / s d s=B_{f}<\infty$. 
A function $f\left(x_{1}, x_{2}, \ldots, x_{l}\right), l=2,3, \ldots$, defined on $A=\left[a_{1}, b_{1} ; a_{2}, b_{2} ; \ldots ; a_{l}, b_{l}\right]$ or $A=$ $K_{1} \times K_{2} \times \cdots \times K_{l}$, where $K_{i}, i=1,2, \ldots, l$, are unit circles satisfying Hölder conditions with constant $M$ and exponents $\alpha_{i}, i=1,2, \ldots, l$, or belongs to the class $H_{\alpha_{1}, \ldots, \alpha_{l}}(M)$, $M \geq 0,0 \leq \alpha_{i} \leq 1, i=1,2, \ldots, l$, if

$$
\left|f\left(x_{1}, \ldots, x_{l}\right)-f\left(y_{1}, \ldots, y_{l}\right)\right| \leq M\left(\left|x_{1}-y_{1}\right|^{\alpha_{1}}+\cdots+\left|x_{l}-y_{l}\right|^{\alpha_{l}}\right) .
$$

Let $\omega(h), \omega_{i}(h)$, where $i=1,2, \ldots, l, l=2,3, \ldots$, be a modulus of continuity.

The class $H_{\omega_{1}, \ldots, \omega_{l}}(M)$, consists of all functions $f \in C(A), A=\left[a_{1}, b_{1} ; a_{2}, b_{2} ; \ldots\right.$; $\left.a_{l}, b_{l}\right]$ or $A=K_{1} \times K_{2} \times \cdots \times K_{l}$, with a property

$$
\left|f\left(x_{1}, \ldots, x_{l}\right)-f\left(y_{1}, \ldots, y_{l}\right)\right| \leq M\left(\omega_{1}\left(\left|x_{1}-y_{1}\right|\right)+\cdots+\omega_{l}\left(\left|x_{l}-y_{l}\right|\right)\right) .
$$

Let $H_{j}^{\omega}(A), j=1,2,3, A=\left[a_{1}, b_{1} ; \ldots ; a_{l}, b_{l}\right]$, or $A=K_{1} \times K_{2} \times \cdots \times K_{l}, l=2,3, \ldots$, be the class of functions $f\left(x_{1}, \ldots, x_{l}\right)$ defined on $A$ and satisfying

$$
|f(x)-f(y)| \leq \omega\left(\rho_{j}(x, y)\right), \quad j=1,2,3,
$$

where $x=\left(x_{1}, \ldots, x_{l}\right), y=\left(y_{1}, \ldots, y_{l}\right), \rho_{1}(x, y)=\max _{1 \leq i \leq l}\left(\left|x_{i}-y_{i}\right|\right), \rho_{2}(x, y)=$ $\sum_{i=1}^{l}\left|x_{i}-y_{i}\right|, \rho_{3}(x, y)=\left[\sum_{i=1}^{l}\left|x_{i}-y_{i}\right|^{2}\right]^{1 / 2}$.

Let $Z_{j}^{\omega}(A), j=1,2,3$, be the class of functions $f\left(x_{1}, \ldots, x_{l}\right)$, defined on $A$ and satisfying $|f(x)+f(y)-2 f((x+y) / 2)| \leq \omega\left(\rho_{j}(x, y) / 2\right), j=1,2,3$.

Let $W^{r_{1}, \ldots, r_{l}}(M), l=2,3, \ldots$, be the class of functions $f\left(x_{1}, \ldots, x_{l}\right)$, defined on a domain $A$, which have continuous partial derivatives $\partial^{|v|} f\left(x_{1}, \ldots, x_{l}\right) / \partial x_{1}^{v_{1}} \cdots \partial x_{l}^{v_{l}}, 0<$ $|v| \leq r-1,|v|=v_{1}+\cdots+v_{l}, v_{i} \geq 0, i=1,2, \ldots, l, r=r_{1}+\cdots+r_{l}$, and all piece-continuous partial derivatives of order $r$ satisfying $\| \partial^{r} f\left(x_{1}, \ldots, x_{l}\right) /$ $\partial x_{1}^{r_{1}} \cdots \partial x_{l}^{r_{l}} \|_{C} \leq M$.

Let $A=\left[a_{1}, b_{1} ; a_{2}, b_{2} ; \ldots ; a_{l}, b_{l}\right]$ or $A=K_{1} \times K_{2} \times \cdots \times K_{l}, l=2,3, \ldots$. Let $C_{l}^{r}(M)$ be the class of functions $f\left(x_{1}, \ldots, x_{l}\right)$ which are defined in $A$ and which have continuous partial derivatives up to $r-1$ and a piecewise continuous partial derivatives of order $r$. The partial derivatives of order $r$ satisfy the conditions

$$
\left\|\frac{\partial^{r} f\left(x_{1}, \ldots, x_{l}\right)}{\partial x_{1}^{v_{1}} \cdots \partial x_{l}^{v_{l}}}\right\|_{C} \leq M
$$

for any $v=\left(v_{1}, \ldots, v_{l}\right)$, where $v_{i}, i=1,2, \ldots, l$ are integer and $\sum_{i=1}^{l} v_{i}=r$.

1.3. Preliminaries. In this paper, we will use an affirmation by S. Smolyak quoted from Bakhvalov's article [4].

LEMmA BY S. SMOLYAK. Set $L(f), L_{1}(f), \ldots, L_{N}(f)$ for linear functional and $\Omega$ for a convex centric symmetrical set with center of symmetry $\theta$ in the linear metric space. Then the numbers $D_{1}, \ldots, D_{N}$ exist and they are such that

$$
\sup _{f \in \Omega}\left|L(f)-\sum_{k=1}^{N} D_{k} L_{k}(f)\right|=R(T),
$$

that is, among the best methods there is the linear method.

In Smolyak lemma the following notations were used:

$$
T(f)=\left(L_{1}(f), \ldots, L_{N}(f)\right), \quad R(S, T)=\sup _{f \in \Omega}|L(f)-S(T(f))| .
$$


Here the functional $L(f)$ is calculated by the method $S$ in which the information $T(f)$ is used. An error of calculating $L(f)$ is given by $R(T)=\inf _{S} R(S, T)$.

Now we will describe some designations which will be used in this paper.

Let $f(t)$ be a function which is defined on the segment $[a, b]$ and belongs to the class of functions $W^{r}(M)$. Let $c \in[a, b]$. An expression $T_{r-1}(f,[a, b], c)$ is a designation of a segment of Taylor series

$$
T_{r-1}(f,[a, b], c)=f(c)+\frac{1}{1 !} f^{(1)}(c)(t-c)+\cdots+\frac{1}{(r-1) !} f^{(r-1)}(c)(t-c)^{r-1} .
$$

Let $f\left(x_{1}, \ldots, x_{l}\right) \in W^{r, \ldots, r}(M), r=1,2, \ldots, x \in D=\left[a_{1}, b_{1} ; \ldots ; a_{l}, b_{l}\right]$. Let $c \in D$. Let $T_{r}(f, D, c)$ be a segment of the Taylor series

$$
T_{r}(f, D, c)=f(c)+\frac{1}{1 !} d f(c)+\cdots+\frac{1}{r !} d^{r} f(c) .
$$

Let $f\left(x_{1}, x_{2}\right) \in W^{r, s}(M), x=\left(x_{1}, x_{2}\right) \in D=[a, b ; c, d]$. Let $\bar{a} \in[a, b], \bar{c} \in[c, d]$. Let $T_{r s}(f, D,(\bar{a}, \bar{b}))$ be a segment of Taylor series

$$
T_{r s}(f, D,(\bar{a}, \bar{b}))=T_{r}\left(T_{s}\left(f\left(x_{1}, x_{2}\right),[c, d], \bar{c}\right),[a, b], \bar{a}\right) .
$$

Let $D_{r}(t)$ be a function

$$
D_{r}(t)=\frac{1}{2^{r} \pi^{r}} \sum_{k=1}^{\infty} \frac{1}{k^{r}} \cos \left(2 \pi k t-\frac{\pi r}{2}\right) .
$$

Favar constant $K_{r}$ is defined as

$$
K_{r}=\frac{4}{\pi} \sum_{k=1}^{\infty}(-1)^{k(r+1)} \frac{1}{(2 k+1)^{r+1}}, \quad r=0,1, \ldots
$$

Let $R_{r q}(x)=x^{r}+\sum_{k=0}^{r-1} a_{k} x^{k}$ be a polynomial of degree $r$ of the least derivation from zero in the space $L_{q}[-1,1]$.

Let $R_{r q}(a ; h ; x)$ be a polynomial $x^{r}+\sum_{k=0}^{r-1} a_{k} x^{k}$ such that

$$
\int_{a-h}^{a+h}\left|R_{r q}(a ; h ; x)\right|^{q} d x=\min _{a_{0}, \ldots, a_{r-1}} \int_{a-h}^{a+h}\left|x^{r}+\sum_{k=0}^{r-1} a_{k} x^{k}\right|^{q} d x .
$$

Let $f(t)$ be a function which is defined on the segment $[a, b]$ and belongs to the class of functions $W_{p}^{r}(M)$. Now we construct the special polynomial for approximation of the function $f(t)$ on the segment $[a, b]$. This polynomial will be used for constructing optimal quadrature rules for singular and Hadamard integrals.

We introduce a polynomial $\tilde{f}(\tau,[a, b])$ corresponding to the formula

$$
\begin{aligned}
\tilde{f}(\tau,[a, b]) & =\sum_{k=0}^{r-1}\left(\frac{f^{(k)}(a)}{k !}(\tau-a)^{k}+B_{k} \delta^{(k)}(b)\right), \\
\delta(\tau) & =f(\tau)-\sum_{k=0}^{r-1} \frac{f^{(k)}(a)}{k !}(\tau-a)^{k} .
\end{aligned}
$$


Coefficients $B_{k}$ are determined from the equality

$$
(b-t)^{r}-\sum_{j=0}^{r-1} \frac{B_{j} r !(b-a)}{(r-j-1) !}(b-a)^{r-j-1}=(-1)^{r} R_{r q}(c, h, t),
$$

where $R_{r q}(c, h, t)=t^{r}+\sum_{k=0}^{r-1} a_{k} t^{k}$ is the polynomial of degree $r$ of least deviation from zero in the space $L_{q}[a, b](1 / p+1 / q=1), c=(a+b) / 2, h=(b-a) / 2$.

Let $f \in W_{p}^{r}(M,[a, b]), r=1,2, \ldots, 1 \leq p \leq \infty$. Divide the segment $[a, b]$ into smaller segments $\Delta_{k}=\left[t_{k}, t_{k+1}\right], k=0,1, \ldots, n-1 ; t_{k}=a+(b-a) k / n, k=0,1, \ldots, n$. Approximate the function $f(t)$ on the segment $\Delta_{k}$ by the polynomial $\tilde{f}\left(t, \Delta_{k}\right), k=0,1, \ldots, n-1$, which was described above. A local spline is defined on the segment $[a, b]$ and consists of the polynomials $\tilde{f}\left(t, \Delta_{k}\right), k=0,1, \ldots, n-1$, and is denoted by $\tilde{f}(t)$.

Let $f(t)$ be a function defined on the segment $[a, b]$ and belongs to class of functions $W_{p}^{r}(M,[a, b]), r=1,2, \ldots, 1 \leq p \leq \infty$. Let $D_{n, r, p}\left(f^{(l)}\left(t_{j}\right)\right), 0 \leq l \leq r-1$ be a difference operator with approximate value $f^{(l)}\left(t_{j}\right)$ to within $A n^{-2(r-l)}$. This operator is constructed by values $f\left(v_{k}\right), k=1,2, \ldots, r+1$, and one is exact for the polynomials of order $r-1$.

Let $f\left(t_{1}, t_{2}\right) \in W^{r, s}(M, D), r, s=1,2, \ldots, D=\left[a_{1}, b_{1} ; a_{2}, b_{2}\right]$. Let $D_{m, n}^{r, s}\left(f^{(k, l)}\left(\tau_{1}, \tau_{2}\right)\right)$, $1 \leq k \leq r-1,1 \leq l \leq s-1$, be a difference operator with approximate value $f^{(k, l)}\left(\tau_{1}, \tau_{2}\right)$ to within $A m^{-2(r-l)} n^{-2(s-l)}$. The operator $D_{m, n}^{r, s}$ must be exact for the polynomials of $t_{1}^{v} t_{2}^{w}, v=0,1, \ldots, r-1, w=0,1, \ldots, s-1$ and one must use values $f\left(\zeta_{i}, \xi_{j}\right)$, $i=1,2 \ldots, r+1, j=1,2, \ldots, s+1$.

We describe one way of constructing an operator $D_{n, r, p}$.

Assume we should like to construct the operator $D_{n, r, p}$ for approximation of the value $f^{(l)}(0), 0 \leq l \leq r-1$. Let $h=n^{-2}$ be a small number. We approximate the function $f(t)$ on the segment $[0, h]$ with the Lagrange interpolation polynomials on $r+1$ nodes $v_{k} \in[0, h], k=1,2, \ldots, r+1$. This interpolation polynomial is one kind of the operator $D_{n, r, p}$. Using theory of approximation $[34,35]$ we can conclude that operator $D_{n, r, p}$ has all needed properties.

An operator $D_{m, n}^{r, s}$ can be constructed by similar ways.

Let $f(t) \in W_{p}^{r}(M,[a, b]), r=1,2, \ldots, 1 \leq p \leq \infty$. Let

$$
Q_{n, r, p}(f,[a, b])=\sum_{k=1}^{n} p_{k} f\left(t_{k}\right)
$$

be the asymptotically optimal quadrature rule for calculation of the integral $\int_{a}^{b} f(t) d t$.

Let $f\left(t_{1}, t_{2}\right) \in W^{r, s}(M, D), r, s=1,2, \ldots, D=\left[a_{1}, b_{1} ; a_{2}, b_{2}\right]$. Let

$$
Q_{n_{1}, n_{2}}^{r, s}\left(f ;\left[a_{1}, b_{1} ; a_{2}, b_{2}\right]\right)=\sum_{k_{1}=1}^{n_{1}} \sum_{k_{2}=1}^{n_{2}} p_{k_{1} k_{2}} f\left(t_{k_{1} k_{2}}\right)
$$

be the asymptotically optimal quadrature rule for calculation of the integral

$$
\int_{a_{1}}^{b_{1}} \int_{a_{2}}^{b_{2}} f\left(t_{1}, t_{2}\right) d t_{1} d t_{2} .
$$


We describe one of methods of construction of a functional $Q_{n, r, p}(f ;[a, b])$. It is well known [36], that Euler-Maclaurin quadrature rule

$$
\int_{a}^{b} f(x) d x=a_{0} f(a)+\sum_{k=0}^{m} p_{k} f\left(x_{k}\right)+b_{0} f(b)+\sum_{v=1}^{r-1} a_{v}\left(f^{(v)}(b)-f^{(v)}(b)\right)+R_{m}(f)
$$

is optimal on class $W_{p}^{r}(1)$. Approximating derivatives $f^{(v)}(b)$ and $f^{(v)}(a)$ by the difference operators $D_{n, r, p}\left(f^{(v)}(b)\right)$ and $D_{n, r, p}\left(f^{(v)}(a)\right)$ we receive the asymptotically optimal quadrature rule

$$
\begin{aligned}
Q_{n, r, p}(f ;[a, b])= & a_{0} f(a)+\sum_{k=1}^{m} p_{k} f\left(x_{k}\right)+b_{0} f(b) \\
& +\sum_{v=1}^{r-1} a_{v}\left(D_{n, r, p}\left(f^{(v)}(b)\right)-D_{n, r, p}\left(f^{(v)}(a)\right)\right) .
\end{aligned}
$$

The asymptotically optimal quadrature rules $Q_{n_{1}, n_{2}}^{r, s}\left(f,\left[a_{1}, b_{1} ; a_{2}, b_{2}\right]\right)$ are constructed by similar ways.

A polynomial $P_{r}(f,[a, b])$ that interpolated the function $f(t)$ on the segment $[a, b]$ is constructed as follows. Denote by $\zeta_{k}, k=1,2, \ldots, r$, the roots of the Legendre polynomial of degree $r$. We map a segment $\left[\zeta_{1}, \zeta_{r}\right] \in[-1,1]$ onto $[a, b]$ so that the points $\zeta_{1}$ and $\zeta_{r}$ map to $a$ and $b$, respectively. Images of the points $\zeta_{i}$ under this mapping are denoted by $\zeta_{i}^{\prime}, i=1,2, \ldots, r$. Using the points of $\zeta_{i}^{\prime}, i=1,2, \ldots, r$, we construct the interpolation polynomial $P_{r}(f,[a, b])$ of degree $r-1$.

The abbreviation q.r. means quadrature rule. The symbol [a] means the greatest integer in $a$.

1.4. Short reviews on approximate methods for calculating singular and hypersingular integrals. Singular and hypersingular integrals of the forms

$$
\begin{aligned}
I f & =\int_{-1}^{1} \frac{f(t)}{t} d t \\
H f & =\frac{1}{2 \pi} \int_{0}^{2 \pi} f(\sigma) \operatorname{ctg} \frac{\sigma-s}{2} d \sigma \\
K f & =\int_{-1}^{1} \frac{\omega(\tau) f(\tau)}{\tau-t} d \tau \\
J f & =\int_{0}^{2 \pi} \int_{0}^{2 \pi} f\left(\sigma_{1}, \sigma_{2}\right) \operatorname{ctg} \frac{\sigma_{1}-s_{1}}{2} \operatorname{ctg} \frac{\sigma_{2}-s_{2}}{2} d \sigma_{1} d \sigma_{2}, \\
L f & =\int_{-1}^{1} \int_{-1}^{1} \frac{\omega_{1}\left(\tau_{1}\right) \omega_{2}\left(\tau_{2}\right) f\left(\tau_{1}, \tau_{2}\right)}{\left(\tau_{1}-t_{1}\right)\left(\tau_{2}-t_{2}\right)} d \tau_{1} d \tau_{2}, \\
M f & =\int_{D} \frac{p(\theta) f(u)}{r(u, v)} d u, \\
A f & =\int_{-1}^{1} \frac{f(t)}{t^{v}} d t, \quad B f=\int_{-1}^{1} \frac{f(t)}{|t|^{v+\lambda}} d t, \quad v=1,2,3, \ldots, 0<\lambda<1, \\
C f & =\int_{-1}^{1} \frac{f(t) d t}{(t-s)^{v}}, \quad v=2,3, \ldots,
\end{aligned}
$$




$$
\begin{aligned}
& D f=\int_{-1}^{1} \int_{-1}^{1} \frac{f\left(t_{1}, t_{2}\right) d t_{1} d t_{2}}{\left(t_{1}-s_{1}\right)^{v_{1}}\left(t_{2}-s_{2}\right)^{v_{2}}}, \quad v_{1}, v_{2}=2,3, \ldots, \\
& E f=\int_{-1}^{1} \int_{-1}^{1} \frac{f\left(t_{1}, t_{2}\right) d t_{1} d t_{2}}{\left(\left(t_{1}-s_{1}\right)^{2}+\left(t_{2}-s_{2}\right)^{2}\right)^{v}}, \quad v=2,3, \ldots, v,
\end{aligned}
$$

where $\theta=(u-v) / r(u, v), u=\left(u_{1}, u_{2}\right), v=\left(v_{1}, v_{2}\right), r(u, v)=\left[\left(u_{1}-v_{1}\right)^{2}+\left(u_{2}-\right.\right.$ $\left.\left.v_{2}\right)^{2}\right]^{1 / 2}, D=[-1,1 ;-1,1]$, play important role in fields like aerodynamics, electrodynamics, the theory of elasticity and other areas of physics and engineering sciences.

One of the first publications devoted to approximate evaluation of singular integrals with fixed singularity of type (1.26) was [29] in which the classical Gauss quadrature rule was applied to the integral

$$
I f=\int_{0}^{1} \frac{f(\tau)-f(0)}{\tau} d \tau .
$$

Optimal, asymptotically optimal, and optimal with respect to order quadrature rules for calculating singular integrals of type (1.26) was investigated in the series of the papers by Boikov. These results and references can be found in [5, 6, 8, 9].

Asymptotically optimal and optimal with respect to order quadrature rules for calculating singular integrals of type (1.26) were diffused in [11] to the hypersingular integrals as (1.32).

A great number of publications is devoted to numerical methods of the calculation of singular integrals as (1.27) and (1.28).

For numerical evaluation of singular integrals as (1.27) there are often constructed the following quadrature rules. They approximate the integrand function $f(t)$ by the interpolated polynomial $P_{2 n}[f]$ with nodes $s_{k}=2 k \pi /(2 n+1), k=0,1, \ldots, 2 n+1$, (or other nodes) and introduce a quadrature rule

$$
H f=\frac{1}{2 \pi} \int_{0}^{2 \pi} f(\sigma) \frac{\sigma-s}{2} d \sigma=\frac{1}{2 \pi} \int_{0}^{2 \pi} P_{2 n}[f](\sigma) \frac{\sigma-s}{2} d \sigma+R_{n} .
$$

The integral in the right-hand side is calculated exactly.

Similar quadrature rules are constructed for the singular integrals as (1.28)

$$
K f=\int_{-1}^{1} \frac{\omega(\tau) f(\tau) d \tau}{\tau-t}=\int_{-1}^{1} \frac{\omega(\tau) P_{n-1}[f](\tau) d \tau}{\tau-t}+R_{n} .
$$

The $P_{n-1}[f](t)$ is an interpolated polynomial with nodes $-1 \leq t_{1}<t_{2}<\cdots<t_{n} \leq 1$. These procedures have been investigated in [15, 16, 22, 23, 27, 33, 40].

Instead of the interpolation polynomials for the approximation of the integrand function there often are used partial sums of Fourier series, Vallee-Poussin, BernsteinRogozinski, Fejer, Abel-Poisson, Cesaro sums. Some results in this direction are given in [45].

The discrete vortex method detailed for the solution of many tasks of aerodynamics was used for the numerical calculation of singular integrals as (1.27), (1.28), (1.29), and (1.30). Explicit presentation of discrete vortex method is given in [30]. 
For evaluation of the singular integrals as (1.27) and (1.28) many authors approximate an integrand function with different splines. Investigation in this direction can be found in $[8,9,39]$.

Evaluation of singular integrals with Cauchy kernel based on approximating the integrand function by Whittaker cardinal or Sinc functions was investigated in [44].

Quadrature rules with the highest trigonometrical precision for singular integrals with Hilbert kernel and weight $\omega$ was discussed in $[18,19]$.

For the evaluation of singular integrals many authors use the method of subtraction of singularity. They write

$$
K[f, t]=\int_{-1}^{1} \frac{\omega(\tau)(f(\tau)-f(t))}{\tau-t} d \tau+f(t) \int_{-1}^{1} \frac{\omega(\tau)}{\tau-t} d \tau
$$

and approximate the integral on the right-hand side using classical quadrature rules. Investigation in this direction can be found in [17, 27].

In the theory of numerical approximation of Cauchy type integrals, three kinds of Gaussian quadrature rules have been investigated.

Let a function $f(t)$ be interpolated by the polynomial $P_{n-1}[f]$ of degree $n-1$ using the zeroes of the $n$th Jacobi polynomial with the weight function $\omega(t)$ as interpolation nodes. Then $K\left[P_{n-1} f, t\right]$ is the Gaussian quadrature rule for the Cauchy principal value integral.

The results on the Gaussian quadrature rules can be found in [17, 20, 21, 22, 25].

On the other hand, the integral $K[f, t]$ can be represented as (1.39). Then the first integral on the right-hand side of (1.39) is a Riemann integral. It can be approximated with Gaussian quadrature rules for Riemann integrals. The resulting approximation for $K[f, t]$ is called the modified Gaussian quadrature rules for the Cauchy principal value integral. Results on the modified Gaussian quadrature rules can be found in [20, 22, 24].

The Gaussian quadrature rule of the third kind

$$
K[f(t)]=\int_{-1}^{1} \frac{\omega(\tau)\left(P_{n-1}[f](\tau)-P_{n-1}[f](t)\right)}{\tau-t} d \tau+f(t) \int_{-1}^{1} \frac{\omega(\tau)}{\tau-t} d \tau
$$

was proposed in [18].

For the evaluation of polysingular integrals as (1.30) and (1.31) many authors replaced a function $f$ on the interpolated polynomials or splines. These methods were considered in $[6,8,46]$.

The uniform convergence with respect to the parameters $t_{1}$ and $t_{2}$ of the numerical methods for evaluating the Cauchy principal value integral (1.30), where $\omega_{1}, \omega_{2}$ are the Jacobi weight functions $\omega_{i}(t)=(1-t)^{\alpha_{i}}(1+t)^{\beta_{i}}, \alpha_{i}, \beta_{i}>-1, i=1,2$, was studied in [41].

The numerical methods of the evaluation of singular and polysingular integrals on Hardy spaces are given in $[8,10]$.

From this short review it follows that many methods for calculating singular integrals exist. It is necessary to find a criteria for the comparison of these methods. One of these criterions is the optimality of algorithms. 
Optimal with respect to order quadrature rule for the evaluation integral as (1.27) on Hölder and Sobolev classes of functions was constructed in [26]. Later asymptotically optimal and optimal with respect to order quadrature rule for the evaluation integrals as (1.27), (1.28), (1.29), (1.30), and (1.31) on Hölder and Sobolev classes of functions was constructed by Boikov. These results were summed in [5, 6, 8, 9] which consist of bibliography on numerical methods of the evaluation of singular and hypersingular integrals.

Asymptotically optimal and optimal with respect to order quadrature rules for the calculation of singular integrals was diffused in [11] to hypersingular integrals as (1.32), (1.33), (1.34), and (1.35).

2. Singular integrals with fixed singularity. In this section, we give optimal, asymptotically optimal, optimal with respect to order quadrature rules for calculating onedimensional singular integrals with fixed singularity.

2.1. Optimal algorithms for calculating singular integrals with fixed singularity. Up to now we know only four statements of optimal algorithms of calculating singular integrals with fixed singularity.

We consider a singular integral

$$
I f=\int_{-1}^{1} \frac{f(\tau)}{\tau} d \tau
$$

We will compute the integral $I f$ by a quadrature rule as

$$
I f=\sum_{k=-N}^{N}{ }^{\prime} p_{k} f\left(t_{k}\right)+R_{N}\left(f, p_{k}, t_{k}\right),
$$

where $-1 \leq t_{-N}<\cdots<t_{-1} \leq 0 \leq t_{1}<\cdots<t_{N} \leq 1$, prime in summation indicate that $k \neq 0$.

We will consider the quadrature rules as (2.2) under two assumptions:

(1) $t_{ \pm N}= \pm 1$, such that formula (2.2) is a Markov quadrature rule;

(2) $t_{N} \geq-1, t_{N} \leq 1$.

TheOrem 2.1 (see $[6,8]$ ). Let $\Psi=W^{1}(1)$. Among all possible Markov quadrature rules of type (2.2) the quadrature rule

$$
\begin{aligned}
I f= & \sum_{k=1}^{N-1} 2 \ln \frac{k+1}{k}\left(f\left(\frac{k(k+1)}{N(N+1)}\right)-f\left(-\frac{k(k+1)}{N(N+1)}\right)\right) \\
& +(f(1)-f(-1)) \ln \frac{N+1}{N}+R_{N}
\end{aligned}
$$

is optimal. The error of the quadrature rule (2.3) is equal to $R_{n}(\Psi)=2 \ln (1+1 / N)$.

THEOREM 2.2 (see $[6,8]$ ). Let $\Psi=W^{1}(1)$. Among all possible quadrature rules of type (2.2) the quadrature rule

$$
I f=\sum_{k=1}^{N} 2 \ln \frac{k+1}{k}\left(f\left(\frac{k(k+1)}{(N+1)^{2}}\right)-f\left(-\frac{k(k+1)}{(N+1)^{2}}\right)\right)+R_{N}
$$

is optimal. The error of the quadrature rule (2.4) is equal to $R_{N}(\Psi)=2 /(N+1)$. 
THEOREM 2.3 (see [37]). Let $\Psi=H_{1}(1)$. Among all possible Markov quadrature rules of type (2.2) the quadrature rule (2.3) is optimal.

THEOREM 2.4 (see [37]). Let $\Psi=H_{1}(1)$. Among all possible quadrature rules of type (2.2) the quadrature rule (2.4) is optimal.

Proofs of TheOrems. To make some notices relating to the proofs of the theorems.

First of all we assume that the quadrature rule (2.2) is strictly for polynomials of order $r-1$ in case applying it to functions of the $W^{r}(1)$ class.

We expand the function $\phi(t)$ by the Taylor formula with remainder term in the integral form

$$
\begin{array}{ll}
\phi(t)=\sum_{k=0}^{r-1} \frac{\phi^{(k)}(0)}{k !} t^{k}+\frac{1}{(r-1) !} \int_{0}^{1} K_{r}(t-s) \phi^{(r)}(s) d s \quad \text { for } t \geq 0, \\
\phi(t)=\sum_{k=0}^{r-1} \frac{\phi^{(k)}(0)}{k !} t^{k}+\frac{1}{(r-1) !} \int_{0}^{-1} \bar{K}_{r}(t-s) \phi^{(r)}(s) d s & \text { for } t \leq 0,
\end{array}
$$

where

$$
\begin{aligned}
& K_{r}(u)= \begin{cases}u^{r-1} & \text { for } u \geq 0, \\
0 & \text { for } u<0,\end{cases} \\
& \bar{K}_{r}(u)= \begin{cases}u^{r-1} & \text { for } u \leq 0, \\
0 & \text { for } u>0 .\end{cases}
\end{aligned}
$$

Since the quadrature rule (2.2) is exact for polynomials of degree not higher than $r-1$ hence

$$
\begin{aligned}
\int_{-1}^{1} \frac{\phi(\tau)}{\tau} d \tau-\sum_{k=-N, k \neq 0}^{N} p_{k} \phi\left(t_{k}\right) \\
=\frac{1}{(r-1) !} \int_{-1}^{1} \frac{1}{\tau}\left[\int_{0}^{\tau}(\tau-t)^{r-1} \phi^{(r)}(t) d t\right] d \tau \\
\quad-\sum_{k=-N, k \neq 0}^{N} \frac{p_{k}}{(r-1) !} \int_{0}^{t_{k}}\left(t_{k}-t\right)^{r-1} \phi^{(r)}(t) d t \\
=\frac{1}{(r-1) !} \int_{0}^{1} \phi^{(r)}(t)\left[\int_{0}^{1} \frac{K_{r}(\tau-t)}{\tau} d \tau-\sum_{k=1}^{N} p_{k} K_{r}\left(t_{k}-t\right)\right] d t \\
\quad+\frac{1}{(r-1) !} \int_{-1}^{0} \phi^{(r)}(t)\left[\int_{0}^{-1} \frac{\bar{K}_{r}(\tau-t)}{\tau} d \tau-\sum_{k=-N}^{-1} p_{k} \bar{K}_{r}\left(t_{k}-t\right)\right] d t
\end{aligned}
$$

Thus the error of the quadrature rule (2.2) on the function class $W^{r}(1)$ is defined by the inequality

$$
\left|R_{N}\right| \leq \frac{2}{(r-1) !}\left|\int_{0}^{1} \phi^{(r)}(t)\left[\int_{0}^{1} \frac{K_{r}(\tau-t)}{\tau} d \tau-\sum_{k=1}^{N} p_{k} K_{r}\left(t_{k}-t\right)\right] d t\right|
$$


Proof of TheORem 2.1. It follows from the theorem conditions that $r=1, t_{-N}=$ $-1, t_{N}=1$. In this case

$$
\begin{aligned}
\left|R_{N}\right| & \leq 2\left|\int_{0}^{1} \phi^{\prime}(t)\left[\int_{0}^{1} \frac{K_{1}(\tau-t)}{\tau} d \tau-\sum_{k=1}^{N} p_{k} K_{1}\left(t_{k}-t\right)\right] d t\right| \\
& \leq 2\left|\int_{0}^{1}\right| \int_{0}^{1} \frac{K_{1}(\tau-t)}{\tau} d \tau-\sum_{k=1}^{N} p_{k} K_{1}\left(t_{k}-t\right)|d t| \\
& =2 \int_{0}^{1}\left|-\ln t-\sum_{k=1}^{N} p_{k} K_{1}\left(t_{k}-t\right)\right| d t .
\end{aligned}
$$

We find the nodes $t_{k}$ and the weights $p_{k}$ from the integral minimality conditions assuming $t_{0}=0$

$$
\begin{aligned}
A_{n} & =\int_{0}^{1}\left|-\ln t-\sum_{k=1}^{N} p_{k} K_{1}\left(t_{k}-t\right)\right| d t \\
& =\int_{0}^{t_{1}}\left|-\ln t-M_{1}\right| d t+\int_{t_{1}}^{t_{2}}\left|-\ln t-M_{2}\right| d t+\cdots+\int_{t_{N-1}}^{1}\left|-\ln t-M_{N}\right| d t \\
& =\int_{0}^{t_{1}^{\prime}}\left(-\ln t-M_{1}\right) d t+\int_{t_{1}^{\prime}}^{t_{1}}\left(M_{1}+\ln t\right) d t+\cdots+\int_{t_{N-1}}^{t_{N}^{\prime}}\left(-\ln t-M_{N}\right) d t+\int_{t_{N}^{\prime}}^{1}\left(M_{N}+\ln t\right) d t,
\end{aligned}
$$

where $t_{k}^{\prime} \in\left(t_{k}, t_{k+1}\right)$.

We differentiate the expression $A_{N}$ with respect to $t_{i}, t_{i}^{\prime}, M_{i}$ and assume the obtained expressions are equal to zero. As a result we have the equations system

$$
\begin{aligned}
& \frac{\partial A_{N}}{\partial t_{i}}=M_{i}+2 \ln t_{i}+M_{i+1}=0, \quad i=1,2, \ldots, N-1 ; \\
& \frac{\partial A_{N}}{\partial t_{i}^{\prime}}=-2 M_{i}-2 \ln t_{i}^{\prime}=0, \quad i=1,2, \ldots, N-1, N ; \\
& \frac{\partial A_{N}}{\partial M_{i}}=-2 t_{i}^{\prime}+t_{i}+t_{i-1}=0, \quad i=1,2, \ldots, N-1, N .
\end{aligned}
$$

We transform the equations of system (2.11) to the following form:

$$
\begin{aligned}
\ln t_{i} & =-\frac{\left(M_{i}+M_{i+1}\right)}{2}, \quad i=1,2, \ldots, N-1 ; \\
M_{i} & =-\ln t_{i}^{\prime}, \quad i=1,2, \ldots, N-1, N \\
t_{i}^{\prime} & =\frac{\left(t_{i}+t_{i-1}\right)}{2}, \quad i=1,2, \ldots, N-1, N .
\end{aligned}
$$

Hence

$$
\begin{gathered}
\ln t_{i}=\frac{\left(\ln t_{i}^{\prime}+\ln t_{i+1}^{\prime}\right)}{2} \quad i=1,2, \ldots, N-1 \\
t_{i}^{\prime}=\frac{\left(t_{i}+t_{i-1}\right)}{2} \quad i=1,2, \ldots, N-1, N
\end{gathered}
$$


It follows that

$$
\begin{aligned}
t_{i}^{2} & =t_{i}^{\prime} t_{i+1}^{\prime}, \quad i=1,2, \ldots, N-1 ; \\
t_{i}^{2} & =\frac{t_{i}+t_{i-1}}{2} \frac{t_{i+1}+t_{i}}{2}, \quad i=1,2, \ldots, N-1 ; \\
4 t_{i}^{2} & =\left(t_{i}+t_{i-1}\right)\left(t_{i+1}+t_{i}\right) .
\end{aligned}
$$

We express $t_{i}(i=2, \ldots, N)$ by means of $t_{1}$ taking into account $t_{0}=0$. It follows from formula (2.14) that correctness of the recurrence relations is

$$
t_{i+1}=\frac{\left(3 t_{i}^{2}-t_{i} t_{i-1}\right)}{\left(t_{i}+t_{i-1}\right)}, \quad i=1,2, \ldots, N-1 .
$$

Using formula (2.15) we obtain

$$
t_{2}=3 t_{1}=(1+2) t_{1}, \quad t_{3}=6 t_{1}=(1+2+3) t_{1}, \quad t_{4}=10 t_{1}=(1+2+3+4) t_{1} .
$$

The mathematical induction method makes it possible to prove that $t_{n}=(1+n) \times$ $n t_{1} / 2$. In fact this formula is valid for $n=2,3,4$.

Let it holds for $n$. We show that it will be valid for $n+1$. Then

$$
t_{n+1}=\frac{\left(3 t_{n}^{2}-t_{n} t_{n-1}\right)}{\left(t_{n}+t_{n-1}\right)}=\frac{(n+2)(n+1) t_{1}}{2}
$$

and the formula is proved. Now from the request $t_{N}=1$ we find that $t_{1}=2 / N(N+$ 1 ). Having known the values $t_{i}^{\prime}=i^{2} t_{1} / 2$ it is easy to obtain $M_{i}=-\ln \left(i^{2} t_{1} / 2\right)=$ $-\ln \left(i^{2} / N(N+1)\right), i=1,2, \ldots, N$. The coefficients $p_{i}$ of the optimal quadrature rule can be determined with respect to the constants $M_{i}$. Really,

$$
p_{N}=M_{N}, p_{N-1}=M_{N-1}-M_{N}, p_{N-2}=M_{N-2}-M_{N-1}, \ldots, p_{1}=M_{1}-M_{2} .
$$

From here

$$
p_{N}=-\ln \left(\frac{N}{(N+1)}\right), \quad p_{k}=-2 \ln \left(\frac{k}{(k+1)}\right), \quad k=1,2, \ldots, N-1 .
$$

So we received the quadrature rule (2.3).

It is not difficult to estimate the value of its error

$$
\begin{aligned}
\left|R_{N}\right| \leq & 2 \sum_{k=1}^{N-1}\left|\int_{t_{k}}^{t_{k+1}}\left(\phi(\tau)-\phi\left(t_{k+1}^{\prime}\right)\right) \tau^{-1} d \tau\right|+\left|\int_{t_{-1}}^{t_{1}} \phi(\tau) \tau^{-1} d \tau\right| \\
\leq & 2\left[\frac{1}{N(N+1)}+\sum_{k=1}^{N-1}\left[t_{k} \ln \frac{t_{k}^{2}}{t_{k}^{\prime} t_{k+1}^{\prime}}+\left(t_{k}^{\prime}+t^{\prime}{ }_{k+1}-2 t_{k}\right)\right]\right. \\
& \left.\quad-\ln \frac{N^{2}}{N(N+1)}-\left(1-\frac{N^{2}}{N(N+1)}\right)\right] \\
= & 2 \ln \left(1+\frac{1}{N}\right) .
\end{aligned}
$$


In order to prove the optimality of constructing the quadrature rule it is necessary to point out the function $\phi(t)$ for which

$$
\left|R_{N}(\phi)\right|=2 \int_{0}^{1}\left|\ln t-\sum_{k=1}^{N} p_{k} K_{1}\left(t_{k}-t\right)\right| d t .
$$

A function $\phi(t)$ determined by the formula $\phi(t)=\min _{k}\left|t-t_{k}\right|, k=0,1, \ldots, N-1, N$, can be taken in the capacity of such function. This completes the proof.

Proof OF TheOrem 2.2. In principle this proof is similar to that of Theorem 2.1. As in the proof of Theorem 2.1, the quadrature rule is defined by the inequality (2.9). Since in this case $t_{N}$ must not be equal to 1 then $A_{N}$ must be presented in the form

$$
\begin{aligned}
A_{N}= & \int_{0}^{t_{1}}\left|-\ln t-M_{1}\right| d t+\int_{t_{1}}^{t_{2}}\left|-\ln t-M_{2}\right| d t+\cdots \\
& +\int_{t_{N-1}}^{t_{N}}\left|-\ln t-M_{N}\right| d t+\int_{t_{N}}^{1}|\ln t| d t \\
= & \int_{0}^{t_{1}^{\prime}}\left(-\ln t-M_{1}\right) d t+\int_{t_{1}^{\prime}}^{t_{1}}\left(M_{1}+\ln t\right) d t \\
& +\cdots+\int_{t_{N-1}}^{t_{N}^{\prime}}\left(-\ln t-M_{N}\right) d t+\int_{t_{N}^{\prime}}^{t_{N}}\left(M_{N}+\ln t\right) d t+\int_{t_{N}}^{1}-\ln t d t .
\end{aligned}
$$

Having minimized $A_{N}$ with respect to $t_{k}, t_{k}^{\prime}$ and $M_{k}$ we arrive at the system of equations

$$
\begin{aligned}
& \frac{\partial A_{N}}{\partial t_{i}}=M_{i}+2 \ln t_{i}+M_{i+1}=0, \quad i=1,2, \ldots, N-1 ; \\
& \frac{\partial A_{N}}{\partial t_{N}}=M_{N}+2 \ln t_{N}=0 ; \\
& \frac{\partial A_{N}}{\partial t_{i}^{\prime}}=-2 M_{i}-2 \ln t_{i}^{\prime}=0, \quad i=1,2, \ldots, N-1, N ; \\
& \frac{\partial A_{N}}{\partial M_{i}}=-2 t_{i}^{\prime}+t_{i}+t_{i-1}=0, \quad i=1,2, \ldots, N,
\end{aligned}
$$

that differs from the system of (2.11) only by adding the equation

$$
\frac{\partial A_{N}}{\partial t_{N}}=M_{N}+2 \ln t_{N}=0
$$

The solution of this system is not different from the solution of the equations system (2.11) therefore is missing the intermediate evaluations. So we reduce the final result: $t_{k}=k(k+1) /(N+1)^{2}, t_{k}^{\prime}=k^{2} /(N+1)^{2}, M_{k}=-2 \ln (k /(N+1)), k=1,2, \ldots, N$.

Hence the optimal quadrature rule has the meaning (2.4). So it is easy to see that the error of this quadrature rule is equal to the value $2 /(N+1)$. This completes the proof. 


\subsection{Asymptotically optimal algorithms on the class $H_{\alpha}$}

2.2.1. Integrals on finite segments. Consider the singular integrals (2.1) on Hölder class of functions. As a method of evaluation we use a quadrature rule q.r.

$$
I \varphi=\sum_{k=-N}^{N}{ }^{\prime} p_{k} \varphi\left(t_{k}\right)+R_{N}
$$

where $-1 \leq t_{-N}<\cdots<t_{-1} \leq 0 \leq t_{1}<\cdots<t_{N} \leq 1$, is prime in summation indicates that $k \neq 0$.

Input a quadrature rule

$$
I \varphi=\sum_{k=-N}^{N-1} " \varphi\left(t_{k}^{\prime}\right) \ln \left(\frac{t_{k+1}}{t_{k}}\right)+R_{N},
$$

where $t_{ \pm k}= \pm(k / N)^{(1+\alpha) / \alpha}, t_{k}^{\prime}=\left(t_{k}+t_{k+1}\right) / 2, k=1,2, \ldots, N-1, t_{-k}^{\prime}=\left(t_{-k}+t_{-k+1}\right) / 2$, $k=2,3, \ldots, N$, is double prime in summation indicates that $k \neq 0,-1$.

TheOrem 2.5 (see $[6,8])$. We set $\Psi=H_{\alpha}(1), 0<\alpha \leq 1$. Among all possible quadrature rules of type (2.25), the formula (2.26) is asymptotically optimal and has the error

$$
R_{N}[\Psi]=(1+o(1)) \frac{2^{1-\alpha}(1+\alpha)^{\alpha}}{\alpha^{1+\alpha} N^{\alpha}} .
$$

Proof. At the beginning we find value of $\zeta_{N}[\Psi]$. Taking into account the symmetry of the q.r. (2.25), we may restrict ourselves to the interval $[0,1]$.

In the segment $[0,1]$ we shall input a function

$$
\varphi^{*}(t)= \begin{cases}0, & 0 \leq t \leq t_{1}, \\ \min _{k}\left|t-t_{k}\right|, & t_{1} \leq t \leq 1,\end{cases}
$$

if $\alpha=1$ and

$$
\varphi^{*}(t)= \begin{cases}0, & 0 \leq t \leq t_{k}, k=\left[\frac{1+\alpha}{\alpha} 2^{2 / \alpha-2}\right]+1, \\ \min _{j}\left|t-t_{j}\right|^{\alpha}, & t_{k} \leq t \leq 1\end{cases}
$$

if $0<\alpha<1$.

We assume $M$ for $[\ln N]$ and divide the segment $[0,1]$ into smaller segments $\Delta_{k}=$ $\left[S_{k M}, S_{(k+1) M}\right], k=0,1, \ldots, l-1 ; \Delta_{l}=\left[S_{l M}, 1\right]$ where $S_{k M}=(k M / N)^{(1+\alpha) / \alpha}, k=0,1, \ldots, i$, $S_{(l+1) M}=1, l=[N / M]$. It is not difficult to see that

$$
\begin{aligned}
\int_{0}^{1} \frac{\varphi^{*}(\tau)}{\tau} d \tau & \geq \sum_{k=1}^{l+1} \frac{1}{S_{k M}} \int_{S_{(k-1) M}}^{S_{k M}} \varphi^{*}(\tau) d \tau \\
& \geq \frac{(1+\alpha)^{\alpha} M^{1+\alpha}}{2^{\alpha} \alpha^{1+\alpha} N^{1+\alpha}} \sum_{k=1}^{l+1}\left(\frac{k-\theta_{k}}{k}\right)^{(1+\alpha) / \alpha} \frac{1}{\left(n_{k-1}+1\right)^{\alpha}} \\
& \geq(1+o(1)) \frac{(1+\alpha)^{\alpha} M^{1+\alpha}}{2^{\alpha} \alpha^{1+\alpha} N^{1+\alpha}} \sum_{k=M}^{l} \frac{1}{\left(n_{k-1}+1\right)^{\alpha}}
\end{aligned}
$$


Here $0<\theta_{k}<1$ and $n_{k}$ is the number of nodes of q.r. (2.25) situated in the segment $\Delta_{k}$. While deriving relation (2.30) the inequality

$$
\min _{x_{1}, \ldots, x_{n}} \max _{\varphi \in H_{\alpha}(1)\left(x_{1}, \ldots, x_{n}\right)} \int_{a}^{b} \varphi(\tau) d \tau \geq \frac{(b-a)^{1+\alpha}}{(1+\alpha)(2(n+1))^{\alpha}}
$$

was used, where $H_{\alpha}(1)\left(x_{1}, \ldots, x_{n}\right)$ is the class of functions belonging to $H_{\alpha}(1)$ and vanishing at the nodes $a, x_{1}, \ldots, x_{n}, b$.

And then we will find the minimal value of the sum $\sum_{k=M}^{l}\left(n_{k-1}+1\right)^{-\alpha}$. We do not know the value of $\sum_{k=M}^{l} n_{k-1}$ but it is evident that the more the sum $\sum_{k=M}^{l} n_{k-1}$ the less the sum $\sum_{k=M}^{l}\left(n_{k-1}+1\right)^{-\alpha}$. That is why we will look for the minimum of the sum $V=\sum_{k=M}^{l}\left(n_{k-1}+1\right)^{-\alpha}$ if $\sum_{k=M}^{l} n_{k-1}=N$. Standard methods of mathematical analysis make it possible to find out that the nodes $n_{M-1}=n_{M}=\cdots=n_{l-1}=N /(l-M+1)$ give minimum of the sum $V$. Therefore $V \sim l^{1+\alpha} / N^{\alpha}$. Substituting this value into the expression (2.30) we conclude that for any nodes $t_{k}, 1 \leq k \leq N$ the inequality

$$
\sup \int_{0}^{1} \frac{\varphi(\tau)}{\tau} \geq(1+o(1)) \frac{(1+\alpha)^{\alpha}}{2^{\alpha} \alpha^{1+\alpha} N^{\alpha}}
$$

is valid, where the supremum is taken on all types of the functions $\varphi(\tau)$ belonging to class $H_{\alpha}(1)$ on the segment $[0,1]$ and vanishing at the points $0, t_{k}, 1 \leq k \leq N$. So,

$$
\zeta_{N}\left(H_{\alpha}(1)\right)=(1+o(1)) \frac{2^{1-\alpha}(1+\alpha)^{\alpha}}{\alpha^{1+\alpha} N^{\alpha}} .
$$

The lower bound is received.

We will estimate the error of the q.r. (2.27). It is easy to see that

$$
\left|R_{N}\right| \leq\left|\int_{t_{-1}}^{t_{1}} \frac{\varphi(\tau)}{\tau} d \tau-\int_{t_{-1}}^{t_{1}} \frac{\varphi(0)}{\tau} d \tau\right|+2 \sum_{k=1}^{N-1}\left|\int_{t_{k}}^{t_{k+1}} \frac{\varphi(\tau)-\varphi\left(t_{k}^{\prime}\right)}{\tau} d \tau\right|=r_{1}+r_{2}
$$

By estimating each expression $r_{1}, r_{2}$ separately

$$
\begin{aligned}
& r_{1}=2\left|\int_{0}^{t_{1}} \frac{\varphi(\tau)-\varphi(0)}{\tau} d \tau\right| \leq \frac{2 t_{1}^{\alpha}}{\alpha}=\frac{2}{N^{1+\alpha} \alpha}=o\left(\frac{1}{N^{\alpha}}\right) \\
& r_{2} \leq \frac{2^{1-\alpha} N^{(1+\alpha) / \alpha}}{1+\alpha} \sum_{k=1}^{N-1} \frac{\left(t_{k+1}-t_{k}\right)^{1+\alpha}}{k^{(1+\alpha) / \alpha}}=(1+o(1)) \frac{2^{1-\alpha}(1+\alpha)^{\alpha}}{\alpha^{1+\alpha} N^{\alpha}}
\end{aligned}
$$

and comparing the estimates (2.34), (2.35), and (2.36) with the estimate (2.32) we see that Theorem 2.5 is valid.

2.2.2. Integral on axis. In this section, we investigate calculation methods for the singular integrals

$$
J \varphi=\int_{-\infty}^{\infty} \frac{\varphi(\tau)}{\tau} d \tau
$$

on Hölder class of functions $H_{\alpha, \rho}(1)$, where $\rho(t)=(\max (1,|t|))^{\lambda}$. As a method of 
evaluating the integral (2.37) we use the quadrature rule

$$
J \varphi=\sum_{k=-N}^{N}{ }^{\prime} p_{k} \varphi\left(t_{k}\right)+R_{N},
$$

where $-A \leq t_{-N}<\cdots<t_{-1} \leq 0 \leq t_{1}<\cdots<t_{N} \leq A, A$ is a constant, which will be defined below the prime in the summation to indicate that $k \neq 0$. Input a quadrature rule

$$
\begin{aligned}
J \varphi= & \sum_{k=-N_{1}}^{N_{1}-1} " \varphi\left(t_{k}^{\prime}\right) \ln \left(\frac{t_{k+1}}{t_{k}}\right)-\frac{1}{\lambda} \sum_{k=M_{0}}^{M_{1}-1} \varphi\left(v_{k}^{\prime}\right)\left(\left(v_{k}\right)^{-\lambda}-\left(v_{k+1}\right)^{-\lambda}\right) \\
& -\frac{1}{\lambda} \sum_{k=M_{0}}^{M_{1}-1} \varphi\left(v_{-k}^{\prime}\right)\left(\left(v_{-k-1}\right)^{-\lambda}-\left(v_{-k}\right)^{-\lambda}\right)+R_{N}
\end{aligned}
$$

where

$$
\begin{aligned}
t_{ \pm k} & = \pm\left(\frac{k}{N_{1}}\right)^{(1+\alpha) / \alpha}, \quad t_{k}^{\prime}=\frac{\left(t_{k}+t_{k+1}\right)}{2}, \quad k=1,2, \ldots, N_{1}-1, \\
t_{-k}^{\prime} & =\frac{\left(t_{-k}+t_{-k+1}\right)}{2}, \quad k=2,3, \ldots, N_{1}, \\
v_{ \pm k} & = \pm\left(\frac{M_{1}}{k}\right)^{(1+\alpha) /(\lambda-\alpha)}, \quad k=M_{0}, M_{0}+1, \ldots, M_{1} ; M_{1}-M_{0}=N_{2}, \\
M_{0} & =\left[\frac{N_{2}}{\left(A^{(\lambda-\alpha) /(1+\alpha)}-1\right)}\right], \quad M_{1}=\left[\frac{A^{(\lambda-\alpha) /(1+\alpha)} N_{2}}{\left(A^{(\lambda-\alpha) /(1+\alpha)}-1\right)}\right], v \\
v_{ \pm k}^{\prime} & =\frac{\left(v_{ \pm k}+v_{ \pm k \pm 1}\right)}{2}, \quad k=M_{0}, M_{0}+1, \ldots, M_{1}-1, \\
N_{1} & =\left[N \frac{(\lambda-\alpha) A^{(\lambda-\alpha) /(1+\alpha)}}{\lambda A^{(\lambda-\alpha) /(1+\alpha)}-\alpha}\right], \quad N_{2}=\left[N \frac{\left(A^{(\lambda-\alpha) /(1+\alpha)}-1\right) \alpha}{\lambda A^{(\lambda-\alpha) /(1+\alpha)}-\alpha}\right], \quad N=N_{1}+N_{2},
\end{aligned}
$$

the double prime in the summation to indicate that $k \neq 0,-1$.

THEOREM 2.6 (see [13]). Set $\Psi=H_{\alpha, \rho}(1)$. Among all possible quadrature rules of the type (2.38), the formula (2.39) is asymptotically optimal and has the error $R_{N}[\Psi]=$ $L(N)$, where

$$
\begin{aligned}
& L(N)=2(1+o(1)) \\
& \times\left[\frac{\left(\lambda A^{(\lambda-\alpha) /(1+\alpha)}-\alpha\right)^{\alpha}}{N^{\alpha}}\left(\frac{C_{1}}{(\lambda-\alpha)^{\alpha} A^{\alpha(\lambda-\alpha) /(1+\alpha)}}+\frac{C_{2}}{\alpha^{\alpha}\left(A^{(\lambda-\alpha) /(1+\alpha)}-1\right)^{\alpha}}\right)\right. \\
& \left.+\frac{B(\alpha+1, \lambda-\alpha)}{A^{\lambda-\alpha}}\right] \\
& C_{1}=\frac{(1+\alpha)^{\alpha}}{2^{\alpha} \alpha^{1+\alpha}}, \quad C_{2}=\left(\frac{1+\alpha}{\lambda-\alpha}\right)^{1+\alpha} \frac{\left(A^{(\lambda-\alpha) /(1+\alpha)}-1\right)^{1+\alpha}}{A^{\lambda-\alpha} 2^{\alpha}(1+\alpha)}, \\
& A=\left(\frac{2 N \alpha(\lambda-\alpha)^{(\alpha+1) / \alpha}}{\lambda(1+\alpha)}(B(\alpha+1, \lambda-\alpha))^{1 / \alpha}+\frac{\alpha}{\lambda}\right)^{(1+\alpha) /(\lambda-\alpha)},
\end{aligned}
$$

and $B(\alpha, \lambda)$ is the beta-function. 
Proof. At the beginning find the lower bound of the value $\zeta_{N}[\Psi]$. Taking into account the symmetry of the q.r. (2.38), we restrict ourselves to the interval $[0, \infty)$. We set that the nodes of the q.r. (2.38) are situated on the segment $[0, A]$ and divide $[0, \infty)$ into three parts: $[0,1],[1, A],[A, \infty)$. Let $N_{1}$ be the number of the nodes $t_{k}$ of the q.r. (2.38) situated on the segment $[0,1], N_{2}$ is the number of the nodes $t_{k}$ of the q.r. (2.38) situated on the segment $[1, A]$. It is clear that $N_{1}+N_{2}=N$.

In Section 2.2.1 we constructed the asymptotically optimal q.r. (2.26) for calculating the integral (2.1).

Consider the integral

$$
\int_{1}^{A} \frac{f(\tau)}{\tau} d \tau
$$

Making use of the results in [2] we have

$$
\sup \int_{1}^{A} \frac{\varphi(\tau)}{\tau^{\lambda+1}} d \tau \geq(1+o(1))\left(\frac{1+\alpha}{\lambda-\alpha}\right)^{1+\alpha} \frac{\left(A^{(\lambda-\alpha) /(1+\alpha)}-1\right)^{1+\alpha}}{A^{\lambda-\alpha}} \frac{1}{2^{\alpha}(1+\alpha) N_{2}^{\alpha}},
$$

where the supremum is taken on all types of the functions $\varphi(t) \in H_{\alpha}(1)$ and being vanished at the points $t_{k}$, situated on the segment $[1, A]$. It will be seen below that for the optimal q.r. constant $A$ must be strived to infinity. So,

$$
\sup _{\varphi \in H_{\alpha}(1)} \int_{1}^{A} \frac{\varphi(\tau)}{\tau^{\lambda+1}} d \tau \geq(1+o(1)) C_{2} N_{2}^{-\alpha} .
$$

Using [38, Formula 24, page 298], we find that

$$
\sup \int_{A}^{\infty} \frac{\varphi(\tau)}{\tau^{\lambda+1}} d \tau \geq \int_{A}^{\infty} \frac{(\tau-A)^{\alpha}}{\tau^{\lambda+1}} d \tau=\int_{0}^{\infty} \frac{\tau^{\alpha}}{(\tau+A)^{\lambda+1}} d \tau=A^{\alpha-\lambda} B(\alpha+1, \lambda-\alpha),
$$

where $B(\alpha, \beta)=\Gamma(\alpha) \Gamma(\beta) / \Gamma(\alpha+\beta)$ is the beta-function, $\Gamma(\alpha)$ is the gamma-function, and the supremum is taken on all types of the functions $\varphi(\tau) \in H_{\alpha}(1)$ and vanishing at the nodes $t_{k}, 1 \leq k \leq N$.

We will find the distribution of the nodes $N_{1}$ and $N_{2}$ on the segments $[0,1]$ and $[1, A]$. For this purpose it is necessary to find the minimum of the function

$$
V\left(N_{1}, N_{2}\right)=C_{1} N_{1}^{-\alpha}+C_{2} N_{2}^{-\alpha}+A^{\alpha-\lambda} B(\alpha+1, \lambda-\alpha)
$$

under additional condition $N_{1}+N_{2}=N$.

In solving the problem on conditional extremum we find values of $N_{1}, N_{2}, A$ (see (2.40) and (2.41)) and receive the equality $\zeta_{N}[\Psi]=L(N)$.

The lower bound is received.

We will estimate the error of the q.r. (2.39). It is easy to see that

$$
\begin{aligned}
\left|R_{N}\right| \leq & \left|\int_{t_{-1}}^{t_{1}} \frac{\varphi(\tau)}{\tau} d \tau-\int_{t_{-1}}^{t_{1}} \frac{\varphi(0)}{\tau} d \tau\right|+2 \sum_{k=1}^{N_{1}-1}\left|\int_{t_{k}}^{t_{k+1}} \frac{\varphi(\tau)-\varphi\left(t_{k}^{\prime}\right)}{\tau} d \tau\right| \\
& +2 \sum_{k=M_{0}}^{M_{1}-1}\left|\int_{v_{k+1}}^{v_{k}} \frac{\varphi(\tau)-\varphi\left(v_{k}^{\prime}\right)}{\tau^{1+\lambda}} d \tau\right|+2\left|\int_{A}^{\infty} \frac{(\tau-A)^{\alpha}}{\tau^{1+\lambda}} d \tau\right| \leq L(N) .
\end{aligned}
$$

Comparing this estimate with the lower bound we see that Theorem 2.6 is valid. 
2.3. Asymptotically optimal quadrature rules for calculating singular integrals on the class $W_{\rho}^{r}(1)$

2.3.1. Finite segments. We will calculate the integral (2.1) with q.r.

$$
I \varphi=\sum_{k=-N}^{N} \sum_{l=0}^{\beta} p_{k l} \varphi^{(l)}\left(t_{k}\right)+R_{N}\left(\varphi, p_{k}, t_{k}\right)
$$

where $-1 \leq t_{-N}<\cdots<t_{-1}<t_{0}<t_{1}<\cdots<t_{N} \leq 1$.

For approximating a function $f(\tau)$ on the segment $\left[v_{k}, v_{k+1}\right]$ we will use the function $\tilde{f}\left(\tau,\left[v_{k}, v_{k+1}\right]\right)$, which was introduced in Section 1.3. Spline is received by combining the functions $\tilde{f}\left(\tau,\left[v_{k}, v_{k+1}\right]\right)$ and is denoted by $\tilde{f}(\tau)$.

THEOREM 2.7 (see [6, 8]). Among all types of the q.r. (2.48) on the class $W^{r}(1)$ with $\beta=r-1(r=1,2, \ldots)$ the quadrature rule

$$
\begin{aligned}
I \varphi= & \sum_{k=1}^{M-1}\left(\int_{t_{k}}^{t_{k+1}} \frac{\tilde{f}\left(\tau,\left[t_{k}, t_{k+1}\right]\right)}{\tau} d \tau+\int_{t_{-k-1}}^{t_{-k}} \frac{\tilde{f}\left(\tau,\left[t_{-k-1}, t_{-k}\right]\right)}{\tau} d \tau\right) \\
& +\sum_{k=1}^{r-1} \frac{f^{(k)}(0)}{k ! k} \frac{1}{N^{k(r+1) / r}}\left(1-(-1)^{k}\right)+R_{N},
\end{aligned}
$$

$t_{ \pm k}= \pm(k / N)^{(r+1) / r}, k=0,1, \ldots, N$ is asymptotically optimal. The error of the q.r. (2.49) is equal to

$$
R_{N}[\Psi]=(2+o(1))\left(\frac{r+1}{r}\right)^{r+1} \frac{1}{4^{r} r ! N^{r}} .
$$

Proof. We will first consider the lower bound. Taking into account the symmetry of the formula (2.48) it is sufficient to consider a gap $[0,1]$.

Consider an integral

$$
\int_{0}^{1} \frac{f(\tau)}{\tau} d \tau
$$

Now we use designations $S_{ \pm k}= \pm(k / N)^{(r+1) / r}(k=0,1, \ldots, N), M=[\ln N]$, $l=[N / M]$, Let $N_{k}$ be the number of nodes of the q.r. (2.48) on the segment $\Delta_{k}=$ $\left[S_{k}^{*}, S_{k+1}^{*}\right], k=0,1, \ldots, l$, where $S_{k}^{*}=S_{k M}, k=0,1, \ldots, l-1, S_{l+1}^{*}=1$ corresponding to the definition, $f^{+}(t)=(f(t)+|f(t)|) / 2, f^{-}(t)=(f(t)-|f(t)|) / 2$. To get the lower bound we can consider only the segment $[0,1]$. On this segment we will construct a function $f^{*}(t)$, equal to zero for $t \in\left[0, S_{M}\right]$, belonging to $W^{r}(1)$ and vanishing together with its derivatives up to $(r-1)$ order inclusive at the nodes $t_{k}(k=1,2, \ldots, N)$ of the q.r. (2.48) and the points $S_{k}^{*}(k=1,2, \ldots, l+1)$. Besides, we will require that

$$
\int_{S_{k}^{*}}^{S_{k+1}^{*}} f^{*}(\tau) d \tau \geq 0, \quad k=0,1, \ldots, l .
$$

It is obvious that

$$
\int_{0}^{1} \frac{f^{*}(\tau)}{\tau} d \tau \geq \sum_{k=1}^{l}\left[\left(\frac{1}{S_{k+1}^{*}}\right) \int_{S_{k}^{*}}^{S_{k+1}^{*}} f^{*}(\tau) d \tau+\left(\frac{1}{S_{k}^{*}}-\frac{1}{S_{k+1}^{*}}\right) \int_{S_{k}^{*}}^{S_{k+1}^{*}} f^{*-}(\tau) d \tau\right]=I_{1}+I_{2} .
$$


It is shown in [36] that at any position of the nodes $t_{k}$

$$
\inf _{p_{k l}} \sup _{\varphi \in W^{r}(1)}\left|\int_{0}^{1} \varphi(\tau) d \tau-\sum_{k=1}^{N} \sum_{l=0}^{r-1} p_{k l} \varphi\left(t_{k}\right)\right| \geq \frac{1}{r ![4(N-1)+2 \sqrt[r]{r+1}]^{r}} .
$$

According to Smolyk lemma and Nikol'skiĭ theorem [36] we have

$$
\begin{aligned}
& \sup _{\substack{\varphi \in W^{r}(1) ; \varphi^{(j)}\left(v_{i}\right)=0 ; \\
i=1,2, \ldots, N_{k}+2 ; j=0,1, \ldots, r-1}} \int_{S_{k}^{*}}^{S_{k+1}^{*}} \varphi(\tau) d \tau \\
& \geq\left(S_{k+1}^{*}-S_{k}^{*}\right)^{r+1} \inf _{p_{k l}, w_{k}} \sup _{\varphi \in W^{r}(1)}\left|\int_{0}^{1} \varphi(\tau) d \tau-\sum_{k=1}^{N_{k}+2} \sum_{l=0}^{r-1} P_{k l} \varphi^{(l)}\left(w_{k}\right)\right|,
\end{aligned}
$$

where $w_{i}$ is the set of the nodes of q.r. situated on the segment $\Delta_{k}$ and the points $S_{k}^{*}$, $S_{k+1}^{*}$. Therefore,

$$
\sup _{\varphi \in W^{r}(1) ; \varphi^{(j)}\left(v_{i}\right)=0} \int_{S_{k}^{*+1}}^{S_{k}^{*}} f(\tau) d \tau \geq \frac{\left(S_{k+1}^{*}-S_{k}^{*}\right)^{r+1}}{r !\left[4\left(N_{k}+1\right)+2 \sqrt[r]{r+1}\right]^{r}} .
$$

Then

$$
\begin{aligned}
I_{1} & =\sum_{k=1}^{l} \frac{1}{S_{k+1}^{*}} \int_{S_{k}^{*}}^{S_{k+1}^{*}} f^{*}(\tau) d \tau \\
& \geq \sum_{k=1}^{l} \frac{\left(S_{k+1}^{*}-S_{k}^{*}\right)^{r+1}}{S_{k+1}^{*} r !\left[4\left(N_{k}+1\right)+2 \sqrt[r]{r+1}\right]^{r}} \\
& \geq(1+o(1))\left(\frac{r+1}{r}\right)^{r+1} \frac{1}{r !}\left(\frac{M}{N}\right)^{r+1} \sum_{k=M_{1}}^{l} \frac{1}{\left[4\left(N_{k}+1\right)+2 \sqrt[r]{r+1}\right]^{r}} .
\end{aligned}
$$

We can find the distribution of the nodes $N_{k}$ minimizing the sum

$$
V=\sum_{k=M_{1}}^{l} \frac{1}{\left[4\left(N_{k}+1\right)+2 \sqrt[r]{r+1}\right]^{r}},
$$

provided that $\sum_{k=1}^{l} N_{k}=M$. This sum can only be reduced if we suppose that $N_{M_{1}}+$ $N_{M_{1}+1}+\cdots+N_{l}=M$. It is easy to verify that the sum $V$ reaches minimum, provided that $N_{M_{1}}=N_{M_{1}+1}=\cdots=N_{l}=N /(l-M+1)$ and this minimum is equal to $(1+o(1)) l^{r+1} /(4 N)^{r}$. So, it has been shown that the minimum is reached provided the values $N_{M_{1}}=\cdots=N_{l}=N /(l-M+1)$, which may be non-whole numbers. As we consider the problem of minimization of whole values, where the values $N_{M_{1}}, \ldots, N_{l}$ must be whole positive numbers, the minimum of the sum $V$ under these circumstances must not be less than $(1+o(1))\left(l^{r+1}\right) /\left((4 N)^{r}\right)$. Therefore,

$$
I_{1} \geq(1+o(1)) \frac{(r+1)^{r+1}}{4^{r} r^{r+1} r ! N^{r}} .
$$

Estimate the expression $I_{2}$

$$
I_{2} \leq \sum_{k=1}^{l} \frac{(r+1) M^{(r+1) / r}}{r(k+1) k^{(r+1) / r} N^{1+1 / r}} \int_{S_{k}^{*+1}}^{S_{k}^{*}} f^{*-}(\tau) d \tau .
$$


By construction every interval $\left[S_{k}, S_{k+1}\right]$ has at least one node where the function $f^{*}(t)$ with its derivatives up to order $(r-1)$ vanishes. In each interval we will take one node and denote it by $S_{k}^{* *}(k=1,2, \ldots, l)$. In the interval $\left[S_{k}, S_{k+1}\right]$ the function $f^{*}(t)$ may be represented as

$$
f^{*}(\tau)=\frac{1}{(r-1) !} \int_{S_{k}^{* *}}^{\tau}(\tau-t)^{r-1} f^{*(r)}(t) d t
$$

and therefore,

$$
\left|\int_{S_{k}}^{S_{k+1}} f^{*-}(\tau) d \tau\right| \leq \int_{S_{k}}^{S_{k+1}}\left|f^{*}(\tau)\right| d \tau \leq \frac{\left(S_{k+1}-S_{k}\right)^{r+1}}{(r+1) !} .
$$

So, from (2.60) and (2.62) we have that

$$
\left|I_{2}\right|=o\left(M^{-r}\right)
$$

Starting from that and the estimate of the sum $I_{1}$ we see that the upper bound of the estimate from below on the segment $[0,1]$ is not less than or equal to

$$
\zeta_{N}\left[W^{r}(1)\right] \geq(2+o(1)) \frac{((r+1) / r)^{r+1}}{4^{r} r ! M^{r}} .
$$

We can estimate the error of the q.r. (2.49)

$$
\begin{aligned}
\left|R_{N}\right| \leq & 2\left|\sum_{k=1}^{N-1} \int_{t_{k}}^{t_{k+1}} \frac{\varphi(\tau)-\tilde{\varphi}\left(\tau,\left[t_{k}, t_{k+1}\right]\right)}{\tau} d \tau\right| \\
& +\left|\int_{t_{-1}}^{t_{1}} \frac{\varphi(\tau)}{\tau} d \tau-\sum_{k=1}^{r-1} \frac{\varphi^{(k)}(0)}{k ! k} M^{-k(r+1) / r}\left(1-(-1)^{k}\right)\right|=r_{1}+r_{2} .
\end{aligned}
$$

It is easy to see that

$$
\begin{aligned}
r_{1} & =2\left|\sum_{k=1}^{N-1} \int_{t_{k}}^{t_{k+1}} \frac{f(\tau)-\tilde{f}\left(\tau,\left[t_{k}, t_{k+1}\right]\right)}{\tau} d \tau\right| \\
& =2\left|\sum_{k=1}^{N-1} \int_{t_{k}}^{t_{k+1}} \frac{1}{\tau} \int_{t_{k}}^{t_{k+1}}\left(\frac{K_{r}(\tau-t)}{(r-1) !}-\sum_{j=0}^{r-1} \frac{B_{k j}}{(r-1-j) !} K_{r-j}\left(t_{k+1}-t_{k}\right)\right) f^{(r)}(t) d t d \tau\right| \\
& =2\left|\sum_{k=1}^{N-1} \int_{t_{k}}^{t_{k+1}} f^{(r)}(t) \int_{t_{k}}^{t_{k+1}} \frac{1}{\tau}\left(\frac{K_{r}(\tau-t)}{(r-1) !}-\sum_{j=0}^{r-1} \frac{B_{k j}}{(r-1-j) !} K_{r-j}\left(t_{k+1}-t\right)\right) d \tau d t\right| \\
& \leq 2 \sum_{k=1}^{N-1} \frac{1}{t_{k}} \int_{t_{k}}^{t_{k+1}}\left|f^{(r)}(t)\right|\left|\frac{\left(t_{k+1}-t\right)^{r}}{r !}-\sum_{j=0}^{r-1} \frac{B_{k j}\left(t_{k+1}-t_{k}\right)}{(r-1-j) !}\left(t_{k+1}-t\right)^{r-j-1}\right| d t \\
& \leq \frac{2}{r !} \sum_{k=1}^{M-1} \frac{1}{t_{k}} \int_{t_{k}}^{t_{k+1}}\left|\left(t_{k+1}-t\right)^{r}-\sum_{j=0}^{r-1} \frac{r ! B_{k j}\left(t_{k+1}-t_{k}\right)}{(r-1-j) !}\left(t_{k+1}-t\right)^{r-j-1}\right| d t .
\end{aligned}
$$


It is known that (Nikol'skiĭ [36])

$$
\int_{a-h}^{a+h}\left|R_{r q}(a, h, x)\right|^{q} d x=\frac{2 h^{r q+1}\left[R_{r q}(1)\right]^{q}}{r q+1} .
$$

Then

$$
\begin{aligned}
& r_{1} \leq 4 R_{r 1}(1) \sum_{k=1}^{N-1} \frac{\left(\left(t_{k+1}-t_{k}\right) / 2\right)^{r+1}}{t_{k}(r+1) !} \leq(1+o(1)) \frac{(r+1)^{r+1}}{2^{2 r-1} r^{r+1} r ! N^{r}}, \\
& r_{2}=\frac{1}{(r+1) !}\left|\int_{t_{-1}}^{t_{1}} \tau^{-1}\left(\int_{0}^{\tau}(\tau-t)^{r-1} f^{(r)}(t) d t\right) d \tau\right| \leq \frac{2 M^{-r-1}}{r ! r}
\end{aligned}
$$

From the estimations (2.65) and (2.68) we get

$$
R_{N}[\Psi] \leq(1+o(1)) \frac{(r+1)^{r+1}}{2^{2 r-1} r^{r+1} r ! N^{r}} .
$$

Comparing this estimate with the estimate (2.64) we see that Theorem 2.7 is valid. Let $M=\left[\ln ^{1 / 2 r} N\right], L=[N / M]$. Consider a quadrature rule

$$
\begin{aligned}
I f= & \sum_{k=1}^{r-1} D_{n, r, p}\left(f^{(k)}(0)\right) \frac{1-(-1)^{k}}{k ! k} t_{1}^{*} \\
+ & \sum_{k=1}^{L-1}\left[\int_{t_{k}^{*}}^{t_{k+1}^{*}} D_{n, r, p}\left(T_{r-1}\left(f,\left[t_{k}^{*}, t_{k+1}^{*}\right]\right), t_{k}^{*}\right)\left(\frac{1}{\tau}-\frac{1}{t_{k+1}^{*}}\right) d \tau\right. \\
& \left.\quad+\int_{t_{-k-1}^{*}}^{t_{-k}^{*}} D_{n, r, p}\left(T_{r-1}\left(f ;\left[t_{-k-1}^{*}, t_{-k}^{*}\right]\right) t_{-k}^{*}\right)\left(\frac{1}{\tau}-\frac{1}{t_{-k-1}^{*}}\right) d \tau\right] \\
& +\sum_{k=0}^{L-1}\left(l_{M, r, p}\left(f,\left[t_{k}^{*}, t_{k+1}^{*}\right]\right) t_{k+1}^{*-1}+l_{M, r, p}\left(f,\left[t_{-k-1}^{*}, t_{-k}^{*}\right]\right) t_{-k-1}^{*-1}\right)+R_{N},
\end{aligned}
$$

where $t_{ \pm k}^{*}= \pm(k / L)^{v}, k=0,1, \ldots, L, v=(r q+1) /(r q+1-q), 1 / p+1 / q=1$. The operators $D_{n, r, p}$ and $T_{r-1}\left(f, \Delta_{k}, c_{k}\right)$ were introduced in Section 1.3.

THEOREM 2.8 (see [8]). Let $\Psi=W_{p}^{r}(1,1 ;[-1,1]), 1<p \leq \infty, r=1,2, \ldots$. Among all possible quadrature rules of the type (2.48), the formula (2.70) is asymptotically optimal and has the error

$$
R_{N}(\Psi)=(1+o(1)) \frac{1}{N^{r}}\left(\frac{r q+1}{r q+1-q}\right)^{r+1 / q} \inf _{c}\left\|D_{r}(t)-c\right\|_{L_{q}[0,1]} .
$$

2.3.2. Integrals on axis. In this section, we investigate the calculation methods for singular integrals (2.37) on the class of functions $W_{\rho}^{r}(1)$, where $\rho_{0}(t)=\max (1,|t|)$, $\rho_{1}(t)=\left(\rho_{0}(t)\right)^{\lambda}$.

We will calculate the integral (2.37) with q.r.

$$
J \varphi=\sum_{k=-N}^{N} \sum_{l=0}^{\beta} p_{k l} \varphi^{(l)}\left(t_{k}\right)
$$

where $-A \leq t_{-N}<\cdots<t_{-1}<t_{0}<t_{1}<\cdots<t_{N} \leq A$, constant $A$ will be defined below. 
TheOrem 2.9 (see [13]). Among all types of the q.r. (2.72) where $\beta=r-1(r=$ $1,2, \ldots)$ on the class $\Psi=W_{\rho}^{r}(1)$, the quadrature rule

$$
\begin{aligned}
J \varphi= & \sum_{k=1}^{M-1}\left(\int_{t_{k}}^{t_{k+1}} \frac{\tilde{f}\left(\tau,\left[t_{k}, t_{k+1}\right]\right)}{\tau} d \tau+\int_{t_{-k-1}}^{t_{-k}} \frac{\tilde{f}\left(\tau,\left[t_{-k-1}, t_{-k}\right]\right)}{\tau} d \tau\right) \\
& +\sum_{k=1}^{r-1} \frac{f^{(k)}(0)}{k ! k} M^{-k(r+1) / r}\left(1-(-1)^{k}\right) \\
& +\sum_{k=M_{0}}^{M_{1}-1}\left(\int_{v_{k+1}}^{v_{k}} \frac{\tilde{f}\left(\tau,\left[v_{k+1}, v_{k}\right]\right)}{\tau} d \tau+\int_{v_{-k}}^{v_{-k-1}} \frac{\tilde{f}\left(\tau,\left[v_{-k}, v_{-k-1}\right]\right)}{\tau} d \tau\right)+R_{N},
\end{aligned}
$$

where

$$
\begin{aligned}
t_{ \pm k} & = \pm\left(\frac{k}{M}\right)^{(r+1) / r}, \quad k=0,1, \ldots, M \\
v_{ \pm k} & = \pm\left(\frac{M_{1}}{k}\right)^{(r+1) /(\lambda-r)}, \quad k=M_{0}, M_{0}+1, \ldots, M_{1}, \\
M_{0} & =\left[\frac{n}{A^{(\lambda-r) /(r+1)}-1}\right], \quad M_{1}=\left[n A^{(\lambda-r) /(r+1)} A^{(\lambda-r) /(r+1)}-1\right], \\
n & =\left[\frac{r\left(A^{(\lambda-r) /(r+1)}-1\right)}{\lambda A^{(\lambda-r) /(r+1)}-r} N\right], \quad M=\left[\frac{A^{(\lambda-r) /(r+1)}(\lambda-r)}{\lambda A^{(\lambda-r) /(r+1)}-r} N\right], \\
A & =\left(\frac{4 N r(\lambda-r)}{\lambda(r+1)^{(r+1) / r}}(D(r, \lambda))^{1 / r}+\frac{r}{\lambda}\right)^{(r+1) /(\lambda-r)},
\end{aligned}
$$

is asymptotically optimal. The error of the q.r. (2.73) is equal to

$$
R_{N}[\Psi]=(1+o(1)) \frac{(r+1)^{r+1}}{2^{2 r-1} r^{r} r !(\lambda-r)^{r}}\left(\frac{C_{1}(r+1)}{C_{2} r}+\frac{\lambda^{r}}{\lambda-r}\right) \frac{1}{N^{r}},
$$

where

$$
C_{1}=\frac{D(r, \lambda)}{(\lambda-r) r !}, \quad C_{2}=\frac{(r+1)^{r+1}}{(\lambda-r)^{r+1} 4^{r} r^{r} r !}, \quad D(r, \lambda)=\sum_{k=0}^{r} \frac{r !(-1)^{k}(\lambda-r)}{k !(r-k) !(\lambda-r+k)} .
$$

Proof of this theorem is the union of the proofs of Theorems 2.6 and 2.9 but the technicality is more complex.

2.4. Optimal with respect to order quadrature rules. The optimal with respect to order quadrature rules can be useful in practical calculations which have good numerical properties and which are simpler for program realization than asymptotically optimal quadrature rules.

Consider the singular integrals as (2.1). We will compute the integrals $I f$ by quadrature rules as

$$
I f=\sum_{k=-N}^{N} \sum_{l=0}^{\rho} p_{k l} f^{(l)}\left(t_{k}\right)+R_{N}\left(f, p_{k l} \cdot t_{k}\right),
$$

where $-1 \leq t_{-N}<\cdots<t_{-1} \leq 0 \leq t_{1}<\cdots<t_{N} \leq 1$. 
THEOREM 2.10 (see [6]). Let $\Psi=W^{r}(1), r=2,4, \ldots$. Let the integral If be calculated by the quadrature rule of the type (2.77), where $\rho=0$. Then

$$
\zeta_{N}(\Psi) \geq(1+o(1)) K_{r} \frac{(r+1)^{r+1}}{2^{r-1} \pi^{r} r^{r} N^{r}}
$$

where $K_{r}$ is Favar constant.

Let $N_{1}=[N / r]+1$. Let $\Delta_{K}=\left[S_{k}, S_{k+1}\right], k=0,1, \ldots, N_{1}-1, \Delta_{-k}=\left[S_{-k-1}, S_{k}\right], k=$ $0,1, \ldots, N_{1}-1$, where $S_{ \pm k}= \pm(r k / N)^{(r+1) / r}, k=0,1, \ldots, N_{1}-1$. Define $S_{ \pm N_{1}}$ as $S_{ \pm N_{1}}=$ \pm 1 . Let $\zeta_{k}, k=1,2, \ldots, r$, be the nodes of Chebyshev polynomial of the type I.

Let $\zeta_{k}^{\prime}, k=1,2, \ldots, l$, be the result of mapping the segment $[-1,1]$ onto the segment $[a, b]$. Let $P_{r}(f,[a, b])$ be the Lagrange interpolated polynomial: $P_{r}(f,[a, b])\left(\zeta_{k}^{\prime}\right)=$ $f\left(\zeta_{k}^{\prime}\right), k=1,2, \ldots, r$.

Then the integral $I f$ we will be calculated by the quadrature rule

$$
\begin{aligned}
\int_{-1}^{1} \frac{f(\tau)}{\tau} d \tau= & \sum_{k=1}^{N_{1}-1} \int_{S_{k}}^{S_{k+1}} \frac{P_{r}\left(\tau, \Delta_{k}\right)}{\tau} d \tau+\int_{S_{-1}}^{S_{1}} \frac{P_{2 r}\left(\tau,\left[S_{-1}, S_{1}\right]\right)}{\tau} d \tau \\
& +\sum_{k=1}^{N_{1}-1} \int_{S_{-k-1}}^{S_{-k}} \frac{P_{k}\left(\tau, \Delta_{-k}\right)}{\tau} d \tau+R_{N} .
\end{aligned}
$$

THEOREM 2.11 (see [6]). Let $\Psi=W^{r}(1), r=1,2, \ldots$. Among all possible quadrature rules of the type (2.77), where $\rho=0$, the quadrature rule (2.79) is optimal with respect to order and its error occurs

$$
R_{N}(\Psi)=(1+o(1)) \frac{(r+1)^{r+1}}{r ! r 2^{2 r-1} N^{r}} .
$$

Consider a quadrature rule

$$
\begin{aligned}
\int_{-1}^{1} \frac{f(\tau)}{\tau} d \tau= & \sum_{k=1}^{N-1}\left[\int_{S_{k}}^{S_{k+1}} \frac{T_{r-1}\left(f, \Delta_{k}, S_{k}\right)}{\tau} d \tau+\int_{S_{-k-1}}^{S-k} \frac{T_{r-1}\left(f, \Delta_{-k}, S_{-k}\right)}{\tau} d \tau\right] \\
& +\sum_{k=1}^{r-1} f^{(k)}(0) \frac{1-(-1)^{k}}{N^{(r+1) k / r} k ! k}+R_{N},
\end{aligned}
$$

where $\Delta_{k}=\left[S_{k}, S_{k+1}\right], \Delta_{-k}=\left[S_{-k-1}, S_{-k}\right], k=0,1, \ldots, N-1, S_{ \pm k}= \pm(k / N)^{(r+1) / r}, k=$ $0,1, \ldots, N$.

THEOREM 2.12 (see [6]). Let $\Psi=W^{r}(1), r=1,2, \ldots$ Among all possible quadrature rules of the type (2.77), where $\rho=r-1$, the quadrature rule (2.81) is optimal with respect to order and its error holds

$$
R_{N}(\Psi) \leq \frac{(r+1)^{r+1}}{r^{r+1} 2^{r-1}(r+1) ! N^{r}} .
$$

We give now the description of the quadrature rule which is not optimal with respect to order but make use of one operation of multiplication and $A N \ln N$ operation of addition. 
Consider a quadrature rule

$$
I f=\frac{1}{N} \sum_{k=-M, k \neq 0}^{M} f\left(t_{k}^{1}\right)+R_{N}
$$

where $t_{ \pm k}= \pm N^{-1 / \alpha} e^{k / N}, t_{k}^{1}=\left(t_{k+1}+t_{k}\right) / 2, t_{-k}^{1}=\left(t_{-k-1}+t_{-k}\right) / 2, k=1,2, \ldots, M, M=$ $\left[\alpha^{-1} N \ln N\right]$.

THEOREM 2.13 (see [6]). Let $\Psi=H_{\alpha}(1), 0<\alpha \leq 1$. The quadrature rule (2.83) has the error $R_{N}[\Psi] \leq 2\left(e^{\alpha / N} /(\alpha N)+1 /\left(2^{\alpha} \alpha N^{\alpha}\right)+1 /\left(\alpha N^{1+\alpha}\right)\right)$.

Proofs of Theorems 2.10, 2.11, 2.12, and 2.13 are given in [6]. At the end of the section we describe one optimal with respect to order quadrature rule that is very simple in realization. Convergence of this quadrature rule was proved in [12]. Introduce the nodes $t_{ \pm k}= \pm(k / N)^{v}, k=0,1, \ldots, M, v=(r+1) / r$. Let $\zeta_{1}, \ldots, \zeta_{r} \in[-1,1]$ be the nodes of order $r$ Legendre polynomial. On every subinterval $\left[t_{k}, t_{k+1}\right], k=$ $-n, \ldots, N-1$, we construct interpolated polynomial $P_{r}\left(f,\left[t_{k}, t_{k+1}\right]\right)$ of degree $r-1$ with nodes $t_{k}+\left(1+\zeta_{v}\right)\left(t_{k+1}-t_{k}\right) / 2, v=1,2, \ldots, r$. The integral (2.1) is approximated by the quadrature rule

$$
\begin{aligned}
I \varphi= & \sum_{k=1}^{N-1}\left(\int_{t_{-k-1}}^{t_{-k}} P_{r}\left(\frac{\varphi(\tau)}{\tau},\left[t_{-k-1}, t_{-k}\right]\right) d \tau+\int_{t_{k}}^{t_{k+1}} P_{r}\left(\frac{\varphi(\tau)}{\tau},\left[t_{k}, t_{k+1}\right]\right) d \tau\right) \\
& +\int_{t_{-1}}^{t_{1}} P_{r}\left(\frac{\varphi(\tau)-\varphi(0)}{\tau}\right) d \tau+R_{N} .
\end{aligned}
$$

Its error is equal to

$$
R_{N}=(1+o(1)) \frac{2^{2 r+1} r !(r+1)^{r+1}}{(2 r) ! r^{r+1} N^{r}}
$$

REMARK 2.14. The quadrature rule (2.84) is asymptotically optimal for $r=1$ and optimal with respect to order for $r=2,3, \ldots$.

2.5. Stability of quadrature rules. Consider singular integrals of the kind (2.1), which we will compute by quadrature rule as (2.2).

Practically, we cannot calculate the exact values of the functionals $f\left(t_{k}\right)$ and weights $p_{k}$. It is necessary to investigate the influence of the calculation error of the functionals $f\left(t_{k}\right)$ and the weights $p_{k}$ on exactness of quadrature rules.

Let the functionals be calculated with exactness $\epsilon=\left|f\left(t_{k}\right)-\bar{f}\left(t_{k}\right)\right| \leq \epsilon, k=-N, \ldots$, $-1,0,1, \ldots, N$.

THEOREM 2.15 (see [6]). Let $\Psi=H_{\alpha}(1), 0<\alpha \leq 1$. Let the functionals $f\left(t_{k}\right)$ be calculated with the error equal to $\epsilon, 0<\epsilon<1$. In this case for any points $t_{k} \in[-1,1]$, $k=-N, \ldots,-1,0,1, \ldots, N$ the error of quadrature rules of the type (2.2) is not less than $(1+o(1))\left(1-\epsilon^{1 /(1-\alpha)}\right)^{1+\alpha} 2^{1-\alpha}(1+\alpha)^{\alpha} /\left(\alpha^{1+\alpha} N^{\alpha}\right)+2 \epsilon(|\ln \epsilon|+1) / \alpha$. 
TheOrem 2.16 (see [6]). Let $\Psi=H_{\alpha}(1), 0<\alpha \leq 1$. Let $\left|f\left(t_{k}\right)-\bar{f}\left(t_{k}\right)\right| \leq \epsilon, k=$ $-N, \ldots,-1,0,1, \ldots, N$. Then the quadrature rule

$$
I f=\sum_{k=-N, k \neq-1,0}^{N-1} \bar{f}\left(t_{k}^{\prime}\right) \ln \frac{t_{k+1}}{t_{k}}+R_{N},
$$

where $t_{ \pm 1}= \pm(k / N)^{(1+\alpha) / \alpha}, k=0,1, \ldots, N, t_{ \pm k}^{\prime}=\left(t_{ \pm k}+t_{ \pm k+1}\right) / 2$ has the error $\left|R_{N}\right| \leq$ $(1+o(1)) 2^{1-\alpha}(1+\alpha)^{\alpha} /\left(\alpha^{1+\alpha} N^{\alpha}\right)+2 \epsilon \alpha^{-1}(1+\alpha) \ln N+A N^{-1-\alpha}$.

THEOREM 2.17 (see [6]). Let $\Psi=H_{\alpha}, 0<\alpha \leq 1$. Let $\left|f\left(t_{k}\right)-\bar{f}\left(t_{k}\right)\right| \leq \epsilon, k=-N, \ldots$, $-1,0,1, \ldots, N$. Then the quadrature rule

$$
I f=\sum_{k=-N}^{N-1}, \bar{f}\left(t_{k}^{\prime}\right) \frac{t_{k+1}}{t_{k}}+R_{N},
$$

where $t_{ \pm k}= \pm(k / N)^{(1+\alpha) / \alpha} ; t_{ \pm k}^{\prime}=\left(t_{ \pm k}+t_{ \pm k+1}\right) / 2$; the prime in the summation indicates that $k \notin\left[-k^{*}, k^{*}\right], k^{*}=\left[N \epsilon^{1 /(1+\alpha)}\right]$ has the error $\left|R_{N}\right| \leq 2 \epsilon(|\ln \epsilon|+1) / \alpha+2^{1-\alpha}(1+$ $\alpha)^{\alpha}\left(1+(1+\alpha) N^{-1} \ln N\right) /\left(\alpha^{1+\alpha} N^{\alpha}\right)$.

\section{Optimal methods of calculating singular integrals with Cauchy and Hilbert kernels}

3.1. Introduction. In this section, we will investigate optimal methods of the calculation of singular integrals with Cauchy and Hilbert kernels. Consider singular integrals with Hilbert kernel as

$$
F \phi=\frac{1}{2 \pi} \int_{0}^{2 \pi} \phi(\sigma) \operatorname{ctg} \frac{\sigma-s}{2} d \sigma, \quad s \in[0,2 \pi]
$$

that we will compute by quadrature rules as

$$
F \phi=\sum_{k=1}^{N} \sum_{l=0}^{\rho} p_{k l}(s) \phi^{l}\left(s_{k}\right)+R_{N}\left(s, s_{k}, p_{k}(s), \phi\right)
$$

with nodes $0 \leq s_{k} \leq 2 \pi$ and weights $p_{k}(s), k=1,2, \ldots, N$, and singular integrals with Cauchy kernel as

$$
K \phi=\int_{-1}^{1} \frac{\phi(\tau) d \tau}{\tau-t}, \quad(t \in(-1,1)),
$$

that we will compute by quadrature rules as

$$
K \phi=\sum_{k=-N}^{N} \sum_{l=0}^{\rho} p_{k l}(t) \phi^{l}\left(t_{k}\right)+R_{N}\left(t, t_{k}, p_{k l}(s), \phi\right),
$$

where $-1 \leq t_{-N}<\cdots<t_{-1}<t_{0}<t_{1}<\cdots<t_{N} \leq 1$. 


\subsection{Asymptotically optimal quadrature rules on the class $H_{\alpha}(1)$}

THEOREM 3.1 (see $[6,8])$. Let $\Psi=H_{\alpha}(1)(0<\alpha \leq 1)$. Then among all possible types of the quadrature rules as (3.2), where $\rho=0$, the formula

$$
\begin{aligned}
F \phi= & \frac{1}{2 \pi} \sum_{k=1, k \neq v, v \pm 1}^{N} \int_{t_{k-1}}^{t_{k}} \phi\left(t_{k-1}^{\prime}\right) \operatorname{ctg} \frac{\sigma-s}{2} d \sigma \\
& +\frac{1}{2 \pi} \int_{t_{v-2}}^{t_{v+1}} \phi\left(t_{v-1}^{\prime}\right) \operatorname{ctg} \frac{\sigma-s}{2} d \sigma+R_{N}(s)
\end{aligned}
$$

is asymptotically optimal. There is $t_{k}=2 k \pi / N, t_{k}^{\prime}=(2 k+1) \pi / N, k=0,1, \ldots, N, s \in$ $\left[t_{v-1}, t_{v}\right)$. The quadrature rule (3.5) error is equal to

$$
R_{N}[M]=2 \ln N / \pi^{1-\alpha}(1+\alpha) N^{\alpha}+0\left(N^{-\alpha}\right) .
$$

TheOrem 3.2 (see $[6,8])$. Let $\Psi=H_{\omega}$, where $\omega(\sigma) \in \Phi$. Then among all possible quadrature rules using $N$ values of integrand function the formula (3.5) is asymptotically optimal. The error of this formula is equal to

$$
R_{N}[\Psi]=2 N(\ln N+0(1)) \frac{1}{\pi^{2}} \int_{0}^{\pi / N} \omega(\sigma) d \sigma .
$$

THEOREM 3.3 (see [8]). Let $\Psi=H_{\alpha}(1)(0<\alpha \leq 1)$. Then among all possible quadrature rules as (3.4), where $\rho=0$, the formula

$$
\mathbf{T} \phi=R_{N}+ \begin{cases}\phi\left(t_{0}\right) \int_{-1}^{t_{2}} \frac{d \tau}{\tau-t}+\sum_{k=2}^{2 N-1} \phi\left(t_{k}^{\prime}\right) \int_{t_{k}}^{t_{k+1}} \frac{d \tau}{\tau-t}, & t_{0}<t \leq t_{1} \\ \sum_{k=0}^{2 N-1} \phi\left(t_{k}^{\prime}\right)^{\prime} \int_{t_{k}}^{t_{k+1}} \frac{d \tau}{\tau-t}+\phi\left(t_{j}^{\prime}\right) \int_{t_{j-1}}^{t_{j+2}} \frac{d \tau}{\tau-t}, & t_{1}<t \leq t_{2 N-1} \\ \sum_{k=0}^{2 N-2} \phi\left(t_{k}^{\prime}\right) \int_{t_{k}}^{t_{k+1}} \frac{d \tau}{\tau-t}+\phi\left(t_{2 N}\right) \int_{t_{2 N-1}}^{t_{2 N}} \frac{d \tau}{\tau-t}, & t_{2 N-1} \leq t<t_{2 N}\end{cases}
$$

where $_{k}=-1+k / N, k=0,1, \ldots, 2 N, t_{k}^{\prime}=\left(t_{k}+t_{k+1}\right) / 2, k=0,1, \ldots, 2 N-1, t \in\left[t_{j}, t_{j+1}\right)$, the prime in the summation indicate that $k \neq j-1, j, j+1$, is asymptotically optimal. The error $R_{N}[\Psi]=(1+o(1)) 2^{1-\alpha} \ln N /(1+\alpha) N^{\alpha}$ is valid.

REMARK 3.4. Theorem 3.3 is valid for even number of the nodes too.

THEOREM 3.5 (see $[6,8])$. Let $\phi \in H_{\omega}(\omega \in \Phi)$. Then among all possible quadrature rules as (3.4), where $\rho=0$, the formula (3.8) is asymptotically optimal. The error of this formula holds

$$
R_{N}\left[H_{\omega}\right]=(1+o(1)) 2(2 N+1) \int_{0}^{1 /(2 N+1)} \omega(t) d t \ln N .
$$

REMARK 3.6. Theorem 3.5 is valid for even number of the nodes too. 
Proof of Theorem 3.1. At first let us find the lower bound of value $\zeta_{N}\left[H_{\alpha}(1)\right]$ in computing the integral $F \phi$ by the quadrature rule (3.2), where $\rho=0$. Let $S$ be a vector $S=\left(s_{1}, \ldots, s_{N}\right)$ of the nodes of the quadrature rule (3.2). Fix an arbitrary value $s_{j}$. Let the point $s_{j}+\pi$ belongs to the segment $\bar{\triangle}_{j}=\left[s_{\bar{j}}, s_{\bar{j}+1}\right]$, the endpoints of which are neighboring nodes of the vector $S$.

We associate to each value $s_{j}$ the $2 \pi$ periodic function defined for $\alpha=1$ by expression

$$
\phi_{\mathbf{j}}^{*}(\sigma)= \begin{cases}0, & \sigma \in \bar{\Delta}_{j} ; \\ \min _{k}\left(\left|\sigma-s_{k}\right|\right) \operatorname{sgnctg} \frac{\sigma-s_{j}}{2}, & \sigma \in[0,2 \pi] \backslash \overline{\Delta_{j}} ;\end{cases}
$$

and for $0<\alpha<1$ defined by expression

$$
\phi_{\mathbf{j}}^{*}(\sigma)= \begin{cases}0, & \sigma \in \Omega_{j}=\left[s_{j-v}, s_{j+1+v}\right] \cup\left[s_{\bar{j}-v}, s_{\bar{j}+v}\right] ; \\ \min _{k}\left(\left|\sigma-s_{k}\right|\right) \operatorname{sgnctg} \frac{\sigma-s_{j}}{2}, & \sigma \in[0,2 \pi] \backslash \Omega_{j} .\end{cases}
$$

Here $v=\left[2^{1 / \alpha-2}\right]+1, j=1,2, \ldots, N$, moreover, $s_{N+1}=2 \pi+s_{1}$. Similarly $s_{N+r}=2 \pi+s_{r}$, $s_{-q}=s_{N-q}-2 \pi$ for $r>0, q>0$. Introduce a designation $x_{k}=\left(s_{k+1}-s_{k}\right) / 2$. There arises two cases:

(1) at least one of the values $x_{v}(v=1,2, \ldots, N)$ is not less than $2 \pi N^{-1}(\ln N)^{1 / \alpha}$;

(2) all of these values are separately less than $2 \pi N^{-1}(\ln N)^{1 / \alpha}$.

In the first case assuming, without loss of generality, that $2 \pi N^{-1}(\ln N)^{1 / \alpha} \leq x_{1} \leq \pi / 2$ we have

$$
\max _{0 \leq s \leq 2 \pi} \frac{1}{2 \pi} \int_{0}^{2 \pi} \phi_{1}^{*}(\sigma) \operatorname{ctg} \frac{\sigma-s}{2} d \sigma \geq \frac{2 \pi^{\alpha-1} \ln N}{(1+\alpha) N^{\alpha}} .
$$

As for the second case, divide a segment $[0,2 \pi]$ into $M=\left[N / 2 \ln ^{1 / \alpha} N\right]$ equal portions by the points $v_{k}=2 k \pi / M, k=0,1, \ldots, M$. Then a single node of the q.r. (3.2) exists at least in each segment $\left[v_{k}, v_{k+1}\right]$. Choose in each segment $\left[v_{k}, v_{k+1}\right]$ exactly one single node that we denote by $s_{k}^{*}(k=1,2, \ldots, M)$. Fix an arbitrary value $1 \leq j \leq M$ and estimate the integral

$$
\begin{aligned}
\left(F \phi_{j}^{*}\right)\left(s_{j}^{*}\right) & =\frac{1}{2 \pi} \int_{0}^{2 \pi} \phi_{j}^{*}(\sigma) \operatorname{ctg} \frac{\sigma-s_{j}^{*}}{2} d \sigma \\
& \geq \frac{1}{2 \pi} \sum_{k=1}^{[M / 2]-2} \frac{M}{(k+1) \pi}\left(\int_{v_{j+k}}^{v_{j+k+1}} \phi^{*}(\sigma) d \sigma+\int_{v_{j-k}}^{v_{j-k+1}} \phi^{*}(\sigma) d \sigma\right),
\end{aligned}
$$

where $\phi^{*}(\sigma)$ is $2 \pi$ periodic function defined by formula $\phi^{*}(\sigma)=\min _{k}\left(\left|\sigma-s_{k}\right|^{\alpha}\right)$. Having averaged values $\left(F \phi_{j}^{*}\right)\left(s_{j}^{*}\right)$, we have

$$
\begin{aligned}
\max _{s, j}\left(F \phi_{j}^{*}\right)(s) & \geq \frac{1}{M} \sum_{j=1}^{M} \frac{M}{2 \pi^{2}} \sum_{k=1}^{[M / 2]-2} \frac{1}{k+1}\left(\int_{v_{j+k}}^{v_{j+k+1}} \phi^{*}(\sigma) d \sigma+\int_{v_{j-k}}^{v_{j-k+1}} \phi^{*}(\sigma) d \sigma\right) \\
& =\frac{1}{2 \pi^{2}} \sum_{k=1}^{[M / 2]-2} \frac{1}{k+1} \sum_{j=1}^{M}\left(\int_{v_{j+k}}^{v_{j+k+1}} \phi^{*}(\sigma) d \sigma+\int_{v_{j-k}}^{v_{j-k+1}} \phi^{*}(\sigma) d \sigma\right) \\
& =\frac{1}{\pi^{2}} \int_{0}^{2 \pi} \phi^{*}(\sigma) d \sigma \sum_{k=1}^{[M / 2]-2} \frac{1}{k+1} \sim \frac{\ln N}{\pi^{2}} \int_{0}^{2 \pi} \phi^{*}(\sigma) d \sigma .
\end{aligned}
$$


Thus this problem has reduced itself to finding the lower bound of the values $\int_{0}^{2 \pi} \phi^{*}(\sigma) d \sigma$ in varying the nodes $s_{k}(k=1,2, \ldots, N)$. It is obvious that

$$
\int_{0}^{2 \pi} \phi^{*}(\sigma) d \sigma=\frac{2}{1+\alpha} \sum_{k=0}^{N-1} x_{k}^{1+\alpha},
$$

where $x_{0}=s_{1}+2 \pi-s_{N}$. Find the minimum of the function

$$
\psi\left(x_{0}, \ldots, x_{N}\right) \geq \frac{2}{1+\alpha} \sum_{k=0}^{N-1} x_{k}^{1+\alpha}
$$

under limitation $2 \sum_{k=0}^{N-1} x_{k}=2 \pi$.

It is possible to show that $\psi\left(x_{0}, \ldots, x_{N}\right) \geq 2 \pi^{1+\alpha} /(1+\alpha) N^{\alpha}$ and $\max _{s, j}\left(F \phi_{j}^{*}\right)(s) \geq$ $(1+o(1)) 2 \pi^{\alpha-1} \ln N /(1+\alpha) N^{\alpha}$.

The error estimate of the quadrature rule (1.1) is given by the inequality $R_{N}[\Psi] \leq$ $(2+o(1)) \ln N / \pi^{1-\alpha}(1+\alpha) N^{\alpha}$. This completes the proof.

Proofs of Theorems 3.2, 3.3, and 3.5 are similar to that of Theorem 3.1 but technical realization of these proofs is more difficult. These proofs are given in $[6,8]$.

\subsection{Asymptotically optimal quadrature rules on the class $W^{r}(1)$}

THEOREM 3.7 (see $[6,8])$. Let $\Psi=\tilde{W}^{r}(1)(r=1,2, \ldots$,$) and the integral F \phi$ is evaluated by the quadrature rule (3.2), where $\rho=0,1$. Then $\zeta_{N}[\Psi] \geq 2 K_{r} \pi^{-1} N^{-r} \ln N+$ $o\left(N^{-r}\right)$.

TheOREM 3.8 (see $[6,8]$ ). Let $\Psi=\tilde{W}^{r} L_{p}(1), r=1,2, \ldots, 1 \leq p<\infty$. Let the integral $F \phi$ be calculated with quadrature rules of the type (3.2), where $\rho=r-1, r=1,2, \ldots$, or $\rho=r-2, r=2,4, \ldots$. Then

$$
\zeta_{N}[\Psi]=\frac{(1+o(1)) 2(2 \pi)^{r+1 / q} R_{r q}(1) \ln N}{\pi r !(r q+1)^{q}(2 N)^{r}} .
$$

Consider the quadrature rule

$$
\begin{aligned}
F \phi= & \int_{t_{j-2}}^{t_{j+2}} \tilde{\phi}\left(\sigma,\left[t_{j-2}, t_{j+2}\right]\right) \operatorname{ctg} \frac{\sigma-s}{2} d \sigma \\
& +\sum_{k=0}^{N-1}, \int_{t_{k}}^{t_{k+1}} \tilde{\phi}\left(\sigma,\left[t_{k}, t_{k+1}\right]\right) \operatorname{ctg} \frac{\sigma-s}{2} d \sigma+R_{N},
\end{aligned}
$$

where $t_{k}=2 k \pi / N, k=0,1, \ldots, N ; s \in\left[t_{j}, t_{j+1}\right) ; \tilde{\phi}\left(t,\left[t_{k}, t_{k+1}\right]\right)$ is the local spline that was constructed in Section 1.3, the prime in the summation indicate that $k \neq j-1, j$, $j+1$.

THEOREM 3.9 (see $[6,8])$. Let $\Psi=\tilde{W}^{r}(1), r \geq 3$. Let $s \in\left[t_{j}, t_{j+1}\right)$. Among all quadrature rules of the type (3.2) provided $\rho=r-1$ the quadrature rule (3.18) is asymptotically optimal.

TheOREM 3.10 (see $[6,8]$ ). Let $\Psi=W_{p}^{r}(1), r=1,2, \ldots, 1 \leq p \leq \infty$. Let the integral $K f$ be calculated with quadrature rules of the type (3.4) provided $\rho=0$. Then $\zeta[\Psi] \geq$ $(1+o(1)) 2^{r+1+1 / q} \inf _{c}\left\|B_{r}(\cdot)-c\right\|_{L_{q}[0,1]} \ln N / N^{r}$. 
TheOREM 3.11 (see $[6,8]$ ). Let $\Psi=W_{p}^{r}(1), r=1,2, \ldots, 1 \leq p \leq \infty$. Let the integral $K f$ be calculated with quadrature rules of the type (3.4), where $\rho=r-1, r=1,2, \ldots$, or $\rho=r-2, r=2,4, \ldots$ Then $\zeta[\Psi] \geq 2(1+o(1)) R_{r q}(1)(\ln N) / 2^{r} r !(r q+!)^{1 / q}(N-1+$ $\left.\left(R_{r q}(1)\right)^{1 / r}\right)^{r}$.

Consider a quadrature rule

$$
\begin{aligned}
K f= & \int_{t_{j-2}}^{t_{j+2}} \frac{\tilde{f}\left(\tau,\left[t_{j-2}, t_{j+2}\right]\right)}{\tau-t} d \tau+\sum_{k=0}^{j-3} \frac{\tilde{f}\left(\tau,\left[t_{k}, t_{k+1}\right]\right)}{\tau-t} d \tau \\
& +\sum_{k=j+2}^{N-1} \frac{\tilde{f}\left(\tau,\left[t_{k}, t_{k+1}\right]\right)}{\tau-t} d \tau+R_{N}
\end{aligned}
$$

where $t_{k}=-1+2 k / N, k=0,1, \ldots, N ; t \in\left[t_{j}, t_{j+1}\right), \tilde{f}\left(\tau,\left[t_{k}, t_{k+1}\right]\right)$ is local spline that was constructed in Section 1.3

THEOREM 3.12 (see $[6,8])$. Let $\Psi=W^{r}(1), r=1,2, \ldots$. Among all quadrature rules of the type (3.4), where $\rho=r-1$, the quadrature rule (3.19) is asymptotically optimal and has the error $R_{N}[\Psi]=(1+o(1)) R_{r q}(1)(\ln ((N-j-1)(j-1))) /(r+1) !(2 N)^{r}$.

Consider a quadrature rule

$$
\begin{aligned}
K f= & \frac{1}{v_{j-1}-t}\left(Q_{M, r, \infty}\left(f,\left[v_{j-1}, v_{j}\right]\right)+Q_{M, r, \infty}\left(f,\left[v_{j}, v_{j+1}\right]\right)+Q_{M, r, \infty}\left(f,\left[v_{j+1}, v_{j+2}\right]\right)\right) \\
& +\sum_{k=0}^{L-1} ' \frac{1}{v_{k}-t}\left(f,\left[v_{k}, v_{k+1}\right]\right)+\int_{v_{j-1}}^{v_{j+2}} D_{N, r, \infty}\left(T_{r-1}\left(f,\left[v_{j-1}, v_{j+2}\right], v_{j-1}\right)\right) \frac{d \tau}{\tau-t} \\
& +\sum_{k=0}^{L-1}{ }^{v_{k+1}} \int_{v_{k}}^{v_{N, r, \infty}}\left(T_{r-1}\left(f,\left[v_{k}, v_{k+1}\right], v_{k}\right)\right) \frac{d \tau}{\tau-t}+R_{N},
\end{aligned}
$$

where $M=\left[\ln ^{1 / 2 r} N\right] ; L=[N / M] ; v_{k}=-1+2 k / L, k=0,1, \ldots, L ; t \in\left[v_{j}, v_{j+1}\right)$; the prime in the summation indicate that $k \neq j-1, j, j+1$.

THEOREM 3.13 (see $[6,8])$. Let $\Psi=W^{r}(1), r=1,2, \ldots$. Among all quadrature rules of the type (3.4) provided $\rho=0$ the quadrature rule (3.20), where $\rho=0$ is asymptotically optimal and has the error

$$
R_{N}[\Psi]=(1+o(1)) 2^{r+2} N^{-r} \ln N \inf _{c}\left\|D_{r}(\cdot)-c\right\|_{L_{1}[0,1]} .
$$

The similar statement is correct for singular integrals with Hilbert kernels.

Proof OF TheOrem 3.7. We find the lower bound of the value $\zeta_{N}[\Psi]$. There are two possibilities:

(1) at least one of the values $s_{k+1}-s_{k}$ is not less than $h_{0}=2\left(A^{-1} K_{r}(r+\right.$ 1) $\ln N)^{1 / 2} N^{-1}$

(2) all of these values are separately less than $h_{0}$.

The constant $A$ is defined below.

In the first case assuming without loss of generality that $s_{2}-s_{1} \geq h_{0}$, introduce a function $\phi^{*}(\sigma)=A\left(\left(\sigma-s_{1}\right)\left(s_{2}-\sigma\right)\right)^{r}\left(s_{2}-s_{1}\right)^{-r}$ on the segment $\left[s_{1}, s_{2}\right]$ and $\phi^{*}(\sigma)=0$ 
on other segments. The constant $A$ is selected such that $\left|\left(\phi^{*}\right)^{(r)}\right| \leq 1$. Such constant exists. It is not difficult to see that

$$
\begin{aligned}
\max _{0 \leq s \leq 2 \pi} & \frac{1}{2 \pi} \int_{0}^{2 \pi} \phi^{*}(\sigma) \operatorname{ctg} \frac{\sigma-s}{2} d \sigma \\
\geq & \frac{1}{2 \pi} \int_{0}^{2 \pi} \phi^{*}(\sigma) \operatorname{ctg} \frac{\sigma-s_{1}}{2} d \sigma \\
& \geq \frac{1}{2 \pi} \int_{s_{1}}^{\left(s_{1}+s_{2}\right) / 2} \phi^{*}(\sigma) \operatorname{ctg} \frac{\sigma-s_{1}}{2} d \sigma+\frac{1}{2 \pi} \int_{\left(s_{1}+s_{2}\right) / 2}^{s_{2}} \phi^{*}(\sigma) \operatorname{ctg} \frac{\sigma-s_{1}}{2} d \sigma \\
& \geq \frac{2 K_{r} \ln N}{\pi N^{r}} .
\end{aligned}
$$

As for the second case. Denote by $\phi^{*}(\sigma)$ a function satisfying the following conditions:

(1) $\phi^{*}(\sigma) \in \Psi=\tilde{W}^{r}(1)$;

(2) $\min _{\sigma} \phi^{*}(\sigma)=\phi^{*}\left(s_{k}\right)=0,(k=1,2, \ldots, N)$;

(3) $\int_{0}^{2 \pi} \phi^{*}(\sigma) d \sigma \geq 2 \pi K_{r} / N^{r}$.

The existence of such functions was proved by Motornii in [32]. Divide the segment $[0,2 \pi]$ into $M$ equal portions $\left(M=\left[2 \pi / h_{0}\right]\right)$ by the points $v_{k}=2 k \pi / M, k=0,1, \ldots, M$. In each segment it exists at least a single node of the quadrature rule (3.2). Denote this point by $s_{k}^{*}$. Take an arbitrary number $j(0 \leq j \leq M)$ and select the segments $\left[s_{j-1}^{*}, s_{j+1}^{*}\right],\left[\bar{s}_{j-1}^{*}, \bar{s}_{j+1}^{*}\right]$, moreover $s_{j}^{*}+\pi \subset\left[\bar{s}_{j-1}^{*}, \bar{s}_{j+1}^{*}\right]$. Denote by $\psi_{j}^{*}(\sigma)$ a function satisfying the following conditions:

(1) $\psi_{j}^{*}(\sigma) \in \Psi=\tilde{W}^{r}$;

(2) on the segment $\left[s_{j+1}^{*}, \bar{s}_{j-1}^{*}\right] \psi_{j}^{*}(\sigma)=\phi^{*}(\sigma)$;

(3) on the segment $\left[\bar{s}_{j+1}^{*}, s_{j-1}^{*}\right] \psi_{j}^{*}(\sigma)=-\phi^{*}(\sigma)$.

For $r=1,2$ the function $\psi_{j}^{*}(\sigma)$ on the segments $\left[s_{j-1}^{*}, s_{j+1}^{*}\right],\left[\bar{s}_{j-1}^{*}, \bar{s}_{j+1}^{*}\right]$ can be assumed to be equal to zero. It follows from the fact that at the points $s_{k}^{*}$ ( $k=$ $0,1, \ldots, N)$ the function $\phi^{*}(\sigma)$ and their first derivative are equal to zero. In the explicit form the function $\psi_{j}^{*}(\sigma)$ can be constructed for $r=3$.

However it was not succeeded in making for $r \geq 4$. Therefore to prove the existence of the required function is naturally to take advantage of the "cut-off function method" applied by Sobolev in [43, page 697] in investigating quadrature rule with boundary layer. Select the segments $\left[s_{j-N_{1}}^{*}, s_{j+N_{1}}^{*}\right],\left[\bar{s}_{j-N_{1}}^{*}, \bar{s}_{j+N_{1}}^{*}\right]$, moreover a value $N_{1}$ will be defined below.

Introduce a function

$$
l_{j}(s)= \begin{cases}1, & s \in\left[s_{j+N_{1}}^{*}, \bar{s}_{j-N_{1}}^{*}\right], \\ -1, & s \in\left[\bar{s}_{j+N_{1}}^{*}, s_{j-N_{1}}^{*}\right], \\ 0, & s \in[0,2 \pi] \backslash\left[s_{j+N_{1}}^{*}, \bar{s}_{j-N_{1}}^{*}\right] \cup\left[\bar{s}_{j+N_{1}}^{*}, s_{j-N_{1}}^{*}\right] .\end{cases}
$$

Now construct a "cutting off" multiplier $\delta_{j}(s)$ that is equal to unit on the segment $\left[s_{j+3 N_{1}}^{*}, s_{j-3 N_{1}}^{*}\right]$; that is equal to minus unit on the segment $\left[\bar{s}_{j+3 N_{1}}^{*}, s_{j-3 N_{1}}^{*}\right]$ and has continuous derivatives of all orders. In addition we require that $\delta_{j}(s) \phi(s) \in W^{r}(1+$ $o(1))$. In the capacities of function $\delta_{j}(s)$ we will take a mean function (with infinitely 
differentiable kernel of average) with average radius $h=2 N_{1} \pi / M$ for function $l_{j}(s)$. It is not difficult to see that at the points vicinity $s_{j}^{*}, \bar{s}_{j}^{*}$ the function $\delta_{j}(s)$ is equal to zero, on the segments $\left[s_{j+3 N_{1}}^{*}, \bar{s}_{j-3 N_{1}}^{*}\right]$, and $\left[\bar{s}_{j+3 N_{1}}^{*}, s_{j-3 N_{1}}^{*}\right]$ it is, respectively, equal to unit and minus unit and has derivatives of all orders.

Remember that by definition a mean function is given by

$$
\delta_{j}^{h}(s)=\kappa h \int_{s-\pi}^{s+\pi} \omega\left(\frac{s-\sigma}{h}\right) l_{j}(\sigma) d \sigma
$$

where $\omega((s-\sigma) / h)$ is the average kernel satisfying the norm requirements (Sobolev [43, page 104]). It follows from definition of the function $\delta_{j}^{h}(s)$ that

$$
\frac{d^{r} \delta_{j}^{h}(s)}{d s^{r}}=\kappa h^{1-r} \int_{s-\pi}^{s+\pi} \omega^{(r)}\left(\frac{s-\sigma}{h}\right) l_{j}(\sigma) d \sigma=0\left(h^{-r}\right) .
$$

Find norm $\delta_{j}^{h}(s) \phi^{*}(s)$. First of all notice that since the function $\phi^{*}(s)$ has derivative of order $r$ not exceeding a unit module and at $N$ points distant from each other by not more than $h_{0}$, is equal to zero, then its derivatives of order $r-1$ vanish not less than at $N-r+1$ points distant each other by not more than $2^{r-1} h_{0}$. Since $\left|\phi^{*(r)}(s)\right| \leq$ 1 then $\left|\phi^{*(r-1)}(s)\right| \leq 2^{r-1} h_{0},\left|\phi^{*(r-2)}(s)\right| \leq 2^{2 r-3} h_{0}^{2}, \ldots,\left|\phi^{*(s)}\right| \leq 2^{r^{2} / 2} h_{0}^{r}$. Therefore $\left|\left(\delta_{j}(s) \phi^{*(r)}(s)\right)\right|=\left|\delta_{j}^{(r)}(s) \phi^{*}(s)+C_{r}^{1} \delta_{j}^{(r-1)}(s) \phi^{*(1)}(s)+\cdots+\delta_{j}(s) \phi^{*(r)}(s)\right|$.

On the segments $\left[s_{j+N_{1}}^{*}, \bar{s}_{j-N_{1}}^{*}\right],\left[\bar{s}_{j+N_{1}}^{*}, s_{j-N_{1}}^{*}\right]$, the formula $\left|\left(\delta_{j}(s) \phi^{*(r)}(s)\right)\right|=$ $\left|\delta_{j}(s) \phi^{*(r)}(s)\right| \leq 1$ is valid and for other values $\left|\left(\delta_{j}(s) \phi(s)\right)^{(r)}\right|=o\left(h^{-r} h_{0}^{r}+h^{1-r} h_{0}^{r-1}\right.$ $\left.+\cdots+h^{-1} h_{0}\right)+1$. Now assume $h=h_{0} \ln N$. Then $\left|\left(\delta_{j}(s) \phi^{*}(s)\right)^{(r)}\right|=1+o(1)$ for all $j$. Therefore $N_{1}=[\ln N]+1$.

Fix a positive arbitrarily small $\epsilon(0<\epsilon<1)$ and let $\beta=1-\epsilon$. The function $\psi_{j}(s)=$ $\beta \delta_{j}(s) \phi^{*}(s)$ satisfies all formulated requirements. Therefore having repeated the arguments made in proving Theorem 3.1, we have

$$
\sup _{s, j} F \psi_{j}(s) \geq \frac{2(1+o(1))(1-\epsilon) K_{r} \ln N}{\pi N^{r}} .
$$

It follows from arbitrariness of $\epsilon$ that the final estimation holds

$$
\sup _{\phi \in \tilde{W}^{r}(1)} F \phi \geq \frac{(2+o(1)) K_{r} \ln N}{\pi N^{r}} .
$$

This completes the proof.

3.4. Optimal with respect to order quadrature rules. In computing singular integrals by asymptotically optimal quadrature rules constructed in the Sections 3.2, 3.3 it is necessary to evaluate the coefficients $p_{k}(s)$ for every value of $s$.

Construct less exact but more easy realizable algorithms. Divide the segment $[0,2 \pi]$ into $N$ equal parts by the points $t_{k}=2 k \pi / N, k=0,1, \ldots, N$. Let $t_{k}^{\prime}=(2 k+1) \pi / N$, $k=0,1, \ldots, N$. Let the point $s$ lies in the segment $\left[t_{j}, t_{j+1}\right]$. The integral $F \phi$ we shall calculate by the formula

$$
\frac{1}{2 \pi} \int_{0}^{2 \pi} \phi(\sigma) \operatorname{ctg} \frac{\sigma-s}{2} d \sigma=\frac{1}{\pi} \sum_{k=1}^{N} \phi\left(t_{k}^{\prime}\right) \ln \frac{\sin \pi((2 k-2 j-1) / 2 N)}{\sin (\pi(2 k-2 j-3) / 2 N)}+R_{N} .
$$


THEOREM 3.14 (see $[6,8])$. Let $\Psi=H_{\alpha}(1)(0<\alpha \leq 1)$. Among all quadrature rules of the type (3.2) with $\rho=0$ the quadrature rule (3.28) is asymptotically optimal for $0<\alpha<1$ and optimal with respect to order for $\alpha=1$. Its error is equal to $R_{N}[\Psi] \leq$ $2 \ln N / \pi^{1-\alpha}(1+\alpha) N^{\alpha}+O\left(N^{-\alpha}\right)$.

In computing singular integrals, the quadrature rules is based on the change of the integrand function by some approximating assembly. Changing of the integrand function by Lagrange interpolated polynomial constructed on $2 N+1$ equidistant point $s_{k}=2 k \pi /(2 N+1), k=0,1, \ldots, 2 N$ is used particularly often in (see [23, 27]). The interpolated polynomial is given in $[34,35]$ by the formula

$$
P_{N}[\phi]=\sum_{k=0}^{2 N} \phi\left(s_{k}\right) \psi_{k}(s), \psi_{k}(s)=\frac{1}{2 N+1} \frac{\sin (2 N+1 / 2)\left(s-s_{k}\right)}{\sin \left(s-s_{k} / 2\right)} .
$$

The quadrature rule has the form

$$
F \phi=\frac{1}{2 \pi} \int_{0}^{2 \pi} P_{N}[\phi(\sigma)] \operatorname{ctg} \frac{\sigma-s}{2} d \sigma+R_{N}(s)
$$

This quadrature rule can be represented as the finite summation

$$
F \phi=-\frac{2}{2 N+1} \sum_{k=0}^{2 N} \phi\left(s_{k}\right) \frac{\sin (N+1)\left(s-s_{k} / 2\right) \sin N\left(s-s_{k} / 2\right)}{\sin \left(s-s_{k} / 2\right)} .
$$

Assume that function $\phi(t)$ is representable in convolution form

$$
\phi(x)=\int_{-\pi}^{\pi} G(x-t) h(t) d t
$$

where $h(t)$ is continuous $2 \pi$-periodic function such that $\max _{-\pi \leq t \leq \pi}|h(t)| \leq 1$, $\operatorname{essmax}_{-\pi \leq t \leq \pi}|\tilde{h}(t)| \leq K ; \tilde{h}(t)$ is the function conjugate $h(t) ; G(t)$ has property $A_{N+1}$.

DEFINITION 3.15. The function $G(x)$ has the property $A_{N+1}$ provided

(a) $G(x)$ is even and for any $a_{k}, k=0,1, \ldots, N$, the function $G(x)-\sum_{0}^{N} a_{k} \cos k x$ cannot have more than $N+1$ nodes in the interval $(0, \pi)$;

(b) $G(x)$ is odd and for any $a_{k}, k=0,1, \ldots, N$, the function $G(x)-\sum_{1}^{N} a_{k} \sin k x$ cannot have more than $N$ nodes in the interval $(0, \pi)$.

THEOREM 3.16 (see $[6,8]$ ). Let $\phi(t)$ belong to the class of functions represented in convolution form of (3.32) and $\max _{-\pi \leq t \leq \pi}|h(t)| \leq 1$, $\operatorname{essmax}_{-\pi \leq t \leq \pi}|\tilde{h}(t)| \leq K$. Then the quadrature rule (3.30) error is estimated by the inequality $\left|R_{N}\right| \leq K E_{N, 1}(G)+$ $\pi^{-1} E_{N}(\phi)(\ln N+C)$, where $E_{N, P}(G)$ is the best approximation of the function $G(t)$ by trigonometric polynomials of degree $N$ in the metric $L_{p}, E_{N}=E_{N, \infty}, C=0,577215$.

THEOREM 3.17 (see $[6,8])$. Let $\phi \in \tilde{W}_{p}^{r}(1), 1 \leq p \leq 2$. Then the error of quadrature rule (3.30) is estimated by the inequality $\left|R_{N}\right| \leq E_{N, p^{1}}\left(D_{r}\right)\left(2 p^{\prime}+\pi^{-r} \ln N\right)$, where $D_{r}(x)$ are Bernoulli polynomials, $1 / p+1 / p^{\prime}=1$. 
Proofs of these theorems are given in $[6,8]$. At the end of the section we introduce optimal with respect to order algorithm of evaluation of the singular integral $K f$, which is based on Gauss quadrature rule. This algorithm is very simple in application. Convergence of this algorithm is given in [12].

We rewrite the integral $K f$ as

$$
\begin{aligned}
K f= & \int_{-1}^{1} \frac{f(\tau) d \tau}{\tau-t} \\
= & \int_{-1}^{1} \frac{g(\tau) d \tau}{\tau-t}-\left(f(1)+\frac{1}{2} f(-1)\right) \\
& \times \ln \left|\frac{1-t}{1+t}\right|-\frac{f(1)-f(-1)}{2}\left(2+t \ln \left|\frac{1-t}{1+t}\right|\right)
\end{aligned}
$$

and we will construct numerical algorithm for evaluation of the integral

$$
K g=\int_{-1}^{1} \frac{g(\tau) d \tau}{\tau-t}
$$

where $g(\tau)=f(\tau)-(\tau+1) / 2[f(1)-f(-1)]-f(-1)$.

We now construct continuous local spline that approximate the function $K f(t)$ with accuracy $A n^{-r} \ln n$, where $n$ is the number of functionals $f\left(t_{k}\right)$, used in the construction of the algorithm. For this purpose, we divide the segment $[-1,1]$ into $2 n$ parts by the points $t_{k}=-1+(k / n)^{v}$ and $\tau_{k}=1-(k / n)^{v}$, where $k=0,1, \ldots, n$ and $v=r$. We construct interpolating polynomial whose interpolation points contain the endpoints of the interpolation segment.

The polynomial $P_{r}(f,[a, b])$ that interpolated the function $f(t)$ on the segment $[a, b]$ is constructed as follows. Denote by $\zeta_{k}, k=1,2, \ldots, r$ roots of the Legendre polynomial of degree $r$. We map the segment $\left[\zeta_{1}, \zeta_{r}\right] \in[-1,1]$ onto $[a, b]$ so that the points $\zeta_{1}$ and $\zeta_{r}$ map to $a$ and $b$, respectively. Images of the points $\zeta_{i}$ under this mapping are denoted by $\zeta_{i}^{\prime}, i=1,2, \ldots, r$. Using the points of $\zeta_{i}^{\prime}, i=1,2, \ldots, r$, we construct the interpolation polynomial $P_{r}(f,[a, b])$ of degree $r-1$.

We divide segments $\left[-1, t_{1}\right]$ and $\left[\tau_{1}, 1\right]$ onto $M=[\ln N]$ parts by the points $t_{0, l}=-1+$ $l t_{1} / M$ and $\tau_{0, l}=1-l \tau_{1} / M, l=0,1, \ldots, M$, respectively. The function $G(t)=(K g)(t)$ is approximated by interpolation polynomials $P_{r}\left(\tilde{G}(t), \Delta_{0, l}\right)$ and $P_{r}\left(\tilde{G}(t), \Delta_{0, l}^{*}\right)$ on the segments $\Delta_{0, l}=\left[t_{0, l}, t_{0, l+1}\right]$ and $\Delta_{0, l}^{*}=\left[\tau_{0, l+1}, \tau_{0, l}\right], l=0,1, \ldots, M-1$, respectively.

The function $G(t)=(K g)(t)$ is approximated by interpolation polynomials $P_{r}\left(\tilde{G}(t), \Delta_{k}\right)$ and $P_{r}\left(\tilde{G}(t), \Delta_{k}^{*}\right)$ on the segments $\Delta_{k}=\left[t_{k}, t_{k+1}\right]$ and $\Delta_{k}^{*}=\left[\tau_{k+1}, \tau_{k}\right]$, $l=0,1, \ldots, n-1$, respectively.

The values $\tilde{G}\left(\zeta_{k, l}\right)$ and $\tilde{G}\left(\zeta_{k, l}^{*}\right)$, where $\zeta_{k, l}$ and $\zeta_{k, l}^{*}$ are nodes of the interpolation polynomials $P_{r}\left(\tilde{G}(t), \Delta_{k}\right)$ and $P_{r}\left(\tilde{G}(t), \Delta_{k}^{*}\right)$, are found from quadrature rules

$$
\tilde{G}\left(\zeta_{k, j}\right)=\sum_{l=0}^{N-1} \int_{w_{l}}^{w_{l+1}} P_{r}\left(\frac{g(\tau)-g\left(\zeta_{k, j}\right)}{\tau-\zeta_{k, j}},\left[w_{l}, w_{l+1}\right]\right) d \tau+g\left(\zeta_{k, j}\right) \ln \left|\frac{1-\zeta_{k, j}}{1+\zeta_{k, j}}\right|,
$$

where $w_{l}=-1+2 l / n, l=0,1, \ldots, n$.

The error of this algorithm is equal to $A \ln N / N^{r}$. 
4. Optimal quadrature rules for calculating polysingular and many-dimensional singular integrals

4.1. Optimal algorithms for calculating integrals with fixed singularity. Consider quadrature rules of the following type:

$$
S f=\int_{-1}^{1} \int_{-1}^{1} \frac{f\left(t_{1}, t_{2}\right)}{t_{1} t_{2}} d t_{1} d t_{2}=\sum_{k, l=-N, k, l \neq 0}^{N} p_{k l} f\left(t_{k}, t_{l}\right)+R_{N N}\left(f ; p_{k l} ; t_{k}, t_{l}\right),
$$

where $t_{k}=-t_{-k}, p_{k, l}=p_{-k,-l}=-p_{-k, l}=-p_{k,-l}, k, l=-N, \ldots,-1,1, \ldots, N$.

THEOREM 4.1 (see [6]). Let $\Psi=W^{1,1}(1)$. Among all quadrature rules of the type (4.1) with $t_{-N}=-1, t_{N}=1$ the formula

$S f=\sum_{j=1}^{N} \sum_{k=1}^{N} 4 \ln \frac{j+1}{j} \ln \frac{k+1}{k}\left[f\left(v_{j}, v_{k}\right)-f\left(-v_{j}, v_{k}\right)-f\left(v_{j},-v_{k}\right)+f\left(-v_{j},-v_{k}\right)\right]+R_{N N}$,

where $v_{k}=k(k+1) / N(N+1), k=1,2, \ldots, N$ is optimal and has the error

$$
R_{N N}[\Psi] \leq \frac{8}{N+1} \text {. }
$$

REMARK 4.2 (see [42]). The exact value of $R_{N N}[\Psi]$ is equal to $R_{N N}[\Psi]=8 \ln (1+$ $1 / N)+4 \ln ^{2}(1+1 / N)$.

THEOREM 4.3 (see [6]). Let $\Psi=W^{1,1}(1)$. Among all quadrature rules of the type (4.1) the formula

$$
S f=\sum_{j=1}^{N} \sum_{k=1}^{N} 4 \ln \frac{j+1}{j} \ln \frac{k+1}{k}\left[f\left(v_{j}, v_{k}\right)-f\left(-v_{j}, v_{k}\right)-f\left(v_{j},-v_{k}\right)+f\left(-v_{j},-v_{k}\right)\right]+R_{N N},
$$

where $v_{k}=k(k+1) /(N+1)^{2}, k=1,2, \ldots, N$, is optimal and has the error equal to $R_{N N}[\Psi] \leq 8 / N+1+4 \ln N /(N+1)^{2}$.

REMARK 4.4 (see [42]). The exact value of $R_{N N}[\Psi]$ is equal to $R_{N N}[\Psi]=8 /(N+1)+$ $4 /(N+1)^{2}$.

Consider singular integrals

$$
I f=\int_{-1}^{1} \cdots \int_{-1}^{1} \frac{f\left(t_{1}, \ldots, t_{l}\right)}{t_{1} \cdots t_{l}} d t_{1} \cdots d t_{l} .
$$

We will calculate the singular integrals $I f$ with quadrature rules

$$
\begin{aligned}
& I f=\sum_{k_{1}=1}^{N} \cdots \sum_{k_{l}=1}^{N} p_{k_{1}, \ldots, k_{l}} f\left(t_{k_{1}}, \ldots, t_{k_{l}}\right)+R_{N}, \\
& I f=\sum_{k_{1}=1}^{N} \cdots \sum_{k_{l}=1}^{N} \sum_{i_{1}=0}^{\rho_{1}} \cdots \sum_{i_{l}=0}^{\rho_{l}} p_{k_{1}, \ldots, k_{l}, i_{1}, \ldots, i_{l}} \frac{\partial^{i_{1}+\cdots+i_{l}} f\left(t_{k_{1}}, \ldots, t_{k_{l}}\right)}{\partial t_{1}^{i_{1}} \cdots \partial t_{l}^{i_{l}}}+R_{N}, \\
& I f=\sum_{k=1}^{N} p_{k} f\left(M_{k}\right)+R_{N}, \quad M_{k} \in[-1,1]^{l} .
\end{aligned}
$$


THEOREM 4.5 (see [6]). Let $\Psi=H_{\alpha, \ldots, \alpha}(1)$. Let the integral If be calculated with quadrature rules of the type (4.6). Then

$$
\zeta_{N}[\Psi] \geq \frac{(1+o(1)) 2^{l-2 \alpha}(1+\alpha)^{1-l} \ln ^{l-1} N}{\alpha N^{\alpha}} .
$$

THEOREM 4.6 (see [6]). Let $\Psi=C_{l}^{r}, r=1,2, \ldots$ Let the integral $f f$ be calculated with quadrature rule of the type (4.6). Then $\zeta_{N}[\Psi] \geq A N^{-r} \ln ^{l-1} N$.

THEOREM 4.7 (see [6]). Let $\Psi=C_{l}^{r}, r=1,2, \ldots$ Let the integral If be calculated with quadrature rule of the type (4.7). Then $\zeta_{N}[\Psi] \geq A(r N)^{-r} \ln ^{l-1} N$.

TheOrem 4.8 (see [6]). Let $\Psi=Z_{i}^{\alpha}$ or $\Psi=H_{i}^{\alpha}, i=1,2,3$. Let the integral If be calculated with quadrature rule of the type (4.8). Assume that the integral If is twodimensional integral $(l=2)$ when the function $f$ belongs to the classes $H_{i}^{\alpha}, i=2,3$ or $Z_{i}^{\alpha}, i=2,3$. Then $\zeta_{N}\left(H_{i}^{\alpha}\right)=2 \zeta_{N}\left(Z_{i}^{\alpha}\right)=D_{i}(2(l+\alpha) / \alpha)^{l+\alpha} N^{-\alpha / l}+o\left(N^{-\alpha / l}\right)$, where

$$
D_{1}=\frac{l}{(l+\alpha) 2^{\alpha}}, \quad D_{2}=\frac{2^{1-\alpha / 2}}{2+\alpha}, \quad D_{3}=\frac{12}{2+\alpha}\left(\frac{1}{2 \sqrt{3}}\right)^{(\alpha+2) / 2} \int_{0}^{\pi / 6} \frac{d t}{\cos ^{2+\alpha} t} .
$$

We construct some optimal with respect to order quadrature rules for calculation of the integral $I f$.

Theorem 4.9 (see [6]). Let $\Psi=H_{\alpha, \alpha}(1), 0<\alpha \leq 1$. Let $l=2$. Among all possible quadrature rules of the type (4.6) the formula

$$
I f=\sum_{k=-N, k \neq-1,0}^{N-1} \sum_{i=-N, i \neq-1,0}^{N-1} f\left(v_{k}^{\prime}, v_{i}^{\prime}\right) \iint_{\Delta_{k i}} \frac{d t_{1} d t_{2}}{t_{1} t_{2}}+R_{N},
$$

where $v_{ \pm k}= \pm(k / N)^{(1+\alpha) / \alpha}, k=0,1, \ldots, N ; v_{k}^{\prime}=\left(v_{k+1}+v_{k}\right) / 2, \Delta_{i j}=\left[v_{i}, v_{i+1} ; v_{j}, v_{j+1}\right]$, $i, j=-N, \ldots,-1,0,1, \ldots, N-1$, is optimal with respect to order and has the error

$$
\left|R_{N}\right| \leq(8+o(1))\left(\frac{1+\alpha}{\alpha}\right)^{2+\alpha} \frac{\ln N}{2^{\alpha} N^{\alpha}}
$$

Let $D=[-1,1]^{2}$. Let $f\left(t_{1}, t_{2}\right)\left(\left(t_{1}, t_{2}\right) \in D\right)$ be a function from the class $C_{2}^{r}, r=$ $1,2, \ldots$ We construct a local spline for approximation of the function $f\left(t_{1}, t_{2}\right)$. Let $\Delta_{i j}=\left[v_{i}, v_{i+1} ; v_{j}, v_{j+1}\right], i, j=-N, \ldots, N-1$, where $v_{ \pm k}= \pm(k / N)^{(r+1) / r}, k=0,1, \ldots, N$. In each domain $\Delta_{i j}$ we approximate the function $f\left(t_{1}, t_{2}\right)$ with Taylor series $T_{r-1} \times$ $\left(f, \Delta_{i j},\left(v_{i}^{\prime}, v_{j}^{\prime}\right)\right)$, where $v_{i}^{\prime}=\left(v_{i}+v_{i+1}\right) / 2$.

Let $f_{N}\left(t_{1}, t_{2}\right)\left(t_{1}, t_{2}\right) \in D$ be local spline which consists of polynomials $T_{r-1}\left(f, \Delta_{i, j}\right)$, $i, j=-N, \ldots, N-1$.

THEOREM 4.10 (see [6]). Let $\Psi=C_{2}^{r}, r=1,2, \ldots$. Among all possible quadrature rules of the type (4.7) the formula If $=I f_{N}+R_{N}$ is optimal with respect to order and has the error $\left|R_{N}\right|=A N^{-r} \ln N$.

Proofs of Theorems 4.1, 4.3, 4.5, 4.6, 4.7, 4.8, 4.9, and 4.10 are given in [6, 8]. 
4.2. Approximate methods of the calculation of the polysingular integrals with Cauchy and Hilbert kernels. In this item we investigate optimal methods of the calculation of the polysingular integrals with Hilbert kernel as

$$
H f=\frac{1}{\left(2 \pi^{2}\right)^{l}} \int_{0}^{2 \pi} \cdots \int_{0}^{2 \pi} f\left(\sigma_{1}, \ldots, \sigma_{l}\right) \operatorname{ctg} \frac{\sigma_{1}-s_{1}}{2} \cdots \operatorname{ctg} \frac{\sigma_{l}-s_{l}}{2} d \sigma_{1} \cdots d \sigma_{l},
$$

the polysingular integrals with Cauchy kernel as

$$
G f=\int_{0}^{1} \cdots \int_{0}^{1} \frac{f\left(\tau_{1}, \ldots, \tau_{l}\right) d \tau_{1} \cdots d \tau_{l}}{\left(\tau_{1}-t_{1}\right) \cdots\left(\tau_{l}-t_{l}\right)}
$$

and the many-dimensional singular integrals as

$$
K f=\int_{D} \frac{\phi(\theta) f\left(\tau_{1}, \ldots, \tau_{l}\right) d \tau_{1} \cdots d \tau_{l}}{\left(\left(\tau_{1}-t_{1}\right)^{2}+\cdots+\left(\tau_{l}-t_{l}\right)^{2}\right)^{l / 2}}, \quad D=[0,1]^{l}, l=2,3, \ldots
$$

For calculating integrals as (4.13) we will use quadrature rules of the following types:

$$
\begin{array}{r}
H f=\sum_{k_{1}=1}^{n_{1}} \cdots \sum_{k_{l}=1}^{n_{l}} \sum_{i_{1}=0}^{\rho_{1}} \cdots \sum_{i_{l}=0}^{\rho_{l}} p_{k_{1} \cdots k_{l}, i_{1}, \ldots, i_{l}}\left(s_{1}, \ldots, s_{l}\right) f^{i_{1}, \ldots, i_{l}}\left(x_{k_{1}}, \ldots, x_{k_{l}}\right) \\
+R_{n}\left(s_{1}, \ldots, s_{l} ; x_{k_{1}}, \ldots, x_{k_{l}} ; p_{\left.k_{1}, \ldots, k_{l}, i_{1}, \ldots, i_{l}, f\right)},\right.
\end{array}
$$

where $0 \leq x_{1}<x_{2}<\cdots<x_{m} \leq 2 \pi$, and

$$
H f=\sum_{k=1}^{N} p_{k}\left(s_{1}, \ldots, s_{l}\right) f\left(M_{k}\right)+R_{N}\left(s_{1}, \ldots, s_{l} ; p_{k}, M_{k}, f\right),
$$

where $M_{k} \in D=[0,2 \pi]^{l}, k=1,2, \ldots, N$.

For calculating the integrals (4.14) and (4.15) we will use quadrature rules of the following types

$$
\begin{array}{r}
G f=\sum_{k_{1}=1}^{n} \ldots \sum_{k_{l}=1}^{n} \sum_{i_{1}=0}^{\rho_{1}} \ldots \sum_{i_{l}=0}^{\rho_{l}} p_{k_{1} \cdots k_{l}, i_{1} \cdots i_{l}}\left(t_{1}, \ldots, t_{l}\right) f^{\left(i_{1}, \ldots, i_{l}\right)}\left(t_{k_{1}}, \ldots, t_{k_{l}}\right) \\
+R_{n}\left(t_{1}, \ldots, t_{l} ; p_{k_{1} \cdots k_{l} i_{1} \cdots i_{l}} ; t_{k_{1}}, \ldots, t_{k_{l}} ; f\right) ; \\
G f=\sum_{k=1}^{N} p_{k}\left(t_{1}, \ldots, t_{l}\right) f\left(M_{k}\right)+R_{N}\left(t_{1}, \ldots, t_{l} ; p_{k} ; M_{k} ; f\right), \\
K f=\sum_{k_{1}=1}^{n} \ldots \sum_{k_{l}=1}^{n} \sum_{i_{1}=0}^{\rho_{1}} \cdots \sum_{i_{l}=0}^{\rho_{l}} p_{k_{1} \cdots k_{l} i_{1} \cdots i_{l}}\left(t_{1}, \ldots, t_{l}\right) f^{\left(i_{1}, \ldots, i_{l}\right)}\left(t_{k_{1}}, \ldots, t_{k_{l}}\right) \\
\quad+R_{n}\left(t_{1}, \ldots, t_{l} ; p_{k_{1} \cdots k_{l} i_{1} \cdots i_{l}} ; t_{k_{1}}, \ldots, t_{k_{l}} ; f\right) ; \\
K f=\sum_{k=1}^{N} p_{k}\left(t_{1}, \ldots, t_{l}\right) f\left(M_{k}\right)+R_{N}\left(t_{1}, \ldots, t_{l} ; p_{k} ; M_{k} ; f\right) .
\end{array}
$$


4.2.1. Asymptotically optimal quadrature rules on Hölder classes of functions. Consider the polysingular integrals of the type (4.13), where $l=2$. We restricted ourselves to two-dimensional integrals only for simplicity.

THEOREM 4.11 (see $[6,8]$ ). Let $\Psi=H_{\alpha, \alpha}(D)$ or $\Psi=H_{3}^{\alpha}(D)$. Let the integral (4.13) be calculated with quadrature rules of the type (4.16), where $n=n_{1}=n_{2}, \rho_{1}=\rho_{2}=0$. Then

$$
\begin{gathered}
\zeta_{N}\left[H_{\alpha, \alpha}(D)\right] \geq \frac{(1+o(1)) 8 \pi^{\alpha-2}}{(1+\alpha) n^{\alpha}} \ln ^{2} n \\
\zeta_{N}[\Psi] \geq(4+o(1)) \frac{n^{2} \ln ^{2} n}{\pi^{4}} \int_{0}^{\pi / n} \int_{0}^{\pi / n}\left(t^{2}+\tau^{2}\right)^{\alpha / 2} d t d \tau .
\end{gathered}
$$

THEOREM 4.12 (see $[6,8]$ ). The quadrature rule

$$
H f=\frac{1}{4 \pi^{2}} \sum_{k=0}^{n-1} \sum_{l=0}^{m-1} f\left(t_{k}^{\prime}, \tau_{l}^{\prime}\right) \iint_{\Delta_{k l}^{*}} \operatorname{ctg} \frac{\sigma_{1}-s_{1}}{2} \operatorname{ctg} \frac{\sigma_{2}-s_{2}}{2} d \sigma_{1} d \sigma_{2}+R_{n m},
$$

where $\Delta_{k l}=\left[t_{k}, t_{k+1} ; \tau_{l}, \tau_{l+1}\right] ; t_{k}=2 k \pi / n, t_{k}^{\prime}=(2 k+1) \pi / n, k=0,1, \ldots, n ; \tau_{l}=$ $2 l \pi / m, \tau_{l}^{\prime}=(2 l+1) \pi / m, l=0,1, \ldots, m ; \Delta_{0}=\left[s_{1}-4 \pi / n, s_{1}+4 \pi / n ; 0,2 \pi\right] \cup$ $\left[0,2 \pi ; s_{2}-4 \pi / m, s_{2}+4 \pi / m\right], \Delta_{0}^{*}=D \backslash \Delta_{0} ; \Delta_{k l}^{*}=\Delta_{k l} \cap \Delta_{0}^{*}$, has the error

$$
\begin{aligned}
R_{m n}\left[H_{\alpha_{1} \alpha_{2}}(1)\right] \leq & \frac{4}{\pi^{2}}\left(\frac{\pi^{\alpha_{1}}}{\left(1+\alpha_{1}\right) n^{\alpha_{1}}}+\frac{\pi^{\alpha_{2}}}{\left(1+\alpha_{2}\right) m^{\alpha_{2}}}\right) \\
& \times(4+(\ln n+o(\ln n))(\ln m+o(\ln m))), \\
R_{n m}\left[H_{3}^{\alpha}(D)\right] \leq & \frac{4}{\pi^{4}} n m((\ln n+o(\ln n))(\ln m+o(\ln m))+4) \\
& \times \int_{0}^{\pi / n} \int_{0}^{\pi / m}\left(t^{2}+\tau^{2}\right)^{\alpha / 2} d t d \tau .
\end{aligned}
$$

In the case when $\alpha_{1}=\alpha_{2}$ and $n=m$ the quadrature rule (4.23) is asymptotically optimal on classes of functions $H_{\alpha \alpha}(1), H_{3}^{\alpha}(D)$ and has the error

$$
\begin{aligned}
& R_{n n}\left[H_{\alpha \alpha}(1)\right]=(1+o(1)) \frac{8 \pi^{\alpha-2} \ln ^{2} n}{(1+\alpha) n^{\alpha}} \\
& R_{n n}\left[H_{3}^{\alpha}(D)\right]=(4+o(1)) \frac{n^{2} \ln ^{2} n}{\pi^{4}} \int_{0}^{\pi / n} \int_{0}^{\pi / n}\left(t^{2}+\tau^{2}\right)^{\alpha / 2} d t d \tau .
\end{aligned}
$$

THEOREM 4.13 (see $[6,8]$ ). Let $\Psi=H_{\omega \omega}(D)$. In the case $n=m$ the quadrature rule (4.23) is asymptotically optimal and has the error

$$
\begin{aligned}
R_{n n}\left[H_{\omega \omega}(D)\right] & =(8+o(1)) \frac{n \ln ^{2} n}{\pi^{3}} \int_{0}^{\pi / n} \omega(t) d t \\
R_{n n}\left[H_{3}^{\omega}(D)\right] & =(4+o(1)) \frac{n^{2} \ln ^{2} n}{\pi^{4}} \int_{0}^{\pi / n} \int_{0}^{\pi / n} \omega\left(\sqrt{t^{2}+\tau^{2}}\right) d t d \tau
\end{aligned}
$$


TheOREM 4.14 (see $[6,8]$ ). Let $\Psi=H_{i}^{\alpha}$ or $\Psi=Z_{i}^{\alpha}, i=1,2,3$. Let the integral (4.13) be calculated with quadrature rules of type (4.17). Assume that $l=2$ if $i=2,3$. Then $\zeta_{N}\left[H_{i}^{\alpha}\right]=2 \zeta_{N}\left[Z_{i}^{\alpha}\right]=(1+o(1))\left(2^{l+\alpha} D_{i} \pi^{\alpha-l}\right) /\left(l^{l} N^{\alpha / l}\right) \ln ^{l} N$, where constants $D_{1}, D_{2}$, $D_{3}$ was defined in Theorem 4.8 .

THEOREM 4.15 (see $[6,8])$. Let $\Psi=H_{1}^{\alpha}(0<\alpha \leq 1)$. The quadrature rule $H f=\sum_{k_{1}=0}^{n-1} \cdots \sum_{k_{l}=0}^{n-1} f\left(t_{k_{1}}^{\prime}, \ldots, t_{k_{l}}^{\prime}\right) \int \cdots \int_{\Delta_{k_{1}, \ldots, k_{l}}^{*}} \operatorname{ctg} \frac{\sigma_{1}-s_{1}}{2} \cdots \operatorname{ctg} \frac{\sigma_{l}-s_{l}}{2} d \sigma_{1} \cdots d \sigma_{l}+R_{n \cdots n}$,

where $n=\left[N^{1 / l}\right] ; t_{k}=2 k \pi / n, t_{k}^{\prime}=(2 k+1) \pi / n, k=0,1, \ldots, n ; \Delta_{0}=\left[s_{1}-6 \pi / n, s_{1}+\right.$ $6 \pi / n ; 0,2 \pi ; \ldots ; 0,2 \pi] \cup \cdots \cup\left[0,2 \pi ; \ldots ; 0,2 \pi ; s_{l}-6 \pi / n, s_{l}+6 \pi / n\right] ; \Delta_{0}^{*}=D \backslash \Delta_{0} ;$ $\Delta_{k_{1}, \ldots, k_{l}}=\left[t_{k_{1}}, t_{k_{1}+1} ; \ldots ; t_{k_{l}}, t_{k_{l}+1}\right], k_{i}=0,1, \ldots, n-1, i=1,2, \ldots, l ; \Delta_{k_{1}, \ldots, k_{l}}^{*}=\Delta_{0}^{*} \cap \Delta_{k_{1}, \ldots, k_{l}}$, $k_{i}=0,1, \ldots, n-1, i=1,2, \ldots, l$, is asymptotically optimal among all quadrature rules as (4.17) and has the error

$$
R_{n \cdots n}\left[H_{1}^{\alpha}\right]=(1+o(1)) 2^{l} \pi^{\alpha-l} \frac{\ln ^{l} N l^{l-1}}{(l+\alpha) N^{\alpha / l}} .
$$

Consider the quadrature rule

$$
\begin{aligned}
H f=\frac{1}{4 \pi^{2}} \sum_{k=1}^{n-1} \sum_{i=1}^{n-1}[ & f\left(t_{k}, \tau_{i}\right) \iint_{g_{k i}^{*}} \operatorname{ctg} \frac{\sigma_{1}-s_{1}}{2} \operatorname{ctg} \frac{\sigma_{2}-s_{2}}{2} d \sigma_{1} d \sigma_{2} \\
& \left.+f\left(t_{k}^{\prime}, \tau_{i}^{\prime}\right) \iint_{d_{k i}^{*}} \operatorname{ctg} \frac{\sigma_{1}-s_{1}}{2} \operatorname{ctg} \frac{\sigma_{2}-s_{2}}{2} d \sigma_{1} d \sigma_{2}\right]+R_{n n},
\end{aligned}
$$

where $\left(t_{k}, \tau_{i}\right)=(2 \pi k / n, 2 \pi i / n),\left(t_{k}^{\prime}, \tau_{i}^{\prime}\right)=((2 k+1) \pi / n,(2 i+1) \pi / n) ; \Delta_{0}=\left[s_{1}-\right.$ $\left.2 \pi / n, s_{1}+2 \pi / n ; 0,2 \pi\right] \cup\left[0,2 \pi ; s_{2}-2 \pi / n, s_{2}+2 \pi / n\right] ; g_{k i}$ and $d_{k i}$ are domains which are defined by the expressions $\left|s_{1}-t_{k}\right|+\left|s_{2}-\tau_{i}\right| \leq \pi / n$ and $\left|s_{1}-t_{k}^{\prime}\right|+\left|s_{2}-\tau_{i}^{\prime}\right| \leq \pi / n$, $g_{k i}^{*}=\left([0,2 \pi]^{2} \backslash \Delta_{0}\right) \cap g_{k i}, d_{k i}^{*}=\left([0,2 \pi]^{2} \backslash \Delta_{0}\right) \cap d_{k i}, k, i=0,1, \ldots, n-1$.

TheOrem 4.16 (see $[6,8])$. Let $N=2 n^{2}, l=2$. Let $\Psi=H_{2}^{\alpha}(0<\alpha \leq 1)$. The quadrature rule (4.30) is asymptotically optimal among all quadrature rules as (4.17) and has the error equal to

$$
R_{n n}\left[H_{2}^{\alpha}\right] \sim 2^{1+\alpha / 2} \pi^{\alpha-2} \frac{\ln ^{2} N}{(2+\alpha) N^{\alpha / 2}} .
$$

Theorem 4.17 (see $[6,8]$ ). Let $N=n^{2}, l=2,3, \ldots$ Let $\Psi=H_{j}^{\alpha}, j=1,3,0<\alpha \leq 1$. Let the integral $G f$ be calculated with quadrature rule of the type (4.18), where $\rho_{i}=0$, $i=1,2$. Then

$$
\begin{aligned}
& \zeta_{N}\left[H_{1}^{\alpha}\right] \geq(1+o(1)) \frac{2^{l-\alpha}}{N^{\alpha / 2}(1+\alpha)} \ln ^{2} N, \\
& \zeta_{N}\left[H_{3}^{\alpha}\right] \geq 4(1+o(1)) N \ln ^{2} N \int_{0}^{1 / 2 n} \int_{0}^{1 / 2 n}\left(t^{2}+\tau^{2}\right)^{\alpha / 2} d t d \tau .
\end{aligned}
$$

TheOREM 4.18 (see [8]). Let $\Psi=H_{j}^{\alpha}, j=1,2,3,0<\alpha \leq 1$. Let the integral $G f$ be calculated with quadrature rule of the type (4.19). Let $l=2$ if $j=2,3$. Then $\zeta_{N}\left[H_{j}^{\alpha}\right] \geq$ $(1+o(1)) 2^{l} D_{j} N^{-\alpha / l} \ln ^{l} N$, where $D_{1}, D_{2}, D_{3}$ are defined in Theorem 4.8. 
Consider a quadrature rule

$$
G f=\sum_{k_{1}=1}^{n-1} \cdots \sum_{k_{l}}^{n-1} f\left(t_{k_{1}}^{\prime}, \ldots, t_{k_{l}}^{\prime}\right) \int \cdots \int_{d_{k_{1}, \ldots, k_{l}}} \frac{d \tau_{1} \cdots d \tau_{l}}{\left(\tau_{1}-t_{1}\right) \cdots\left(\tau_{l}-t_{l}\right)}+R_{N},
$$

where $d_{k_{1}, \ldots, k_{l}}=\Delta_{k_{1}, \ldots, k_{l}} \cap \Delta_{0}^{*}, \Delta_{0}^{*}=[0,1]^{l} \backslash \Delta_{0}, \Delta_{0}=\left[t_{1}-1 / n, t_{1}+1 / n ;-1,1 ; \ldots ;-1,1\right]$ $\cup \cdots \cup\left[-1,1 ; \ldots ;-1,1 ; t_{l}-1 / n, t_{l}+1 / n\right] ; t_{k}=k / n, k=0,1, \ldots, n ; t_{k}^{\prime}=\left(t_{k}+t_{k+1}\right) / 2$, $k=0,1, \ldots, n-1$.

TheOrem 4.19 (see $[6,8]$ ). Let $\Psi=H_{j}^{\alpha}, j=1,3,0<\alpha \leq 1$. Let $1 / n \leq t \leq 1-1 / n$, $i=1,2, \ldots, l$. Let $l=2$ if $j=3$. Among all quadrature rules of the type (4.18) provided $\rho_{i}=0, i=1, \ldots, l$, the quadrature rule (4.33) is asymptotically optimal and has the error

$$
\begin{aligned}
& R_{N}\left[H_{1}^{\alpha}\right]=(1+o(1)) 2^{l-\alpha} N^{-\alpha / l} l(1+\alpha)^{-1} \ln ^{l} N, \\
& R_{N}\left[H_{3}^{\alpha}\right]=4(1+o(1)) N^{2} \ln ^{2} N \int_{0}^{1 / 2 n} \int_{0}^{1 / 2 n}\left(t^{2}+t_{1}^{2}\right)^{\alpha / 2} d t d t_{1} .
\end{aligned}
$$

We consider a quadrature rule

$$
\begin{aligned}
G f=\sum_{k_{1}=0}^{n-1} \sum_{k_{2}=0}^{n-1}[ & f\left(v_{k_{1}}, w_{k_{2}}\right) \int_{a_{k_{1} k_{2}}^{*}} \frac{d \tau_{1} d \tau_{2}}{\left(\tau_{1}-t_{1}\right)\left(\tau_{2}-t_{2}\right)} \\
& \left.+f\left(v_{k_{1}}^{\prime}, w_{k_{2}}^{\prime}\right) \int_{d_{k_{1} k_{2}}^{*}} \frac{d \tau_{1} d \tau_{2}}{\left(\tau_{1}-t_{1}\right)\left(\tau_{2}-t_{2}\right)}\right]+R_{n n},
\end{aligned}
$$

where $\left(v_{k}, w_{l}\right)=(k / n, l / n),\left(v_{k}^{\prime}, w_{l}^{\prime}\right)=(k / n+1 / 2 n, l / n+1 / 2 n), k, l=0,1, \ldots, n ; \Delta_{0}=$ $\left[t_{1}-1 / n, t_{1}+1 / n ; 0,1\right] \cup\left[0,1 ; t_{2}-1 / n, t_{2}+1 / n\right] ; q_{k l}$ and $d_{k l}$ are domains which are defined by the expressions $\left|t_{1}-v_{k}\right|+\left|t_{2}-w_{l}\right| \leq 1 / 2 n$ and $\left|t_{1}-v_{k}^{\prime}\right|+\left|t_{2}-w_{l}^{\prime}\right| \leq 1 / 2 n$; $\Delta_{0}^{*}=[0,1]^{2} \backslash \Delta_{0} ; q_{k l}^{*}=q_{k l} \cap \Delta_{0}^{*}, d_{k l}^{*}=d_{k l} \cap \Delta_{0}^{*}, k, l=0,1, \ldots, n-1$.

TheOrem 4.20 (see $[7,8])$. Let $N=2 n^{2}$. Let $\Psi=H_{2}^{\alpha}(0<\alpha \leq 1)$. Among all quadrature rules of the type (4.19) the quadrature rule (4.35) is asymptotically optimal.

TheOrem 4.21 (see $[7,8]$ ). Let $\Psi=H_{j}^{\alpha}, j=1,3,0<\alpha \leq 1$. Let the integral $K f$ be calculated with quadrature rule of the type (4.20) provided $\rho_{1}=\rho_{2}=0$. The estimates occur

$$
\begin{aligned}
& \zeta_{N}\left[H_{1}^{\alpha}\right] \geq \frac{(1+o(1))}{2^{\alpha}(1+\alpha)} N^{-\alpha / 2} \ln N \int_{0}^{2 \pi}|f(\cos \phi, \sin \phi)| d \phi \\
& \zeta_{N}\left[H_{3}^{\alpha}\right] \geq(1+o(1)) 4 N^{-\alpha / 2} \ln N \int_{0}^{2 \pi}|f(\cos \phi, \sin \phi)| d \phi .
\end{aligned}
$$

THEOREM 4.22 (see $[7,8]$ ). Let $\Psi=H_{j}^{\alpha}, j=1,2,3$, and the integral $K \phi$ be evaluated with cubature rule as (4.21) ( $l=2$ if $j=2,3$ ). The estimate

$$
\zeta_{N}\left[H_{j}^{\alpha}\right] \geq \frac{1}{2}(1+o(1)) D_{j} N^{-\alpha / 2} \ln N \int_{0}^{2 \pi}|f(\cos \phi, \sin \phi)| d \phi
$$

is valid. The constants $D_{j}$ are defined in Theorem 4.8 . 
THEOREM 4.23 (see $[7,8]$ ). Let $\Psi=H_{j}^{\alpha}, j=1,3, l=2$. Among the cubature rules as (4.20) for $\rho_{1}=\rho_{2}=0$ the formula

$$
\begin{aligned}
K \phi= & \sum_{k=0}^{n-1} \sum_{m=0}^{n-1} \phi\left(t_{k}^{\prime}, t_{m}^{\prime}\right) \iint_{\Delta_{k m}} \frac{f(\theta) d \tau_{1} d \tau_{2}}{\left(\tau_{1}-t_{1}\right)^{2}+\left(\tau_{2}-t_{2}\right)^{2}} \\
& +\phi\left(t_{i}^{\prime}, t_{j}^{\prime}\right) \int_{\left[t_{i-1}, t_{i+2} ; t_{j-1}, t_{j+2}\right]} \int \frac{f(\theta) d \tau_{1} d \tau_{2}}{\left(\tau_{1}-t_{1}\right)^{2}+\left(\tau_{2}-t_{2}\right)^{2}}+R_{N}
\end{aligned}
$$

is asymptotically optimal for $1 / n \leq t_{i} \leq 1-1 / n, i=1,2$. Here $\left(t_{1}, t_{2}\right) \in \Delta_{i j}$, the prime in the summation indicate that $(k, m) \neq(i-1, j-1),(i, j-1), \ldots,(i+1, j+1)$. The cubature formula error is equal to

$$
\begin{aligned}
& R_{N}\left[H_{1}^{\alpha}\right]=\frac{1+o(1)}{2^{\alpha}(1+\alpha)} N^{-\alpha / 2} \ln N \int_{0}^{2 \pi}|f(\cos \phi, \sin \phi)| d \phi, \\
& R_{N}\left[H_{3}^{\alpha}\right]=(1+o(1)) 4 N^{-\alpha / 2} \ln N \int_{0}^{2 \pi}|f(\cos \phi, \sin \phi)| d \phi .
\end{aligned}
$$

Consider a formula

$$
\begin{aligned}
K \phi=\sum_{k_{1}=0}^{n-1} \sum_{k_{2}=0}^{n-1}(\phi & \left(v_{k_{1}}, w_{k_{2}}\right) \iint_{q_{k_{1} k_{2}}^{*}} \frac{f(\theta) d \tau_{1} d \tau_{2}}{\left(\tau_{1}-t_{1}\right)^{2}+\left(\tau_{2}-t_{2}\right)^{2}} \\
& \left.+\phi\left(v_{k_{1}}^{\prime}, w_{k_{2}}^{\prime}\right) \iint_{d_{k_{1} k_{2}}^{*}} \frac{f(\theta) d \tau_{1} d \tau_{2}}{\left(\tau_{1}-t_{1}\right)^{2}+\left(\boldsymbol{\tau}_{2}-t_{2}\right)^{2}}\right)+R_{N},
\end{aligned}
$$

where the definitions of formula (4.35) are used.

Theorem 4.24 (see $[7,8]$ ). Let $N=2 n^{2}, \Psi=H_{j}^{\alpha}, j=1,2$. Among all possible cubature rules as (4.21) the formula (4.40) is asymptotically optimal on class $\Psi$ for $2 / n \leq t_{i} \leq 1-2 / n, i=1,2$. The error is equal to

$$
R_{N}\left[H_{j}^{\alpha}\right]=\frac{1}{2}(1+o(1)) D_{j} N^{-\alpha / 2} \ln N \int_{0}^{2 \pi}|f(\cos \phi, \sin \phi)| d \phi .
$$

Proof OF TheOrem 4.11. For simplicity we assume that $l=2, n_{1}=n_{2}=n$. We introduce nodes $v_{k}=2 k \pi / M, k=0,1,2, \ldots, M, M=[n / \ln n]$. The union of the nodes $\left(x_{k_{1}}, x_{k_{2}}\right), 1 \leq x_{k_{1}}, x_{k_{2}} \leq n$ of quadrature rule (4.16) and the nodes $\left(v_{k}, v_{l}\right), k, l=$ $1,2, \ldots, M$, we denote by $\left(w_{k_{1}}, w_{k_{2}}\right), k_{1}, k_{2}=1,2, \ldots, L, L \leq n+M$. For each node $\left(v_{i}, v_{j}\right)$, $i, j=1,2, \ldots, M$, we compare the function

$$
\psi_{i j}^{*}\left(\sigma_{1}, \sigma_{2}\right)=\left\{\begin{aligned}
& 0, \quad \text { if }\left(\sigma_{1}, \sigma_{2}\right) \in \cup_{k=0}^{1}\left[v_{i-a}+k \pi, v_{i+a}+k \pi ; 0,2 \pi\right] \\
& \times \cup_{l=0}^{1}\left[0,2 \pi ; v_{j-a}+l \pi, v_{j+a}+l \pi\right], \\
& \psi\left(\sigma_{1}, \sigma_{2}\right) \operatorname{sgn}(\left.\operatorname{ctg} \frac{\sigma_{1}-v_{i}}{2} \operatorname{ctg} \frac{\sigma_{2}-v_{j}}{2}\right) \\
& \text { in other points of the domain } D,
\end{aligned}\right.
$$

where $a=\left[2^{1 / \alpha}\right]+1, \psi\left(\sigma_{1}, \sigma_{2}\right)=\left(\min _{i}\left|\sigma_{1}-w_{i}\right|^{\alpha}\right)+\left(\min _{j}\left|\sigma_{2}-w_{j}\right|^{\alpha}\right)$ is the function which was introduced in [34, 35]. 
It is easy to see that

$$
\begin{aligned}
&\left(F \psi_{i j}^{*}\right)\left(v_{i}, v_{j}\right) \\
&=\frac{1}{(2 \pi)^{2}} \int_{0}^{2 \pi} \int_{0}^{2 \pi} \psi_{i j}^{*}\left(\sigma_{1}, \sigma_{2}\right) \operatorname{ctg} \frac{\sigma_{1}-v_{i}}{2} \operatorname{ctg} \frac{\sigma_{2}-v_{j}}{2} d \sigma_{1} d \sigma_{2} \\
& \geq \sum_{k=a}^{[M / 2]-1} \sum_{l=a}^{[M / 2]-1} \frac{1}{(2 \pi)^{2}}\left[\operatorname{ctg} \frac{\pi(k+1)}{M} \operatorname{ctg} \frac{\pi(l+1)}{M} \int_{v_{k+i}}^{v_{k+i+1}} \int_{v_{l+j}}^{v_{l+j+1}} \psi\left(\sigma_{1}, \sigma_{2}\right) d \sigma_{1} d \sigma_{2}\right. \\
&+\operatorname{ctg} \frac{\pi(k+1)}{M} \operatorname{ctg} \frac{\pi(l+2)}{M} \int_{v_{k+i}}^{v_{k+i+1}} \int_{v_{j-l-1}}^{v_{j-l}} \psi\left(\sigma_{1}, \sigma_{2}\right) d \sigma_{1} d \sigma_{2} \\
&+\operatorname{ctg} \frac{\pi(k+2)}{M} \operatorname{ctg} \frac{\pi(l+1)}{M} \int_{v_{i-k-1}}^{v_{i-k}} \int_{v_{j+l}}^{v_{j+l+1}} \psi\left(\sigma_{1}, \sigma_{2}\right) d \sigma_{1} d \sigma_{2} \\
&\left.+\operatorname{ctg} \frac{\pi(k+2)}{M} \operatorname{ctg} \frac{\pi(l+2)}{M} \int_{v_{i-k-1}}^{v_{i-k}} \int_{v_{j-l-1}}^{v_{j-l}} \psi\left(\sigma_{1}, \sigma_{2}\right) d \sigma_{1} d \sigma_{2}\right] .
\end{aligned}
$$

Averaging this inequality on $i$ and $j$, we have

$$
\begin{aligned}
& \sup _{\varphi \in H_{\alpha \alpha}} \max _{s_{1} s_{2}}(F \varphi)\left(s_{1}, s_{2}\right) \geq \frac{1}{M^{2}} \sum_{i=0}^{M-1} \sum_{j=0}^{M-1}\left(F \psi_{i j}^{*}\right)\left(v_{i}, v_{j}\right) \\
& \geq \frac{1}{\pi^{2}} \int_{0}^{2 \pi} \int_{0}^{2 \pi} \psi\left(\sigma_{1}, \sigma_{2}\right) d \sigma_{1} d \sigma_{2} \frac{1}{M^{2}} \sum_{k=a}^{[M / 2]-1} \sum_{j=a}^{[M / 2]-1} \operatorname{ctg} \frac{\pi(k+2)}{M} \operatorname{ctg} \frac{\pi(j+2)}{M} \\
& =\frac{1+o(1)}{\pi^{4}} \ln ^{2} n \int_{0}^{2 \pi} \int_{0}^{2 \pi} \psi\left(\sigma_{1}, \sigma_{2}\right) d \sigma_{1} d \sigma_{2} .
\end{aligned}
$$

So,

$$
\sup _{\varphi \in H_{\alpha \alpha}} \max _{s_{1} s_{2}}(F \varphi)\left(s_{1}, s_{2}\right) \geq(1+o(1)) 8 \pi^{\alpha-2}(1+\alpha)^{-1} n^{-\alpha} \ln ^{2} n
$$

This proves Theorem 4.11 .

Other theorems of this section are proved by similar way but technically more complicated.

4.3. Asymptotically optimal methods of the calculation of the polysingular integrals on Sobolev classes of functions. Consider the polysingular integrals as

$$
F f=\int_{0}^{1} \int_{0}^{1} f\left(\sigma_{1}, \sigma_{2}\right) \operatorname{ctg} \pi\left(\sigma_{1}-s_{1}\right) \operatorname{ctg} \pi\left(\sigma_{2}-s_{2}\right) d \sigma_{1} d \sigma_{2} .
$$

For evaluating the integral $F f$ we use the following quadrature rules

$$
F f=\sum_{k=1}^{m} \sum_{l=1}^{n} \sum_{i=0}^{\rho_{1}} \sum_{j=0}^{\rho_{2}} p_{k l, i j}\left(s_{1}, s_{2}\right) f^{(i, j)}\left(x_{k}, y_{l}\right)+R_{n m}\left(s_{1}, s_{2} ; p_{k l, i j} ; x_{k}, y_{l} ; f\right) \text {. }
$$


TheOrem 4.25 (see $[6,8])$. Let $\Psi=\tilde{C}_{2}^{r}(1), r=1,2, \ldots$ Let the integral (4.46) be calculated with quadrature rules of the type (4.47), where $\rho_{1}+\rho_{2} \leq r, n=m$. Then

$$
\zeta_{n n}[\Psi] \geq \frac{(4+o(1)) R_{r 1}(1) \ln ^{2} n}{(r+1) !(2 n)^{r} \pi^{2}} .
$$

TheOREM 4.26 (see $[6,8]$ ). Let $\Psi=\tilde{W}^{r s}(1), r, s=1,2, \ldots$ Let the integral (4.46) be calculated with quadrature rules of the type (4.47), where $\rho_{1}=r-1, \rho_{2}=s-1$. Then

$$
\zeta_{m n}[\Psi] \geq \frac{4+o(1)}{\pi^{2}}\left(\frac{R_{r 1}(1)}{(2 m)^{r}(r+1) !}+\frac{R_{s 1}(1)}{(2 n)^{s}(s+1) !}\right) \ln n \ln m .
$$

Construct the special polynomial for approximating function $f\left(t_{1}, t_{2}\right)$ belonging to the class $W^{r s}(1)$ on the rectangle $[a, b ; c, d]$. Let $t_{1}$ be fixed value. Introduce a polynomial

$$
f_{S}\left(t_{1}, t_{2}\right)=\sum_{l=0}^{s-1}\left[\frac{f^{(0, l)}\left(t_{1}, c\right)}{l !}\left(t_{2}-c\right)^{l}+B_{l} \delta^{(l)}\left(t_{1}, d\right)\right]
$$

where

$$
\delta\left(t_{1}, t_{2}\right)=f\left(t_{1}, t_{2}\right)-\sum_{l=0}^{s-1} \frac{f^{(0, l)}\left(t_{1}, c\right)}{l !}\left(t_{2}-c\right)^{l} .
$$

The coefficients $B_{l}$ are defined from the equality

$$
\left(d-t_{2}\right)^{s}-\sum_{l=0}^{s-1} \frac{B_{l}(d-c) s !}{(s-l-1) !}\left(d-t_{2}\right)^{s-l-1}=(-1)^{s} R_{s 1}\left(\frac{c+d}{2}, \frac{d-c}{2}, t_{2}\right),
$$

where $R_{s p}(a, h, t)$ is the polynomial of order $s$ with respect to the variable $t$ deviating least from zero in the space $L_{p}[a-h, a+h]$.

Special polynomial $f_{r s}\left(t_{1}, t_{2} ;[a, b ; c, d]\right)$ is defined by the formula

$$
f_{r s}\left(t_{1}, t_{2} ;[a, b ; c, d]\right)=\sum_{l=0}^{r-1}\left[\frac{f_{s}^{(l, 0)}\left(a, t_{2}\right)}{l !}\left(t_{1}-a\right)^{l}+B_{l} \delta^{(l)}\left(b, t_{2}\right)\right],
$$

where

$$
\delta\left(t_{1}, t_{2}\right)=f_{s}\left(t_{1}, t_{2}\right)-\sum_{l=0}^{r-1} \frac{f_{s}^{(l, 0)}\left(a, t_{2}\right)}{l !}\left(t_{1}-a\right)^{l} .
$$

The coefficients $B_{l}$ are defined from the equality

$$
\left(b-t_{1}\right)^{r}-\sum_{l=0}^{r-1} \frac{B_{l}(b-a) r !}{(r-l-1) !}\left(b-t_{1}\right)^{r-l-1}=(-1)^{r} R_{r 1}\left(\frac{a+b}{2}, \frac{b-a}{2}, t_{1}\right) .
$$

Cover the square $D=[0,1]^{2}$ with parallelepipeds $\Delta_{k l}=\left[t_{k}, t_{k+1} ; \tau_{l}, \tau_{l+1}\right], k=0,1, \ldots$, $n-1, l=0,1, \ldots, m-1$, where $t_{k}=k / n, k=0,1, \ldots, n, \tau_{l}=l / m, l=0,1, \ldots, m$. Assume that the singular integral (4.46) is calculated at the point $\left(s_{1}, s_{2}\right)$. Let $\left(s_{1}, s_{2}\right) \in \Delta_{i, j}$. We approximate the function $f\left(s_{1}, s_{2}\right)$ by the function $f_{r s}\left(s_{1}, s_{2} ; \Delta_{k l}\right)$ in the parallelepiped $\Delta_{k l}$ for $(k, l)$ so that $k \neq i-1, i, i+1$ or $l \neq j-1, j, j+1$. In the parallelepiped $\Delta_{k l}$ for $k=i-1, i, i+1, l=0,1, \ldots, m-1$ or $k=0,1, \ldots, n-1, l=j-1, j, j+1$ function $f\left(\sigma_{1}, \sigma_{2}\right)$ 
is approximated by Taylor series $T_{r-1, s-1}\left(f, \Delta_{k l},\left(t_{k}, \tau_{l}\right)\right)$. Local spline is constructed in $[0,1]^{2}$ thus we define as $\tilde{f}_{n, m}\left(\sigma_{1}, \sigma_{2}\right)$.

Consider the quadrature rule

$$
F f=\int_{0}^{1} \int_{0}^{1} \tilde{f}_{r s}\left(\sigma_{1}, \sigma_{2}\right) \operatorname{ctg} \pi\left(\sigma_{1}-s_{1}\right) \operatorname{ctg} \pi\left(\sigma_{2}-s_{2}\right) d \sigma_{1} d \sigma_{2}+R_{n m}\left(s_{1}, s_{2}\right) .
$$

THEOREM 4.27 (see $[6,8]$ ). Let $\Psi=\tilde{W}^{r, s}(1)$. Among all quadrature rules of the type (4.47) the quadrature rule (4.56) is asymptotically optimal and has the error

$$
R_{n m}[\Psi]=(1+o(1)) 4 \ln n \ln m\left(\frac{R_{r 1}(1)}{\pi^{2}(r+1) !(2 n)^{r}}+\frac{R_{s 1}(1)}{\pi^{2}(s+1) !(2 m)^{s}}\right) .
$$

Consider a quadrature rule

$$
G f=\int_{0}^{1} \int_{0}^{1} \frac{f\left(\tau_{1}, \tau_{2}\right)}{\left(\tau_{1}-t_{1}\right)\left(\tau_{2}-t_{2}\right)} d \tau_{1} d \tau_{2}=\sum_{k_{1}=0}^{n-1} \sum_{k_{2}=0}^{n-1} \iint_{\Delta_{k_{1} k_{2}}} \frac{f_{n n}\left(\tau, \Delta_{k_{1} k_{2}}\right)}{\left(\tau_{1}-t_{1}\right)\left(\tau_{2}-t_{2}\right)} d \tau_{1} d \tau_{2}+R_{n},
$$

where $\tau=\left(\tau_{1}, \tau_{2}\right) ; \Delta_{i j}=\left[t_{i}, t_{i+1} ; t_{j}, t_{j+1}\right], t_{i}=i / n, i=0,1, \ldots, n$.

THEOREM 4.28 (see [7, 8]). Let $N=n^{2}$ and $\Psi=W^{r, r}(1)$. Among all possible cubature rules as (4.18) for $l=2, \rho_{i}=r-1, i=1,2$, the formula (2.64) is asymptotically optimal. Its estimation is $R_{N}[\Psi]=(1+o(1)) 2 \ln ^{2} N / r ! 4^{r} N^{r / 2}$.

THEOREM 4.29 (see $[7,8]$ ). Let $\Psi=W^{r, r}(1)$ and the integral $K \phi$ be evaluated with cubature rule as (4.20) for $l=2, \rho_{i}=r-1, i=1,2$. It is valid the estimation

$$
\zeta_{N}[\Psi] \geq \frac{(1+o(1)) \ln N}{r ! 4^{r} N^{r / 2}} \int_{0}^{2 \pi}|f(\cos \phi, \sin \phi)| d \phi .
$$

THEOREM 4.30 (see [7, 8]). Let $N=n^{2}$ and $\Psi=W^{r, r}(1)$. Among all possible cubature rules as (4.20) for $l=2, \rho_{i}=r-1, i=1,2 ; 2 / n \leq t_{i} \leq 1-2 / n, i=1,2$; the formula

$$
K \phi=\sum_{k=0}^{n-1} \sum_{i=0}^{n-1} \int_{\Delta_{k i}} \int \frac{\phi_{n n}\left(\tau_{1}, \tau_{2}\right) f(\theta) d \tau_{1} d \tau_{2}}{\left(\tau_{1}-t_{1}\right)^{2}+\left(\tau_{2}-t_{2}\right)^{2}}+R_{N}
$$

where we use as designations as in (4.58), is asymptotically optimal on class $\Psi$. Its error is equal to

$$
R_{N}[\Psi]=\frac{(1+o(1)) \ln N}{r ! 4^{r} N^{r / 2}} \int_{0}^{2 \pi}|f(\cos \phi, \sin \phi)| d \phi .
$$

Proofs of these theorems are given in $[7,8]$.

4.4. Optimal with respect to order quadrature rules on Hölder classes. Consider polysingular integrals as (4.13) for calculation of which we shall use the quadrature rules (4.16) and (4.17). For simplicity we shall put $l=2$.

Let $D=[0,2 \pi]^{2}$. We cover the square $D$ with parallelepipeds $\Delta_{k l}=\left[t_{k}, t_{k+1} ; \tau_{l}, \tau_{l+1}\right]$, $k=0,1, \ldots, n-1, l=0,1, \ldots, m-1$, where $t_{k}=2 k \pi / n, \tau_{l}=2 l \pi / m, k=0,1, \ldots, n$, $l=0,1, \ldots, m$. 
Theorem 4.31 (see $[6,8])$. On classes $H_{\alpha_{1}, \alpha_{2}}(1), H_{1}^{\alpha}(D)$ and $H_{3}^{\alpha}(D)\left(0<\alpha, \alpha_{1}\right.$, $\left.\alpha_{2} \leq 1\right)$ the quadrature rule

$$
H f=\frac{1}{4 \pi^{2}} \sum_{k=0}^{n-1} \sum_{l=0}^{m-1} f\left(t_{k}^{\prime}, \tau_{l}^{\prime}\right) \iint_{\Delta_{k l}} \operatorname{ctg} \frac{\sigma_{1}-t_{i}^{\prime}}{2} \operatorname{ctg} \frac{\sigma_{2}-\tau_{j}^{\prime}}{2} d \sigma_{1} d \sigma_{2}+R_{n m}\left(s_{1}, s_{2} ; f\right),
$$

where $\left(s_{1}, s_{2}\right) \in \Delta_{i j} ; t_{k}^{\prime}=(2 k+1) \pi / n, k=0,1, \ldots, n-1 ; \tau_{l}^{\prime}=(2 l+1) \pi / m, l=0,1, \ldots$, $m-1$; is optimal with respect to order. The error holds

$$
\begin{gathered}
R_{n m}\left[H_{\alpha_{1}, \alpha_{2}}(1)\right] \asymp 4 \ln \frac{n}{2} \ln \frac{m}{2}\left(\frac{2^{1+\alpha_{1}}-1}{\left(1+\alpha_{1}\right) \pi^{2-\alpha_{1}} n^{\alpha_{1}}}+\frac{2^{1+\alpha_{2}}-1}{\left(1+\alpha_{2}\right) \pi^{2-\alpha_{2}} m^{\alpha_{2}}}\right), \\
R_{n m}\left[H_{3}^{\alpha}(D)\right] \asymp \frac{4 n m}{\pi^{4}}(\ln n+o(\ln n))(\ln m+o(\ln m)) \int_{0}^{2 \pi / n} \int_{0}^{2 \pi / m}\left(s_{1}^{2}+s_{2}^{2}\right)^{\alpha / 2} d s_{1} d s_{2}, \\
R_{n m}\left[H_{1}^{\alpha}(D)\right] \asymp 2^{2+\alpha} \pi^{\alpha-2} n^{-\alpha} \ln ^{2} n+8 e \pi^{\alpha-2} \alpha^{-1} n^{-\alpha} \ln n .
\end{gathered}
$$

5. Quadrature rules for Hadamard finite part integrals. In this section, we investigate the approximate methods of calculation of one-dimensional and many-dimensional Hadamard finite-part integrals.

5.1. Introduction. Suppose that a function $f(t)$ integrable over $[a, b]$ belongs to the class $W^{r}(M), r \geq p, p=1,2, \ldots$. The Hadamard finite part (f.p.) integral is given by

$$
\int_{a}^{b} \frac{f(t) d t}{(b-t)^{p+\alpha}}=\lim _{x \rightarrow b}\left[\int_{a}^{x} \frac{f(t) d t}{(b-t)^{p+\alpha}}+\frac{B(x)}{(b-x)^{p+\alpha-1}}\right]
$$

where $0<\alpha<1$. The function $B(x)$ is an arbitrary function which satisfies the following conditions:

(1) limit in (5.1) exists.

(2) the function $B(x)$ has at least the derivatives of order $p$ in a neighbourhood of the point $b$.

Sampling the function $B(x)$ does not influence the values of the Hadamard f.p. integral. The Cauchy-Hadamard finite-part integral is given by

$$
\int_{a}^{b} \frac{f(t) d t}{(t-c)^{p}}=\lim _{\eta \rightarrow 0}\left[\int_{a}^{c-\eta} \frac{f(t) d t}{(t-c)^{p}}+\int_{c+\eta}^{b} \frac{f(t) d t}{(t-c)^{p}}+\frac{B(\eta)}{(c-\eta)^{p-1}}\right]
$$

where $p=2,3, \ldots, a<c<b$. The function $B(x)$ is chosen thus that the limit in (5.2) exists.

5.2. Asymptotically optimal quadrature rules for the calculation of the Hadamard finite part integrals with fixed singularity. In this section, we review the quadrature rules for the computation of the Hadamard f.p. integrals

$$
\begin{aligned}
& I f=\int_{-1}^{1} \frac{f(t) d t}{t^{v}}, \quad v=2,3, \ldots, \\
& L f=\int_{-1}^{1} \frac{f(t) d t}{|t|^{v+\alpha}}, \quad v=1,2, \ldots, 0<\alpha<1 .
\end{aligned}
$$


We will use the following quadrature rules:

$$
\begin{aligned}
& I f=\sum_{k=-N}^{N} \sum_{l=0}^{\rho} p_{k l} f^{(l)}\left(t_{k}\right)+R_{N}\left(p_{k l}, t_{k}, f\right), \\
& L f=\sum_{k=-N}^{N} \sum_{l=0}^{\rho} p_{k l} f^{(l)}\left(t_{k}\right)+R_{N}\left(p_{k l}, t_{k}, f\right) .
\end{aligned}
$$

We will use the local spline $\tilde{f}\left(t, \Delta_{k}\right)$ that was constructed in the section preliminaries of the introduction.

THEOREM 5.1 (see [11]). Set $\Psi=W_{p}^{r}(1), 1 \leq p \leq \infty$. Among all possible quadrature rules of the type (5.5), where $\rho=r-1$, the formula

$$
\begin{aligned}
I f= & \sum_{k=0}^{r-1} \frac{f^{(k)}(0)}{k !(k+1-v)} t_{1}^{k+1-v}\left(1-(-1)^{r+1-v}\right) \\
& +\sum_{k=-N, k \neq-1,0}^{N-1} \int_{t_{k}}^{t_{k+1}} \frac{\tilde{f}\left(t,\left[t_{k}, t_{k+1}\right]\right) d t}{t^{v}}+R_{N},
\end{aligned}
$$

where $t_{k}= \pm(k / N)^{(r+1 / q)(r+1 / q-v)}, 1 / p+1 / q=1$ is asymptotically optimal. The error occurs

$$
R_{N}[\Psi]=\frac{(1+o(1)) R_{r q}(1)}{2^{r-1 / q}(r q+1)^{1 / q} r !}\left(\frac{r+1 / q}{r+1 / q-v}\right)^{r+1 / q} \frac{1}{N^{r}} .
$$

TheOrem 5.2 (see [11]). Set $\Psi=W_{p}^{r}(1), r=1,2, \ldots, 1 \leq p \leq \infty$. Among all possible quadrature rules of the type (5.6) providing $\rho=r-1$, the formula

$$
\begin{aligned}
L f= & \sum_{k=v+1}^{r-1} \frac{2 f^{(k)}(0)}{k !(k+1-v-\alpha)} t_{1}^{k+1-v-\alpha} \\
& +\sum_{k=-N, k \neq-1,0}^{N-1} \int_{t_{k}}^{t_{k+1}} \frac{\tilde{f}\left(t,\left[t_{k}, t_{k+1}\right]\right) d t}{|t|^{v+\alpha}}+R_{N},
\end{aligned}
$$

where $t_{ \pm k}= \pm(k / N)^{(r+1 / q)(r+1 / q-v-\alpha)}$ is asymptotically optimal. The error holds

$$
R_{N}\left[W_{p}^{r}(1)\right]=\frac{(1+o(1)) R_{r q}(1)}{2^{r-1 / q}(r q+1)^{1 / q} r ! N^{r}}\left(\frac{r+1 / q}{r+1 / q-v-\alpha}\right)^{r+1 / q}
$$

Proofs of Theorems 5.1 and 5.2 are similar to the proof of Theorem 2.7.

5.3. Evaluation of the Hadamard finite part integrals on finite curves. Let $L$ be an arbitrary piece-continuous closed curve. Let $f(t) \in W^{r}(1)$. In this section we investigate quadrature rules for the Hadamard f.p. integrals of the following type

$$
A f=\int_{L} \frac{f(\tau) d \tau}{(\tau-t)^{v}}
$$

Let $t_{k}, k=0,1, \ldots, N$ are the equidistant points on the closed curve $L$. Let $\bar{t}_{k}$ be the equidistant point from $t_{k}$ and $t_{k+1}, k=0,1, \ldots, N-1$. 
Let $t_{0}=t_{N}$. Consider a quadrature rule

$$
A f=\frac{1}{2} \sum_{k=0}^{N-1} \bar{f}\left(\bar{t}_{k}\right) \int_{t_{k}}^{t_{k+1}}\left(\frac{1}{(\tau-t+\bar{n} h)^{v}}+\frac{1}{(\tau-t-\bar{n} h)^{v}}\right) d \tau+R_{N}
$$

where $\bar{n}$ is outside the normal vector to the curve $L$ at the point $\bar{t}_{k}, h=0\left(N^{-1 / v}\right)$.

We assume that the values $f(t)$ at the points $\bar{t}_{k}$ can be computed up to $\epsilon: \mid \bar{f}\left(\bar{t}_{k}\right)-$ $f\left(\bar{t}_{k}\right) \mid \leq \epsilon$.

THEOREM 5.3 (see [11]). Let $f \in W^{r}(1), r \geq v$. The quadrature rule (5.12) error $R_{N}=A\left(N^{-1 / v} \ln N+\epsilon N^{1-1 / v}\right)$ is valid.

Consider the Hadamard f.p. integrals

$$
H f=\int_{-1}^{1} \frac{f(\tau) d \tau}{(\tau-t)^{v}}
$$

Let $t_{k}=-1+2 k / N, k=0,1, \ldots, N, t_{k}^{\prime}=\left(t_{k}+t_{k+1}\right) / 2, k=0,1, \ldots, N-1$. Let $h=N^{-1 / v}$. We assume that the singular point $t$ belongs to the segment $[-1+\delta, 1-\delta], \delta \geq h$. We also assume that $t \in\left[t_{j}, t_{j+1}\right)$. We suppose that the values $f\left(t_{k}^{\prime}\right)$ can be calculated up to $\epsilon:\left|\bar{f}\left(t_{k}^{\prime}\right)-f\left(t_{k}^{\prime}\right)\right| \leq \epsilon$.

We will compute the integrals as (5.13) with the quadrature rule

$$
\begin{aligned}
H f= & \sum_{k=0, k \neq j-1, j, j+1}^{N-1} \overline{f\left(t_{k}^{\prime}\right)} \int_{t_{k}}^{t_{k+1}}\left(\frac{1}{(\tau-(t-i h))^{v}}+\frac{1}{(\tau-(t+i h))^{v}}\right) d \tau \\
& +\overline{f\left(t_{j}^{\prime}\right)} \int_{t_{j-1}}^{t_{j+2}}\left(\frac{1}{(\tau-(t-i h))^{v}}+\frac{1}{(\tau-(t+i h))^{v}}\right) d \tau+R_{N} .
\end{aligned}
$$

THEOREM 5.4 (see [11]). Let $f \in W^{r}(1), r \geq v$. The quadrature rule (5.14) error $R_{N}=A\left(N^{-1 / v} \ln N+\epsilon N^{1-1 / v}\right)$ is valid.

Application of the interpolated polynomial to the value of the Hadamard integrals is illustrated on the example of the integral

$$
A_{1} f=\int_{-1}^{1} \frac{f(\tau) d \tau}{\left(1-\tau^{2}\right)^{1 / 2}(\tau-t)^{2}} .
$$

Approximate the function $f(t)$ by the interpolated polynomial

$$
f_{n}(t)=L_{n}(f)=\sum_{k=0}^{n}\left(\frac{1}{\gamma_{k}} \sum_{i=0}^{n} T_{i}\left(\mu_{k}\right) T_{i}(t)\right) f\left(\mu_{k}\right)
$$

where $T_{m}(t)=\sqrt{2 / \pi} \cos (m \arccos t)$ are Chebyshev polynomials of type I of degree $m ; \mu_{k}=\cos ((2 k-1) \pi) /(2 n+2), k=1,2, \ldots, n+1$ are the nodes of the polynomial $T_{n+1}(t) ; \gamma_{k}=\sum_{l=0}^{n} T_{l}^{2}\left(\mu_{k}\right)$. 
Substituting $f_{n}(t)$ instead of $f(t)$ into the integral $A_{1} f$ and having taken advantage of the formulae [28]

$$
\int_{-1}^{1} \frac{T_{n}(\tau) d \tau}{\left(1-\tau^{2}\right)^{1 / 2}(\tau-t)^{2}}=0
$$

for $n=0,1$ and

$$
\int_{-1}^{1} \frac{T_{n}(\tau) d \tau}{\left(1-\tau^{2}\right)^{1 / 2}(\tau-t)^{2}}=\frac{\pi}{1-t^{2}}\left(-\frac{n-1}{2} U_{n}(t)+\frac{n+2}{2} U_{n-2}(t)\right)
$$

for $n=2, \ldots$, we obtain the quadrature rule

$$
\begin{aligned}
\int_{-1}^{1} & \frac{f(\tau) d \tau}{\left(1-\tau^{2}\right)^{1 / 2}(\tau-t)^{2}} \\
& =\frac{\pi}{1-t^{2}} \sum_{k=0}^{n}\left[\frac{f\left(\mu_{k}\right)}{\gamma_{k}} \sum_{i=2}^{n} T_{i}\left(\mu_{k}\right)\left(-\frac{i-1}{2} U_{i}(t)+\frac{i+1}{2} U_{i-2}(t)\right)\right]+R_{n},
\end{aligned}
$$

where $U_{n}(t)$ are Chebyshev polynomials of the type II.

THEOREM 5.5 (see [14]). The quadrature rule (5.19) error $\left|R_{n}\right| \leq A E_{n}(f) n^{2} \lambda_{n}$ is valid.

Proof. Estimate value of the error of the quadrature rule (5.19)

$$
\left|R_{n}\right|=\left|\int_{-1}^{1} \frac{\left(f(\tau)-f_{n}(\tau)\right) d \tau}{\left(1-\tau^{2}\right)^{1 / 2}(\tau-t)^{2}}\right|=\left|\int_{-1}^{1} \frac{\psi_{n}(\tau) d \tau}{\left(1-\tau^{2}\right)^{1 / 2}(\tau-t)^{2}}\right|,
$$

where $\psi_{n}(\tau)=f(\tau)-f_{n}(\tau)$. Having made use of the Taylor formula with the remainder term in integral form we obtain $\psi_{n}(\tau)=\psi_{n}(t)+\left(\psi_{n}^{\prime}(t) / 1\right.$ ! $)(\tau-t)+(1 / 1$ !) $\int_{t}^{\tau}(\tau-v) \psi_{n}^{\prime \prime}(v) d v$. So,

$$
\begin{aligned}
\left|R_{n}\right|= & \left|\int_{-1}^{1} \frac{\psi_{n}(\tau) d \tau}{\left(1-\tau^{2}\right)^{1 / 2}(\tau-t)^{2}}\right| \\
= & \left|\int_{-1}^{1} \frac{\psi_{n}(t)+\psi_{n}^{\prime}(t)(\tau-t)+\int_{t}^{\tau}(\tau-v) \psi_{n}^{\prime \prime}(v) d v}{\left(1-\tau^{2}\right)^{1 / 2}(\tau-t)^{2}} d \tau\right| \\
\leq & \max _{t}\left|\psi_{n}(t) \int_{-1}^{1} \frac{d \tau}{\left(1-\tau^{2}\right)^{1 / 2}(\tau-t)^{2}}\right|+\max _{t}\left|\psi_{n}^{\prime}(t) \int_{-1}^{1} \frac{d \tau}{\left(1-\tau^{2}\right)^{1 / 2}(\tau-t)}\right| \\
& +\max _{t}\left|\psi_{n}^{\prime \prime}(t)\right| \frac{1}{2} \int_{-1}^{1} \frac{d \tau}{\left(1-\tau^{2}\right)^{1 / 2}} .
\end{aligned}
$$

It is known [28] that $\int_{-1}^{1} d \tau /\left(1-\tau^{2}\right)^{1 / 2}(\tau-t)^{2}=0, \int_{-1}^{1} d \tau /\left(1-\tau^{2}\right)^{1 / 2}(\tau-t)=0$, $-1<t<1$.

Therefore,

$$
\left|R_{n}\right| \leq \frac{\pi}{2} \max _{t}\left|\psi_{n}^{\prime \prime}(t)\right| .
$$


The interpolation error by the polynomial $f_{n}(t)$ occurs $\left|\psi_{n}(t)\right|=\left|f(t)-f_{n}(t)\right| \leq$ $E_{n}(f)\left(1+\lambda_{n}\right)$. Using Markov inequality [34, 35] and the circuit of the Bernstein theorem proof about structural properties of functionals [34,35], it is possible to show that

$$
\left|\psi_{n}^{\prime \prime}(t)\right| \leq A n^{2} E_{n}(f)\left(1+\lambda_{n}\right) .
$$

It is known [34, 35] that for the points of Chebyshev polynomials of the type I

$$
\lambda_{n} \leq A \ln n
$$

Collecting the estimations (5.21), (5.22), (5.23), and (5.24) we finish the proof of the theorem.

Similar results are obtained [14] for different weight functions and for different integer degrees of the difference $(\tau-t)$.

5.4. Weight quadrature rules for the Hadamard finite part integrals. Consider the Hadamard f.p. integral

$$
H f=\int_{0}^{1} \frac{\rho(\tau) f(\tau)}{(\tau-t)^{v}} d \tau, \quad v=2,3, \ldots
$$

For simplicity we assume that $\rho(t)=t^{-\gamma}, 0 \leq \gamma<1$. We use the interpolated polynomials $P_{r}\left(f ;\left[s_{k}, s_{k+1}\right]\right)$ for approximation of the function $f(t)$ on the segment $\left[s_{k}, s_{k+1}\right]$. The construction of this polynomials was described in Section 1.3.

Let $f \in W^{r}(1), r>p-1$. Let $s_{k}=(k / N)^{q}, q=r /(r-\gamma), k=0,1, \ldots, N$. Let $t \in$ $\left[s_{j}, s_{j+1}\right]$. The Hadamard f.p. integrals of the type (5.25) we evaluate with the quadrature rule

$$
\begin{aligned}
H f= & \sum_{k=0}^{j-2} \int_{s_{k}}^{s_{k+1}} \frac{P_{r}\left(f ;\left[s_{k}, s_{k+1}\right]\right)}{\tau^{\gamma}(\tau-t)^{v}} d \tau+\sum_{k=j+2}^{N-1} \int_{s_{k}}^{s_{k+1}} \frac{P_{r}\left(f ;\left[s_{k}, s_{k+1}\right]\right)}{\tau^{\gamma}(\tau-t)^{v}} d \tau \\
& +\int_{s_{j-1}}^{s_{j+2}} \frac{P_{r}\left(f ;\left[s_{j-1}, s_{j+2}\right]\right)}{\tau^{\gamma}(\tau-t)^{v}} d \tau+R_{N} .
\end{aligned}
$$

THEOREM 5.6 (see [11]). Let $\Psi=W^{r}(1)$. Among all possible quadrature rules of the type $H f=\sum_{k=1}^{N} \sum_{l=0}^{\rho} p_{k l}(t) f^{(l)}\left(t_{k}\right)+R_{N}\left(t, p_{k l}, t_{k}, f\right)$ with $0 \leq \rho \leq r$ the quadrature rule (5.26) is optimal with respect to order. The error of quadrature rule (5.26) $\left|R_{N}\right| \leq$ $A N^{-r(r+1-v-\gamma) /(r-\gamma)}$ for $t \in\left[s_{2}, s_{N-2}\right]$ is valid.

5.5. Evaluation of the many-dimensional Hadamard finite part integrals. Consider the following type of Hadamard f.p. integrals

$$
I f=\int_{L_{1}} \int_{L_{2}} \frac{f\left(\tau_{1}, \tau_{2}\right) d \tau_{1} d \tau_{2}}{\left(\tau_{1}-t_{1}\right)^{p_{1}}\left(\tau_{2}-t_{2}\right)^{p_{2}}},
$$

where $L_{i}, i=1,2$ are piece-continuously closed curves.

Divide the closed curve $L_{1}$ into $N_{1}$ equal parts by nodes $t_{k_{1}}, k_{1}=0,1, \ldots, N_{1}, t_{0}=$ $t_{N_{1}}$. Divide the closed curve $L_{2}$ into $N_{2}$ equal parts by the nodes $t_{k_{2}}, k_{2}=0,1, \ldots, N_{2}$, $t_{0}=t_{N_{2}}$. Let $\bar{n}_{i}, i=1,2$ be a unit normal to the curve $L_{i}, i=1,2$. 
We will compute the integral (5.27) with the quadrature rule

$$
\begin{aligned}
& I f=\frac{1}{4} \sum_{k_{1}=0}^{N_{1}-1} \sum_{k_{2}=0}^{N_{2}-1} f\left(t_{k_{1}}^{\prime}, t_{k_{2}}^{\prime}\right) \\
& \times \int_{t_{k_{1}}}^{t_{k_{1}+1}} \int_{t_{k_{2}}}^{t_{k_{2}+1}}\left[\frac{1}{\left(\tau_{1}-\left(t_{1}+\bar{n}_{1} h_{1}\right)\right)^{p_{1}}\left(\tau_{2}-\left(t_{2}+\bar{n}_{2} h_{2}\right)\right)^{p_{2}}}\right. \\
&+\frac{1}{\left(\tau_{1}-\left(t_{1}+\bar{n}_{1} h_{1}\right)\right)^{p_{1}}\left(\tau_{2}-\left(t_{2}-\bar{n}_{2} h_{2}\right)\right)^{p_{2}}} \\
&+\frac{1}{\left(\tau_{1}-\left(t_{1}-\bar{n}_{1} h_{1}\right)\right)^{p_{1}}\left(\tau_{2}-\left(t_{2}+\bar{n}_{2} h_{2}\right)\right)^{p_{2}}} \\
&+\left.\frac{1}{\left(\tau_{1}-\left(t_{1}-\bar{n}_{1} h_{1}\right)\right)^{p_{1}}\left(\tau_{2}-\left(t_{2}-\bar{n}_{2} h_{2}\right)\right)^{p_{2}}}\right] d \tau_{1} d \tau_{2}+R_{N_{1} N_{2}},
\end{aligned}
$$

where $t_{k_{i}}^{\prime}, i=1,2$ is the point equidistant from the points $t_{k_{i}}$ and $t_{k_{i}+1}, i=1,2, h_{i}=$ $N_{i}^{-1 / p_{i}}, i=1,2$.

THEOREM 5.7 (see [11]). Let $\Psi=\tilde{W}^{r_{1} r_{2}}(1), r_{i}>1, i=1$, 2. Let $p_{1}=p_{2}=2$. Let $h_{i}=$ $N_{i}^{-1 / 2}, i=1,2$. The quadrature rule (5.28) error is equal to $\left|R_{N_{1} N_{2}}\right| \leq A h_{1} h_{2}\left|\ln h_{1} \ln h_{2}\right|$.

Consider the Hadamard f.p. integrals of the following type

$$
A f=\int_{-1}^{1} \int_{-1}^{1} \frac{f\left(\tau_{1}, \tau_{2}\right)}{\left(\tau_{1}-t_{1}\right)^{p_{1}}\left(\tau_{2}-t_{2}\right)^{p_{2}}} d \tau_{1} d \tau_{2} .
$$

For the evaluation of the integrals of the type (5.29) we introduce the following quadrature rule

$$
\begin{aligned}
& A f=\frac{1}{4} \sum_{k_{1}=0}^{N_{1}-1} \sum_{k_{2}=0}^{N_{2}-1} f\left(t_{k_{1}}^{\prime}, t_{k_{2}}^{\prime}\right) \\
& \times \int_{t_{k_{1}}}^{t_{k_{1}+1}} \int_{t_{k_{2}}}^{t_{k_{2}+1}}\left(\frac{1}{\left(\tau_{1}-\left(t_{1}-i h_{1}\right)\right)^{p_{1}}\left(\tau_{2}-\left(t_{2}-i h_{2}\right)\right)^{p_{2}}}\right. \\
&+\frac{1}{\left(\tau_{1}-\left(t_{1}+i h_{1}\right)\right)^{p_{1}}\left(\tau_{2}-\left(t_{2}-i h_{2}\right)\right)^{p_{2}}} \\
&+\frac{1}{\left(\tau_{1}-\left(t_{1}-i h_{1}\right)\right)^{p_{1}}\left(\tau_{2}-\left(t_{2}+i h_{2}\right)\right)^{p_{2}}} \\
&\left.+\frac{1}{\left(\tau_{1}-\left(t_{1}+i h_{1}\right)\right)^{p_{1}}\left(\tau_{2}-\left(t_{2}+i h_{2}\right)\right)^{p_{2}}}\right) d \tau_{1} d \tau_{2}+R_{N_{1} N_{2}},
\end{aligned}
$$

where $t_{k_{i}}=-1+2 k_{i} / N_{i}\left(k_{i}=0,1, \ldots, N_{i}\right) ; t_{k}^{\prime}=\left(t_{k}+t_{k+1}\right) / 2, h_{i}=N_{i}^{-1 / p_{i}}, i=1,2$.

TheOREM 5.8 (see [11]). Let $\Psi=W^{r_{1} r_{2}}(1)$. Let $p_{1}=p_{2}=p, h_{1}=h_{2}=h, r_{1}=r_{2}=r$. The quadrature rule (5.30) error $\left|R_{N_{1} N_{2}}\right| \leq A h^{2}\left|\ln ^{2} h\right|$ is valid. 
Consider the Hadamard f.p. integrals of the following type

$$
A f=\int_{-\infty}^{\infty} \int_{-\infty}^{\infty} \frac{f\left(\tau_{1}, \tau_{2}\right) d \tau_{1} d \tau_{2}}{\left(\tau_{1}-t_{1}\right)^{2}\left(\tau_{2}-t_{2}\right)^{2}} .
$$

We assume that the function $f\left(t_{1}, t_{2}\right)$ can be represented as $f\left(t_{1}, t_{2}\right)=\rho_{i}\left(t_{i}, t_{2}\right)$ $g\left(t_{1}, t_{2}\right)$, where $\rho_{i}\left(t_{1}, t_{2}\right), i=1,2$, is a weight function, $g\left(t_{1}, t_{2}\right)$ is a smooth function.

As weight functions we will use the following functions $\rho_{1}\left(t_{1}, t_{2}\right)=a^{-\left|t_{1}\right|-\left|t_{2}\right|}$, $a>1 ; \rho_{2}\left(t_{1}, t_{2}\right)=e^{-t_{1}^{2}-t_{2}^{2}}$.

DEFINITION 5.9. Class $W^{r_{1} r_{2}}(1, k), r_{i}=1,2, \ldots, i=1,2$, consists of the functions $f\left(t_{1}, t_{2}\right)$ which are defined on domain $(-\infty, \infty)^{2}$. These functions have the continuous derivatives $f^{(1,0)}\left(t_{1}, t_{2}\right), f^{(0,1)}\left(t_{1}, t_{2}\right), \ldots, f^{\left(r_{1}, r_{2}-1\right)}\left(t_{1}, t_{2}\right), f^{\left(r_{1}-1, r_{2}\right)}\left(t_{1}, t_{2}\right)$ and the piece-continuous derivative $f^{\left(r_{1}, r_{2}\right)}\left(t_{1}, t_{2}\right)$. Functions $f\left(t_{1}, t_{2}\right)$ and its derivatives satisfy the following conditions: $\max \left|f^{\left(r_{1}, r_{2}\right)}\left(t_{1}, t_{2}\right)\right| \leq 1, \max \left(\left\|f\left(t_{1}, t_{2}\right)\right\|,\left\|f^{(1,0)}\left(t_{1}, t_{2}\right)\right\|\right.$, $\left.\left\|f^{(0,1)}\left(t_{1}, t_{2}\right)\right\|, \ldots,\left\|f^{\left(r_{1}, r_{2}-1\right)}\left(t_{1}, t_{2}\right)\right\|,\left\|f^{\left(r_{1}-1, r_{2}\right)}\left(t_{1}, t_{2}\right)\right\|\right) \leq k$.

Let $N$ be integer. Let $r_{1}=r_{2}=r$. Let $A_{1}=\left[r \log _{a} N\right], A_{2}=[\ln N]$, where $[a]$ is the greatest integer in $a$. Let $N_{k}^{1}=N / a^{|k| r}, k=-A_{1}, \ldots,-1,0,1, \ldots, A_{1}, N_{k}^{2}=N / \exp \left(k^{2} / r\right)$, $k=-A_{2}, \ldots,-1,0,1, \ldots, A_{2}$. Let $t_{k, l}^{1}=k+l / N_{k}^{1}, k=-A_{1}, \ldots,-1,0,1, \ldots, A_{1} ; l=0,1, \ldots, N_{k}^{1}$, $t_{k, l}^{2}=k+l / N_{k}^{2}, k=-A_{2}, \ldots,-1,0,1, \ldots, A_{2} ; l=0,1, \ldots, N_{k}^{2}$.

TheOREM 5.10 (see [11]). Let $\Psi=W^{(r, r)}(1, k), r>p-1$. Let $h=N^{-1 / p}$. The quadrature rule

$$
\begin{aligned}
& A\left(\rho_{i} g\right)=\frac{1}{4} \sum_{k_{1}=-A_{i}}^{A_{i}} \sum_{k_{2}=-A_{i}}^{A_{i}-1} \sum_{l_{1}=0}^{N_{k_{1}}^{i_{1}}} \sum_{l_{2}=0}^{N_{k_{2}}^{i_{1}}} \\
& \times \int_{t_{k_{1}, l_{1}}^{i}}^{t_{k_{1}, l_{1}+1}^{i}} \int_{t_{k_{2}, l_{2}}^{i}}^{t_{k_{2}, l_{2}+1}^{i}}\left(\frac{1}{\left(\tau_{1}-t_{1}+i h\right)^{2}\left(\tau_{2}-t_{2}+i h\right)^{2}}\right. \\
&+\frac{1}{\left(\tau_{1}-t_{1}+i h\right)^{2}\left(\tau_{2}-t_{2}-i h\right)^{2}} \\
&+\frac{1}{\left(\tau_{1}-t_{1}-i h\right)^{2}\left(\tau_{2}-t_{2}+i h\right)^{2}} \\
&\left.+\frac{1}{\left(\tau_{1}-t_{1}-i h\right)^{2}\left(\tau_{2}-t_{2}-i h\right)^{2}}\right) d \tau_{1} d \tau_{2}+R_{N_{1} N_{2}}
\end{aligned}
$$

has the error $\left|R_{N_{1} N_{2}}\right|=A h\left|\ln ^{2} h\right|+1 / N h^{2}$.

\section{REFERENCES}

[1] K. I. Babenko (ed.), Theoretical Bases and Construction of Numerical Algorithms for the Tasks of Mathematical Physics, Nauka, Moscow, 1979 (Russian).

[2] V. F. Babenko, An asymptotically accurate estimation of the remainder of best cubature formulas for certain classes of functions, Mat. Zametki 19 (1976), no. 3, 313322 (Russian), [translated in Math. Notes 19 (1976), 187-193. Zbl 333.41024]. MR 54\#5697. 
[3] N. S. Bakhvalov, O svoistvah optimalnih metodov resenia zadach matematicheskoi fiziki, Zh. Vychisl. Mat. Mat. Fiz. 10 (1970), no. 3, 555-588 (Russian), English translation: U.S.S.R. Computational Math. and Math. Phys. 10 (1970), no. 3, 1-10.

[4] , On the optimality of linear methods for operator approximation in convex classes of functions, Zh. Vychisl. Mat. Mat. Fiz. 11 (1971), no. 4, 244-249 (Russian), English translation: U.S.S.R. Comput. Math. and Math. Phys. 11 (1971), 244-249.

[5] I. V. Boikov, Optimal Methods of Calculation in Automatic Control Problems, Penz. Politekhn. Inst., Penza, 1983 (Russian).

[6] _ Optimal with Respect to Accuracy Algorithms of Approximate Calculation of Singular Integrals, Saratov State University Press, Saratov, 1983 (Russian).

[7] _ Asymptotically optimal cubature formulas for calculating multidimensional singular integrals, Trudy Inst. Vychisl. Mat. Akad. Nauk Gruzin. SSR 29 (1990), no. 1, 26-42 (Russian). MR 92j:41040.

[8] _ Passivnye i Adaptivnye Algoritmy Priblizhennogo Vychisleniya Singulyarnykh Integralov. Chast 2 [Passive and Adaptive Algorithms for the Approximate Computation of Singular Integrals. Part 2], Izdatelstvo Penzenskogo Gosudarstvennogo Tekhnicheskogo Universiteta, Penza, 1995 (Russian). MR 97f:65015.

[9] _ _ Passivnye i Adaptivnye Algoritmy Priblizhennogo Vychisleniya Singulyarnykh Integralov. Chast 1 [Passive and Adaptive Algorithms for the Approximate Computation of Singular Integrals. Part 1], Izdatelstvo Penzenskogo Gosudarstvennogo Tekhnicheskogo Universiteta, Penza, 1995 (Russian). MR 97f:65015.

[10] I. V. Boikov and A. N. Andreev, Optimal algorithms for the calculation of singular integrals, Integral Methods in Science and Engineering (Houghton, Michigan, 1998), Chapman Hall/CRC Res. Notes Math., vol. 418, Chapman \& Hall/CRC, Boca Raton, FL, 2000, pp. 67-72. CMP 1754 154. Zbl 957.65014.

[11] I. V. Boikov, N. F. Dobrunina, and L. N. Domnin, Approximate Methods of Calculation of Hadamard Integrals and Solution of Hypersingular Integral Equations, Penza Technical State Univ. Press, Penza, 1996 (Russian).

[12] I. V. Boikov and S. J. Nagaeva, Optimal with Respect to Order Algorithms for Calculation Singular and Hypersingular Integrals, vol. 26, Penza State University, Penza, 2001 (Russian), Paper is deposit in VINITI. 19.01.01. No. 150-B2001.

[13] I. V. Boikov and S. Y. Nagaeva, Optimal methods of calculation of singular integrals on axis, Optimalnie Methodi Vychislenii i Ix Primeneniy (Russian), vol. 12, Penza Technical State University Press, Penza, 1966, pp. 46-67 (Russian).

[14] A. Boikova, Methods for the approximate calculation of the Hadamard integral and solution of integral equations with Hadamard integrals, Integral Methods in Science and Engineering, Vol. 2 (Oulu, 1996), Pitman Res. Notes Math. Ser., vol. 375, Longman, Harlow, 1997, pp. 59-63. CMP 1611 460. Zbl 901.65092.

[15] M. M. Chawla and R. Kreß, Estimating errors of numerical approximation for periodic analytic functions, Computing 18 (1977), no. 3, 241-248. MR 56\#9915. Zbl 365.65016.

[16] M. M. Chawla and T. R. Ramakrishnan, Numerical evaluation of integrals of periodic functions with Cauchy and Poisson type kernels, Numer. Math. 22 (1974), 317-323. MR 51\#9437. Zbl 271.65018.

[17] G. Criscuolo and G. Mastroianni, On the convergence of an interpolatory product rule for evaluating Cauchy principal value integrals, Math. Comp. 48 (1987), no. 178, 725-735. MR 88m:65038. Zbl 638.65017.

[18] K. Diethelm, Gaussian quadrature formulae of the third kind for Cauchy principal value integrals: basic properties and error estimates, J. Comput. Appl. Math. 65 (1995), no. 1-3, 97-114. MR 96m:65024. Zbl 847.41019.

[19] J. Y. Du, Quadrature formulas for singular integrals with Hilbert kernel, J. Comput. Math. 6 (1988), no. 3, 205-225. MR 90b:41049. Zbl 668.65020. 
[20] D. Elliott and D. F. Paget, On the convergence of a quadrature rule for evaluating certain Cauchy principal value integrals, Numer. Math. 23 (1975), 311-319. MR 52\#1115. Zbl 313.65019.

[21] - On the convergence of a quadrature rule for evaluating certain Cauchy principal value integrals (Numer. Math. 23 (1975), 311-319): an addendum, Numer. Math. 25 (1976), no. 3, 287-289. MR 53\#14870. Zbl 321.65017.

[22] _ Gauss type quadrature rules for Cauchy principal value integrals, Math. Comp. 33 (1979), no. 145, 301-309. MR 81h:65023. Zbl 415.65019.

[23] D. Gaier, Konstruktive Methoden der Konformen Abbildung [Constructive Methods for Conformal Mapping], Springer Tracts in Natural Philosophy, vol. 3, Springer-Verlag, Berlin, 1964 (German). MR 33\#7507. Zbl 132.36702.

[24] D. B. Hunter, Some Gauss-type formulae for the evaluation of Cauchy principal values of integrals, Numer. Math. 19 (1972), 419-424. MR 47\#7899. Zbl 231.65028.

[25] N. I. Ioakimidis, Further convergence results for two quadrature rules for Cauchy type principal value integrals, Apl. Mat. 27 (1982), no. 6, 457-466. MR 84f:65017. Zbl 518.65009.

[26] V. V. Ivvanov, Optimal algorithms for the computation of singular integrals, Dokl. Akad. Nauk SSSR 204 (1972), 21-24 (Russian), [translated in Soviet Math. Dokl. 13 (1972), 576-580. Zbl 259.42017]. MR 47\#2819.

[27] _ The Theory of Approximate Methods and Their Application to the Numerical Solution of Singular Integral Equations, Monographs and Textbooks on Mechanics of Solids and Fluids, Mechanics: Analysis, vol. 2, Noordhoff International Publishing, Leyden, 1976. MR 53\#8841. Zbl 346.65065.

[28] A. C. Kaya and F. Erdogan, On the solution of integral equations with strongly singular kernels, Quart. Appl. Math. 45 (1987), no. 1, 105-122. MR 88e:45012. Zbl 631.65139.

[29] V. I. Lebedev and O. V. Baburin, On the computation of principal value integrals, the weights and nodes of Gaussian quadrature formulae, Ž. Vyčisl. Mat. i Mat. Fiz. 5 (1965), 454-462 (Russian), [translated in U.S.S.R. Comput. Math. and Math. Phys. 5 (1967), no. 3, 81-92. Zbl 156.17003]. MR 32\#6670.

[30] I. K. Lifanov, Metod Singulyarnykh Integral'nykh uravneniı̆ i chislennyĭ Ėksperiment $v$ Matematicheskol Fizike, Aèrodinamike, Teorii Uprugosti i Difraktsii Voln [The Method of Singular Integral Equations and a Numerical Experiment in Mathematical Physics, Aerodynamics and the Theory of Elasticity and Wave Diffraction], TOO "Yanus", Moscow, 1995 (Russian). MR 98h:45005. Zbl 904.73001.

[31] G. G. Lorentz, Approximation of Functions, 2nd ed., Chelsea Publishing Company, New York, 1986. MR 88j:41001. Zbl 643.41001.

[32] V. P. Motornii, On the Best Quadrature Rule of Type $\sum_{k=1}^{n} p_{k} f\left(t_{k}\right)$ for Some Classes of Periodic Functions, Izv. Akad. Nauk USSR, Ser. Matem. 38 (1974), no. 3, 583-614.

[33] B. I. Musaev, Quadraturformeln für ein singuläres Integral über einen Abschnitt der reellen Geraden [Quadrature formulas for a singular integral over an interval of the real line], Izv. Vysš. Učebn. Zaved. Matematika (1977), no. 8, 56-67 (Russian). MR 58\#8171. Zbl 387.65020.

[34] I. P. Natanson, Constructive Function Theory. Vol. II. Approximation in Mean, Frederick Ungar Publishing Co., New York, 1965. MR 33\#4529b. Zbl 136.36302.

[35] _ Constructive Function Theory. Vol. III. Interpolation and Approximation Quadratures, Frederick Ungar Publishing Co., New York, 1965. MR 33\#4529c. Zbl 178.39701.

[36] S. M. Nikol'skiĭ, Kvadraturnye Formuly [Quadrature Formulae], 3rd ed., Nauka, Moscow, 1979 (Russian). MR 81g:41034.

[37] M. G. Okun', Optimal quadrature formulas for the calculation of integrals with fixed weight, Optimal Computation Methods and Their Application, No. 7 (Russian), Penz. Politekhn. Inst., Penza, 1985, pp. 45-59, 162. CMP 00978893. Zbl 674.41017. 
[38] A. P. Prudnikov, Y. A. Brychkov, and O. I. Marichev, Integraly i ryady. Ehlementarnye funktsii [Integrals and Series. Elementary Functions], "Nauka”, Moscow, 1981 (Russian). MR 83b:00009. Zbl 511.00044.

[39] P. Rabinowitz and E. Santi, On the uniform convergence of Cauchy principal values of quasi-interpolating splines, BIT 35 (1995), no. 2, 277-290. MR 98b:65027. Zbl 859.65015.

[40] D. G. Sanikidze, On approximation Cauchy singular integrals and its limited values at the endpoints of integration curves, Math. Notes. 15 (1971), 533-542 (Russian).

[41] E. Santi, Uniform convergence results for certain two-dimensional Cauchy principal value integrals, Portugal. Math. 57 (2000), no. 2, 191-201. MR 2001a:65029. Zbl 0967.65035.

[42] M. S. Shabozov, An approach to the investigation of optimal quadrature formulas for singular integrals with a fixed singularity, Ukrain. Mat. Zh. 47 (1995), no. 9, 13001305 (Russian), [translation in Ukrainian Math. J. 47, no. 9, 1479-1485 (1996). Zbl 955.41023]. MR 98c:65035.

[43] S. L. Sobolev, Vvedenie v Teoriyu Kubaturnykh Formul[Introduction to Theory of Cubature Formulae], Izdat. Nauka, Moscow, 1974 (Russian). MR 57\#18037. Zbl 294.65013.

[44] F. Stenger, Sinc approximation of Cauchy-type integrals over arcs, ANZIAM J. 42 (2000), no. 1, 87-97. MR 2001h:30034. Zbl 01523466.

[45] A. I. Stepanets, Ravnomernye Priblizheniya Trigonometricheskimi Polinomami. Linĕnye Metody [Uniform Approximation by Trigonometrical Polynomials. Linear Methods], Naukova Dumka, Kiev, 1981 (Russian). MR 83k:42002. Zbl 481.42001.

[46] P. S. Theocaris and J. G. Kazantzakis, On the numerical evaluation of two-and threedimensional Cauchy principal-value integrals, Acta Mech. 39 (1981), no. 1-2, 105115. MR 82d:65029. Zbl 481.73076.

[47] J. F. Traub and H. Woźniakowsi, A General Theory of Optimal Algorithms, ACM Monograph Series, Academic Press, New York, 1980. MR 84m:68041. Zbl 441.68046.

I. V. Boikov: Penza State University, Krasnay Street, 40, PenZA, 440017, Russia

E-mail address: boikov@diamond.stup.ac.ru 


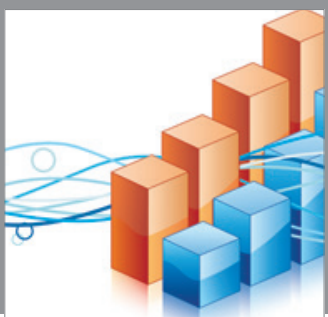

Advances in

Operations Research

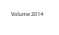

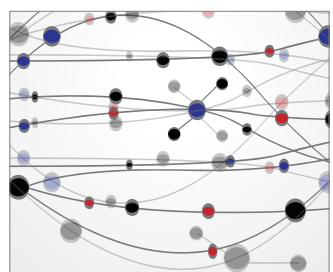

\section{The Scientific} World Journal
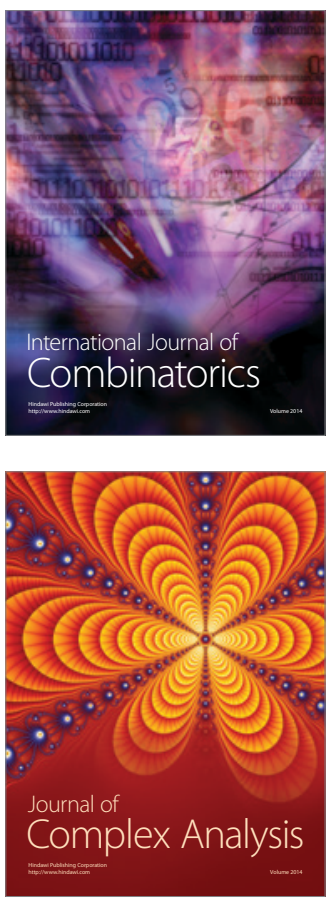

International Journal of

Mathematics and

Mathematical

Sciences
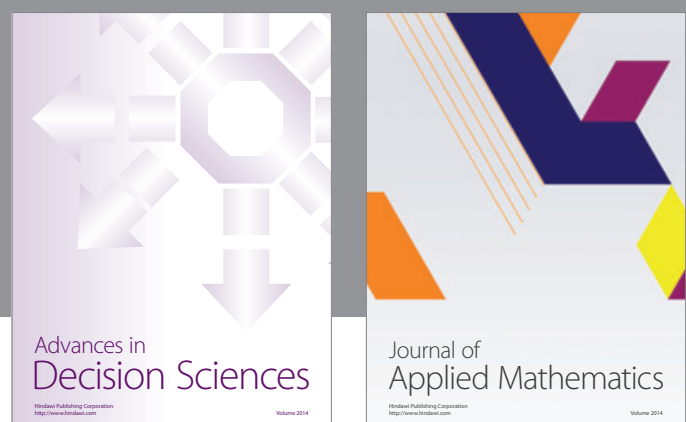

Journal of

Applied Mathematics
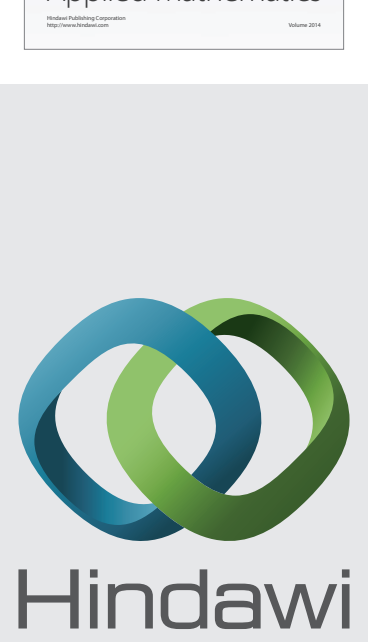

Submit your manuscripts at http://www.hindawi.com
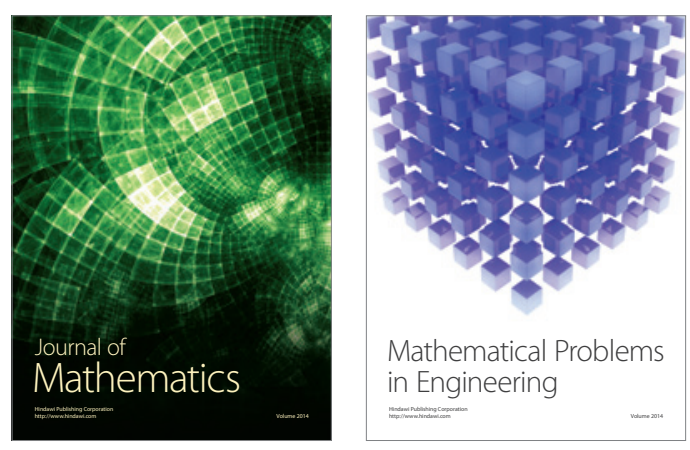

Mathematical Problems in Engineering
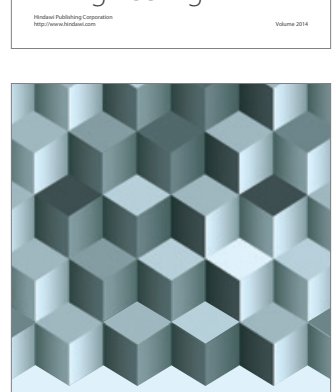

Journal of

Function Spaces
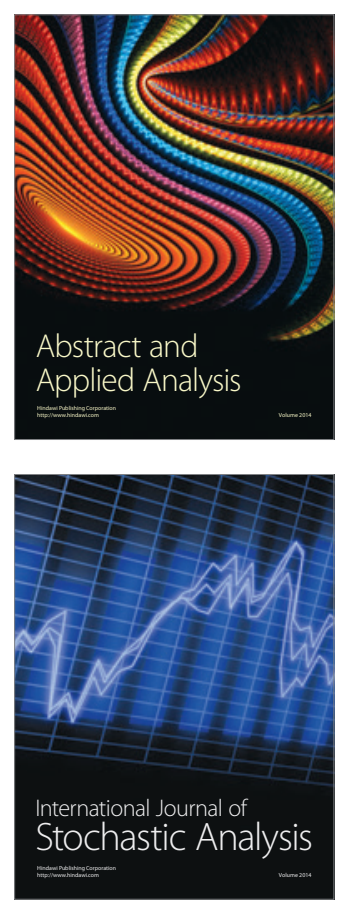

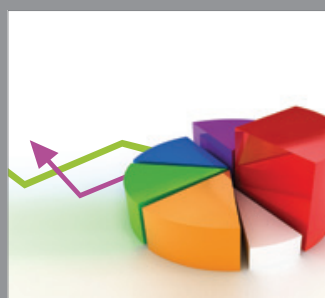

ournal of

Probability and Statistics

Promensencen
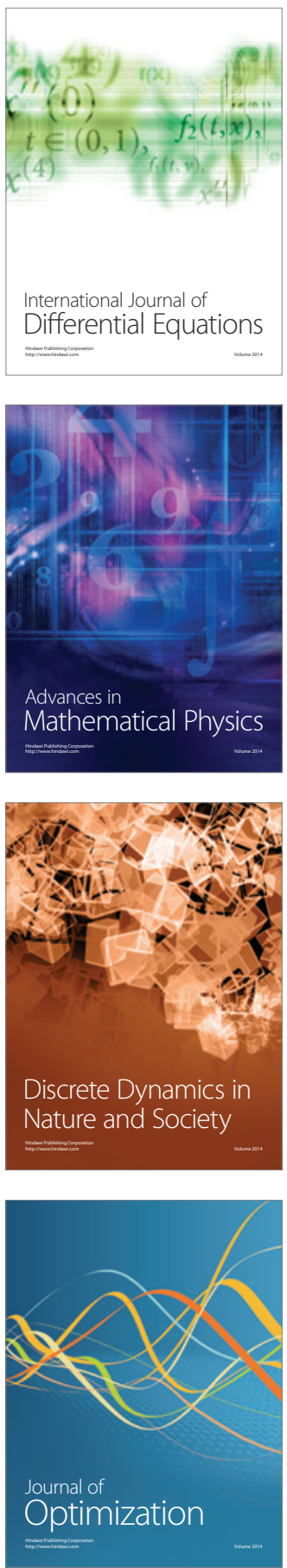